\title{
Mechanisms involved in type II macrophage activation and effector functions
}

\author{
By \\ Marie Clare Lydia Kharkrang \\ A thesis \\ submitted to the Victoria University of Wellington \\ in fulfilment of the requirements for the degree of \\ Master of Biomedical Science
}

Victoria University of Wellington

2010 


\section{Abstract}

Autoimmunities are extremely difficult to treat and involved in their pathogenesis are proinflammatory immune responses redirected against one's own tissues. Studies in our lab have shown macrophages that are induced to become type II macrophages protect against an animal model of MS, experimental autoimmune encephalomyelitis (EAE), with protection due to immune deviation. Another way to deviate immune responses away from inflammation is by infection with the parasitic helminth Schistosoma mansoni, which also protects against EAE. The contribution of type II macrophages in this protection is unknown, as are the mechanisms involved in promoting the phenotype induced by type II activation. This project investigates key mechanisms involved in type II activation, while also elucidating the possible effect of schistosome exposure on the induction of this activation state.

Using a validated model of type II activation in vitro, we compared the effects of schistosome immune complexes on various macrophage properties such as cytokine, surface marker and enzymatic profiles. This thesis identified that exposure to schistosome complexes induces a macrophage state with characteristics of two distinct activation states (type II and alternative activation), as well as completely novel characteristics. This activation state shows many phenotypic properties associated with immune regulation, and may have important consequences for understanding mechanisms involved in protection against inflammatory illnesses.

We also investigated key mechanisms involved in the anti-inflammatory responses induced by type II activation. Cytokine, chemokine and surface marker profiles of macrophages were assessed in response to type II activation in vitro, with the main emphasis on determining the effects of IL-10 and CD40 on the type II activation phenotype and function. This investigation found that type II activated macrophages depend on low levels of CD40/CD40L signalling to polarise Th2 development, as the expression of receptors for Th2-inducing cytokines are significantly impaired in the absence of this interaction. This suggests an important role for the low but maintained levels of CD40 on type II activated macrophages, in aiding the deviation of immune responses, while maintaining Th2 polarization. We also suggest a suppressive role of CD40/CD40L in IL-10 production, which is a novel find.

The requirement of new treatments for MS is escalating as more people are affected each year. The impact of MS on the quality of life is severe and long lasting. Having a greater understanding of the mechanisms involved in deviating pro-inflammatory or antiinflammatory responses will enable the development of much more effective treatments and therapies in the future. 


\section{Table of contents}

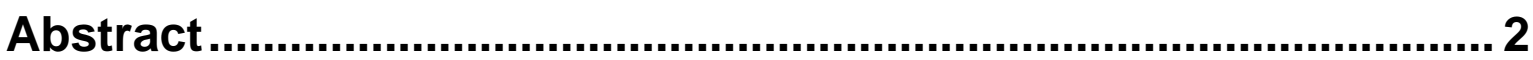

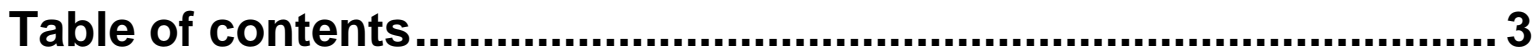

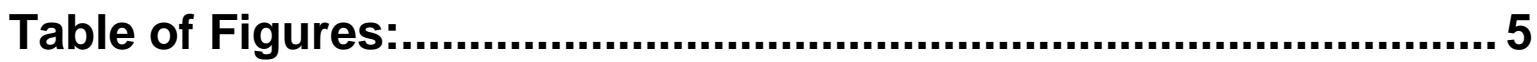

List of abbreviations: ..................................................................... 6

1.1 Innate and adaptive immune responses............................................... 10

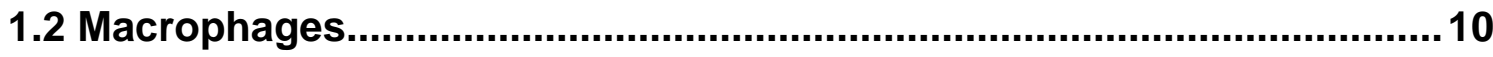

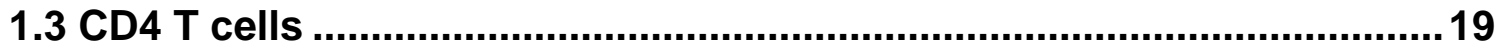

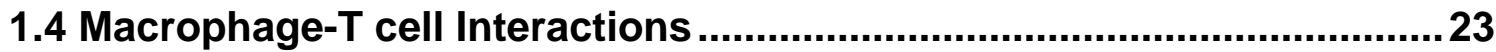

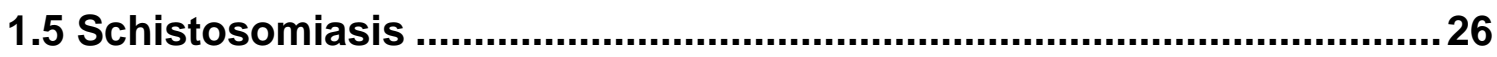

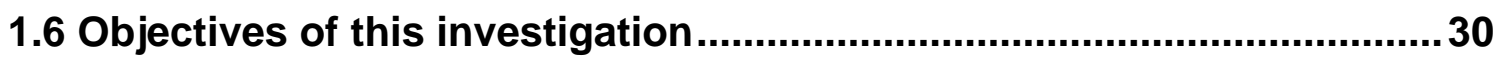

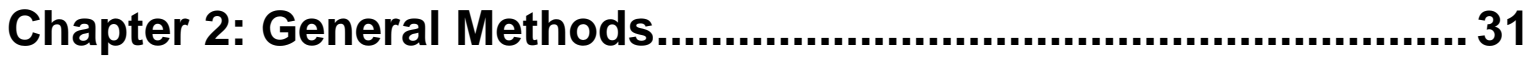

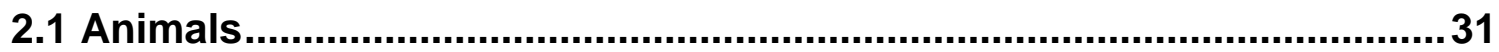

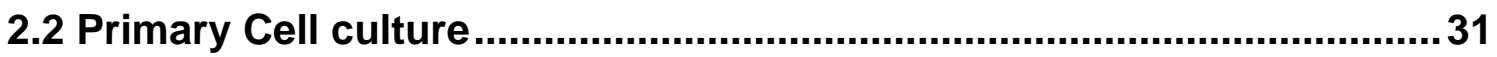

2.3 CFSE staining protocol of CD4 T cells. .................................................. 33

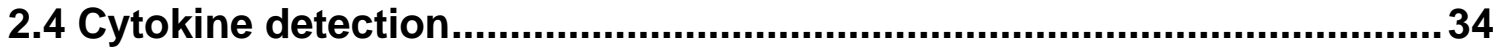

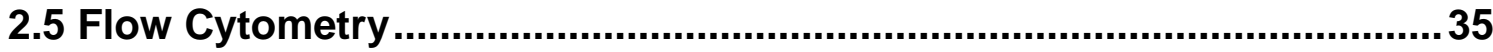

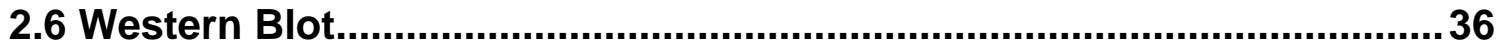

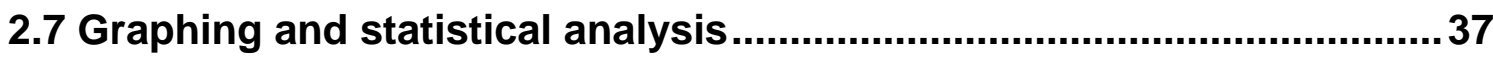

Chapter 3: Defining key mechanisms of type II macrophage

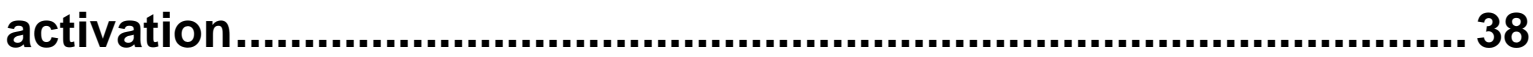

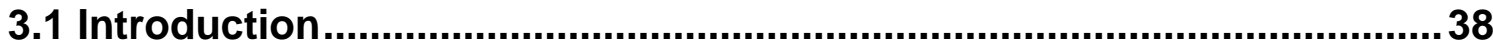

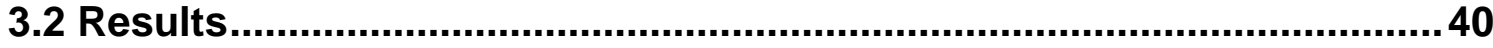

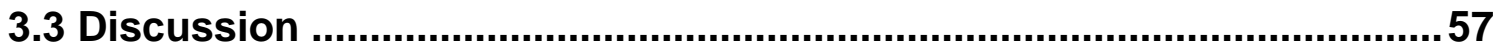

Chapter 4: Elucidating the effects of type II macrophages on $\mathrm{T}$

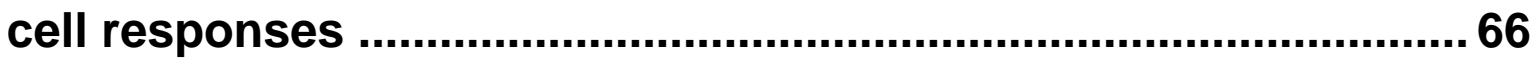

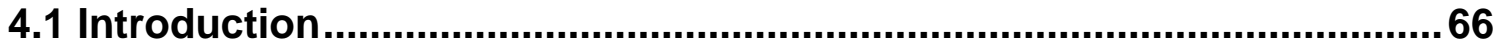

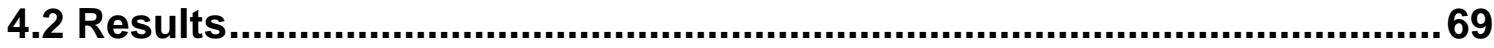

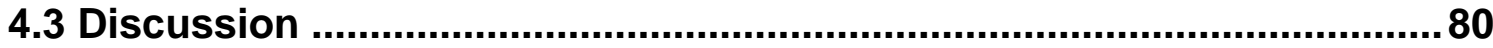

Chapter 5: Effects of schistosome complexes on macrophage

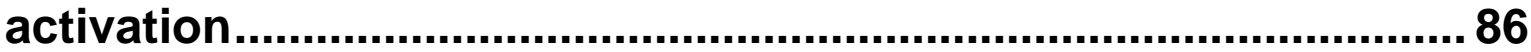

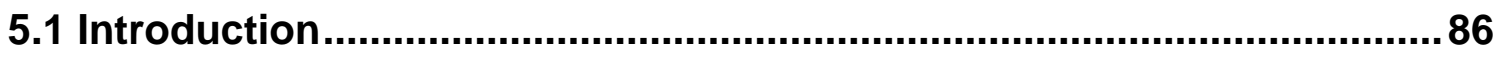

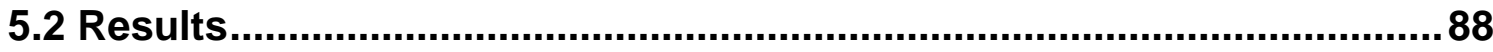




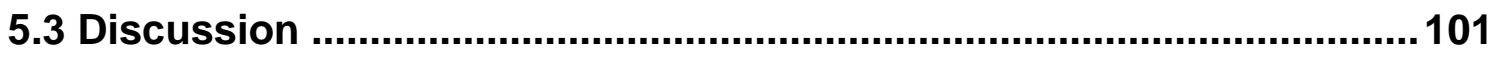

Chapter 6: General discussion............................................... 108

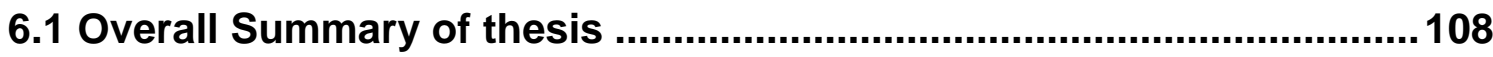

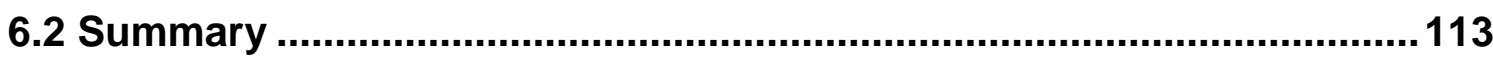

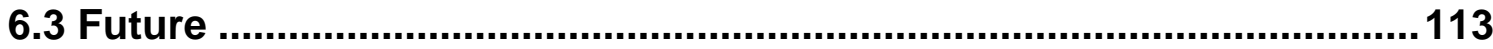

Appendix A-Supplementary Tables and figures......................... 115

Appendix B-Recipes...................................................................... 123

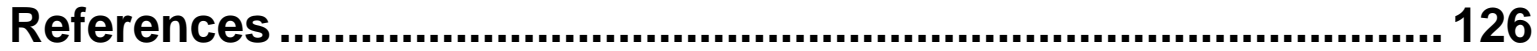

Acknowledgements.................................................................. 145 


\section{Table of Figures:}

Figure 3.1 SRBC:IgG + LPS elicit different cytokine profiles by macrophages than LPS alone.....43

Figure 3.2 Macrophages exposed to SRBC:IgG up-regulate SPHK1 and iNOS expression . .44

Figure 3.3 Type II activation induces the most significant alteration in the IL-10/IL-12 ratio at $8 \mathrm{~h}$ post stimulation

Figure 3.4 Type II activation induces the most significant alterations in surface marker expression at $24 \mathrm{~h}$ post stimulation

Figure 3.5 IL-10 inhibition does not alter the phenotype of type II activated macrophages. .53

Figure 3.6 CD40 stimulation does not alter the phenotype of type II activated macrophages. ........56

Figure 4.1 Type II and classical activation support similar levels of antigen-specific T cell proliferation.

Figure 4.2 Type II and classical activation induce different levels of antigen-specific IL-2 production .72

Figure 4.3 Type II activation reduces Th1 while increasing Th2 responsiveness .75

Figure 4.4 Type II macrophages reduce Th1 development independant of CD40/CD40L interactions

Figure 4.5 CD40/CD40L inhibition reduces Th2 responsiveness by type II macrophages . .79

Figure 4.6 CD40/CD40L inhibition increases IL-10 in response to classical macrophages . .79

Figure 5.1 Schistosome egg and egg complex reduce LPS-induced IL-12 and increase IL-10........90

Figure 5.2 Schistosome egg and egg complex induce no significant alterations in macrophage surface markers

Figure 5.3 Schistosome complex of 5-20 $\mu \mathrm{g} / \mathrm{ml}$ elicits the greatest reduction in LPS-induced IL-12

Figure 5.4 SEA complexes elicit a similar IL-10/IL-12 ratio to type II macrophages but differ significantly in IL-4 induction

Figure 5.5 SEA complexes elicit similar alterations in surface markers to type II macrophages.....96

Figure 5.6 SEA induces SPHK1 expression and iNOS in macrophages ...... .99

Figure 5.7 SEA complexes induce the expression of RELM $\alpha$ in macrophages 100 


\section{List of abbreviations:}

ab -Antibody

Ag- Antigen

ANOVA- Analysis Of Variance

APC- Antigen Presenting cell(s)

BMMØ- Bone marrow derived macrophage

CFSE-5,6-carboxyfluorescein succinimidyl ester

c-Maf- Leucine zipper transcription factor

CNS- Central Nervous System

$\mathrm{CO}_{2}-$ Carbon dioxide

CTCM- Complete T cell medium

CTLA-4- Cytotoxic T Lymphocyte Antigen 4

Cyc- Cychrome

DC - Dendritic Cell(s)

Derp-1- Dermatophagoides Pteronyssinus group1

dPBS- Dulbeccos Phosphate Buffered Saline

EAE- Experimental Autoimmune Encephalomyelits

Egg:Ser- Schistosome egg opsonised with Serum from schistosome infected mice

ELISA- Enzyme-linked immunosorbant assay

eNOS- Endothelial Nitric Oxide Synthase

FACs- Flourescence-activated cell sorting

Fc $\gamma R$ - Immunoglobulin G Receptor (Fc gamma receptor)

FCS- Foetal Calf Serum

Fitc- Fluorescein Isothiocyanate

Foxp3- Forkhead Box P3

GA- Glatiramer Acetate

GATA-3- Trans-acting T cell specific transcription factor

GITR-Glucocorticoid induced tumour necrosis factor receptor family related 
gene

GM-CSF- Granulocyte/Macrophage-Colony Stimulating Factor

IC-Immune complex

IDO- Indoleamine 2,3-dioxygenase

IFN $\gamma$ - Interferon gamma

IgG- Immunoglobulin G

Ig-Immunoglobulin

IL- Interleukin

IL-2R $\alpha$ - Interleukin 2 receptor alpha

IL-4R $\alpha$ - Interleukin 4 Receptor alpha

iNOS- Inducible Nitric Oxide Synthase

iTregs- Inducible Regulatory T cell(s)

LPS- Lipopolysaccharide

MCP-1- Monocyte chemotactic protein-1

MFI-Mean Flourescene Intensity

MHC - Major Histocompatibility Complex

MMP- Matrix metalloprotease

MØ-Macrophage

mRNA- messenger RNA

ms- mouse

MS- Multiple Sclerosis

NFкB- Nuclear Factor-Kappa B

NK cells- Natural Killer

nNOS- Neuronal Nitric Oxide Synthase

NO- Nitric Oxide

nTreg- Natural Regulatory T cell(s)

OVA- Ovalbumin Protein

PBS- Phosphate Buffered Saline

PD-1- Programmed Death-1 
PDL-1- Programmed Death Ligand-1

PDL-2- Programmed Death Ligand-2

PE- Phycoerythrin

PRRs- Pathogen Recognition Receptors

rb- rabbit

RBC- Red blood cell

RELM $\alpha$-Resistin-like molecule-alpha

rIL-10- recombinant interleukin 10

rIL-4- recombinant Interleukin 4

RT- Room temperature

SDS PAGE- Sodium dodecyl sulphate polyacrylamide gel electrophoresis SEA- Schistosome Soluble Egg Antigen

SEA-IgG- Soluble egg antigen opsonised with purified immunoglobulin G SPHK1- Sphingosine Kinase-1

SRBC- Sheep Red Blood Cell

SRBC:IgG- SRBC opsonised with immunoglobulin G

STAT-1- Signal Transducer and Activator of Transcription protein 1

STAT-5-Signal Transducer and Activator of Transcription protein 5

STAT-6- Signal transducer and activator of transcription protein 6

TAM- Tumour Associated Macrophage

T-bet- Member of T-box family of transcription factor expressed in T cells

TCR- T Cell Receptor

TGF $\beta$ - Transforming Growth Factor beta

Th- T helper cell

Th17- T helper 17 cell

Th1-T helper 1 cell

Th2- T helper cell 2

TLR- Toll-like receptor

TNF $\alpha$ - Tumour Necrosis Factor-alpha 
TNFRI- Tumour Necrosis Factor Receptor type 1

TNFRII- Tumour Necrosis Factor Receptor type 2

Tregs- Regulatory T Cell(s)

Ym-1- Chitinase-like secetory lectin

\section{Units and measurements:}

$\mu \mathrm{g} / \mathrm{ml}$ - micrograms per millilitre

h- hour

mM- micro moles

$\mathrm{U} / \mathrm{ml}$ - units per millilitre

$\mathrm{ng} / \mathrm{ml}$ - nonograms per millilitre 


\section{Chapter 1: Introduction}

\subsection{Innate and adaptive immune responses}

Adequate protection against pathogens requires an efficient innate and adaptive immune response (Abbas et al., 1996; Bowdish et al., 2007; Janeway et al., 2002). The innate arm of the immune response is the first to recognise pathogens, create an initial defense, and alert the adaptive arm of the immune response when extra help is required. Cells involved in innate immune responses include macrophages (MØ), dendritic cells (DC), mast cells, basophils, neutrophils and natural killer (NK) cells (Janeway et al., 2002). Innate immune cells express a repertoire of germ-line encoded receptors. Upon recognition of general pathogen associated molecular patterns (PAMPs), innate cells elicit rapid responses such as the phagocytosis of bacteria, uptake of cellular debris and secretion of reactive oxygen species. Innate responses also interact with cells of the adaptive immune system through the presentation of antigen and production of cytokines and mediators which influence the adaptive responses (Vyas et al., 2008). Unlike the innate system, cells of the adaptive immune response elicit more targeted responses against a single antigen to which their assembled receptors are specific (Janeway et al., 2002). Cells of the adaptive immune system include CD4 T cells, NK.T cells, Regulatory T cells, and B cells. Although the responses of the adaptive immune system is slower than that of the innate arm, the cells of the adaptive system can elicit memory responses such that upon re-exposure to the same antigen, a much faster, more effective and stronger response can occur. The innate and adaptive immune systems work together to provide host protection, with each system aiding the other for optimal anti-pathogenic responses (Campbell et al., 1996; Stenger et al., 1996).

\subsection{Macrophages}

\subsubsection{Derivation}

Macrophages are innate immune cells, and are the first line of defense against invading pathogens (Dunn et al., 1985). They are derived from haemopoietic cells that develop into monocytes, which upon entering tissues from the blood, differentiate into macrophages (Brem-Exner et al., 2008; Gordon et al., 2005; Mosser et al., 2008). Macrophages are distributed in essentially every tissue such as the lungs, liver, bone, brain, connective tissue, intestines and skin. Depending on the tissue environment, they are capable of eliciting different physiological functions (Stout et al., 2004). These functions can include host defence, wound repair, or immune regulation (Albina et al., 1990; Anderson et al., 2002b; MacMicking et al., 1997). Macrophages are extremely heterogeneous and exhibit a variety of functions including homeostatic mechanisms such as the recycling of red 
blood cells (RBCs) and removal of debris, as well as functions involving the activation of immune responses against pathogens (Mosser et al., 2008). Much of their immune responses are derived by the recognition of conserved molecular patterns on pathogens, which are detected by pathogen recognition receptors (PRRs) such as scavenger receptors and toll-like receptors (TLR) (Janssens et al., 2003; Pluddemann et al., 2007). There are also receptors such as Fc $\gamma$ Rs and complement receptors which are involved in recognising opsonised particles (Gordon, 2007). Upon recognition of pathogens, macrophages can elicit a variety of functions depending on the receptors involved in the particular response. Receptors such as Fc $\gamma$ Rs, scavenger receptors and complement receptors can elicit phagocytosis (Aderem et al., 1999). In contrast, TLR signalling alters the production of inflammatory mediators involved in immune activation, which in the context of antigen presentation, can initiate appropriate $T$ cell responses. These only represent some functions of macrophages, and each function often crosses over into the other, as seen by the ability of phagocytosis to sometimes induce the production of inflammatory mediators or enhance T cell proliferation (Barker et al., 2002; Janeway et al., 2002).

\subsubsection{Function}

\subsubsection{Phagocytosis}

Phagocytosis is an ancient function of macrophages (Sanjuan et al., 2007), and involves the recognition of pathogen associated molecular patterns (PAMPs) through the expression of PRRs (Akira et al., 2004). These PRRs enable macrophages to recognize and phagocytose particles or pathogens, and include a wide array of different receptors such as scavenger receptors, mannose receptors, Fc $\gamma$ receptors and complement receptors (Aderem et al., 1999; Janeway et al., 2002). In addition to taking up and killing pathogens, macrophages also use phagocytosis for homeostatic mechanisms like the clearance of erythrocytes and apoptotic or necrotic debris (Barker et al., 2002; Mosser et al., 2008). Involved in the degradation of pathogens upon cellular uptake into the phagosome, are processes involved in phagosome maturation, phagosome-lysosome fusion and acidification. Although TLRs are non-phagocytic receptors, they contribute to the phagocytic process through alterations of macrophage responses, and are important in the maturation of phagosomes (Sanjuan et al., 2007). As well as clearing pathogens by uptake and degradation, the phagocytic process can often result in the production of inflammatory mediators, and enhanced expression of co-stimulatory molecules associated with T cell activation (Barker et al., 2002). This is seen by the ligation of mannose receptors and Fcy receptors (Aderem et al., 1999), which either alone or in conjunction 
with TLR signalling can activate the transcription factor NFKB and result in the production of inflammatory mediators (Gordon, 2007).

\subsubsection{Pathogen/danger recognition}

The ability of macrophages to respond to microbial infection lies in the wide array of PRRs capable of recognising different PAMPs, thus enabling responses to numerous pathogens such as bacteria, fungi and viruses (Creagh et al., 2006). PAMPs are conserved molecular motifs that are present on bacteria and helminths, as well as host cells, and enable immune cells to distinguish non-self (pathogen) from self (host cells) (Janssens et al., 2003). These PRRs include TLRs, lectin receptors, scavenger receptors and complement receptors (Pluddemann et al., 2007). Some of these PRRs are involved in the homeostatic mechanisms (e.g scavenger receptors), while others are involved in the activation of macrophages (e.g. TLR) through the production of inflammatory mediators (Janeway et al., 2002; Mukherjee et al., 2009). To date, 13 TLRs have been identified in mice and 11 in humans (Zhang et al., 2008). Different responses can be elicited by the downstream signalling of various TLRs, and some TLRs can co-operate with each other. An example of this is TLR2 co-operating with either TLR1 or 6 to significantly enhance inflammatory cytokine production (Ozinsky et al., 2000). In response to TLR signalling, macrophages produce a vast array of inflammatory mediators (Akira et al., 2004; Janeway et al., 2002).

The most well characterised TLR is TLR4, which is stimulated by bacterial components such as lipopolysaccharide (LPS) from the outer membrane of gram negative bacteria (Akira et al., 2004). TLR4 signalling leads to NFאB activation and results in increased expression of MHC II, the co-stimulatory molecule CD40, as well as the production of proinflammatory cytokines such as IL-6, TNFa and IL-12 (Chow et al., 1999; Gordon, 2007). The production of TNFa in concert with IFN $\gamma$ increases the anti-microbial functions of macrophages by enhancing the production of superoxide anions and nitric oxide (Chow et al., 1999; Hoshino et al., 1999; Modolell et al., 1995). The multitude of cytokines produced can elicit a variety of responses ranging from activation to inhibition of immune responses, depending on the cytokine repertoire induced (Gerber et al., 2001; Janeway et al., 2002; O'Shea et al., 2008; Rahim et al., 2005).

\subsubsection{Other functions}

Macrophages are extremely plastic and multi-functional (Mosser, 2003; Stout et al., 2004). Macrophages are capable of a wide variety of different functions, some of which include tissue remodelling and antigen presentation. In the presence of helminths for example, macrophages alter their functions to produce cytokines and mediators involved in tissue 
remodelling and repair, as opposed to exhibiting anti-microbial functions (Corraliza et al., 1995; Kane et al., 2008; Pesce et al., 2009b). Macrophages involved in wound repair and tissue remodelling express enzymes diametrically opposed to those produced by macrophages involved in responses to danger signals (Modolell et al., 1995). One such enzyme is arginase 1, which converts the substrate for nitric oxide production, into precursors for collagen and polyamines that are involved in the deposition of the extracellular matrix (Corraliza et al., 1995; Modolell et al., 1995).

Another important function of macrophages is the presentation of antigen to $T$ cells in the context of major histocompatability complexes (MHC), to evoke responses of $\mathrm{T}$ cells carrying $T$ cell receptors (TCR) specific for recognising the particular antigen (Russell et al., 2009). Dendritic cells are highly specialised at antigen presentation, however macrophages also contribute to this process. Pathogens are taken up, processed, and presented on the cell surface in the context of MHC I (for intracellular-derived pathogens), or MHC II (for extracellular constituents) (Smith-Garvin et al., 2009). TLR signalling plays an important role in the activation of APCs, resulting in the high levels of expression of MHC molecules, as well as co-stimulatory molecules on APCs, required for the full activation of T cells (Banchereau et al., 1994; Vyas et al., 2008).

Macrophages are highly plastic, and are able to significantly deviate $\mathrm{T}$ cell responses depending on the environmental signals (e.g cytokines and chemokines), which are predominantly produced by macrophages (Attwood et al., 1999; Barker et al., 2002; Curtsinger et al., 1999). Macrophages in an inflammatory state are able to present antigen and induce the development of CD4 T cell subsets of an inflammatory phenotype (Th1,Th17) (Schulz et al., 2009; Seder et al., 1996). Macrophages involved in immune regulation elicit an environment dominated by anti-inflammatory cytokines/mediators which results in more polarized Th2 responses (Anderson et al., 2002a; Gerber et al., 2001). Therefore, macrophages have important roles in directing not only innate responses, but also adaptive immune responses, through the different immune environments elicited by the various macrophage phenotypes.

\subsubsection{Production of immune mediators}

\subsubsection{IL-12}

IL-12 is a pro-inflammatory cytokine most prominently produced by APCs. IL-12 consists of a heterodimer of p40 and p35, which together form IL-12p70 (Oppmann et al., 2000), and is produced by multiple cell types although the major source appears to be from dendritic cells and macrophages (DeKruyff et al., 1997; Kelsall et al., 1996). IL-12 is required for the ability of macrophages to protect the host from intracellular pathogens 
such as Leishmania major, and Mycobacterium avium (Campbell et al., 1996; Florido et al., 2004; Li et al., 1997), with the addition of recombinant murine IL-12 shown to cure such infections (Heinzel et al., 1993). This protection occurs primarily through the induction of Th1 cells producing IFN $\gamma$ (Seder et al., 1993). In concert with CD40 ligation, this induces the activation of macrophages resulting in the production of nitro oxide (NO) and superoxide anions required for parasite killing (Campbell et al., 1996).

\subsubsection{IL-10}

IL-10 is secreted by many different cell types including Th1 cells (Abbas et al., 1996), Th2 cells (Sornasse et al., 1996), Tregs (Groux et al., 1997), and innate cells such as macrophages (Anderson et al., 2002a). Although IL-10 was first thought to suppress immune responses as seen in its suppressive effect on macrophages (Bogdan et al., 1991), it soon became evident that its functions extended beyond simple inhibition. IL-10 has been shown to suppress Th1-associated responses by inhibiting pro-inflammatory cytokines such as IL-6, TNFa, IL-1 $\beta$ and IL-12p40 (Anderson et al., 2004; de Waal Malefyt et al., 1991; Howard et al., 1993). Additionally it is also able to promote the development of regulatory $\mathrm{T}$ cell subsets (Tregs). Furthermore, an essential role for IL-10 in Th2 induction was demonstrated by Groux et al 1997, where IL-10 deficient mice suffered from severe morbidity during helminth infection due to the absence of a protective Th2 response. IL-10 induces the expression of IL-4Ra on macrophages (Lang et al., 2002), which are essential in mediating the protective effects of Th2-derived IL-4 during schistosomiasis. In the context of pro-inflammatory diseases such as endotoxemia and EAE, macrophage-produced IL-10 has been shown to be essential in conferring protection or increasing recovery (Gerber et al., 2001; Howard et al., 1993; Kennedy et al., 1992).

\subsubsection{TNFa}

Tumor necrosis factor alpha (TNFa) is a pro-inflammatory cytokine produced most predominantly by macrophages (Ma, 2001). TNFa production is produced early in response to inflammatory stimuli such as IFN $\gamma$ or LPS (Crume et al., 2007; Vila-del Sol et al., 2007). The autocrine production of TNFa is essential for the activation of macrophages, as seen by the inability of macrophages to elicit anti-microbial functions in the absence of TNFa (Vila-del Sol et al., 2007). This is due, in part, to the activation of transcription factors such as $\mathrm{NF} \kappa \mathrm{B}$, which are required for the production of inflammatory mediators (e.g iNOS), cytokines, and chemokines involved in the recruitment of leukocytes (Ciesielski et al., 2002; Vila-del Sol et al., 2007). Some of the inflammatory consequences of TNFa production include its involvement in septic shock, tumoricidal functions, as well as the pathogenesis of autoimmunities including MS, where high levels 
of TNFa production are seen at the peak of disease (Abu-Amer et al., 1994; Begolka et al., 1998).

In addition to initiating inflammatory responses, TNFa also exhibits regulatory functions. This is seen by the suppressive effects on inflammatory mediators such as IL-12 (Ma, 2001), which are significantly enhanced in the absence of TNF $\alpha$. The functions of TNF $\alpha$ vary, dependant on the environmental setting, the location, as well as the differential signalling pathways elicited through the receptors TNFRI or TNFRII.

\subsubsection{IL-6}

IL-6 is a pleiotropic cytokine that is produced by a variety of cells including T cells, dendritic cells, macrophages, and astrocytes (Akira et al., 1990; Jang et al., 2004; Linkhart et al., 1991; Yasukawa et al., 2003). The functions of IL-6 range from bone resorption to the proliferation of lymphocytes, differentiation of B cells, and the induction of both proinflammatory (Veldhoen et al., 2006) and anti-inflammatory responses (Rincon et al., 1997). IL-6 has been shown to inhibit the production of other pro-inflammatory cytokines such as TNF $\alpha$ and IL-1 $\beta$ by monocytes (Maimone et al., 1997; Tilg et al., 1994). Overall, IL-6 seems to have a role in both the initiation and down-regulation of inflammatory responses. This idea is supported by the induction of IL- 6 by IL-12 early on, but the inhibition of IL-12 and Th1 responses by IL-6 during later stages of schistosomiasis (La Flamme et al., 2000).

\subsubsection{Nitric Oxide}

The production of nitric oxide (NO) occurs through the enzyme nitric oxide sythase (NOS), to which three isoforms exhist; neuronal NOS(nNOS), endothelial NOS (eNOS) and inducible NOS (iNOS) (Alderton et al., 2001). nNOS and eNOS are primarily produced in neuronal or endothelic tissue (Alderton et al., 2001), however most NO occurs primarily through inducible nitric oxide sythase (iNOS). This can be induced in many different cell types although macrophages are believed to be a major producer of NO during immune responses (MacMicking et al., 1997). All three NOS enzymes exhibit different catalytic functions and can lead to various consequences, and this can also be demonstrated in vivo, where iNOS but not eNOS or nNOS is essential for maintaining the production of NO in order to control infection with the intracellular parasite Leishmania spp (Stenger et al., 1996).

\subsubsection{Macrophage activation states}

A recent system of classification was proposed by Mosser et al in 2008 to separate activated macrophages based on their functional phenotypes: host defence, wound 
healing, and immune regulation. Mosser et al (2008) and Stout et al (2004), in a similar review, also emphasize the importance of understanding that macrophages exhibit not only the three currently recognized states of activation (i.e. classical, alternative and type II), but a broad spectrum of activation states. Because macrophages are highly plastic (Stout et al., 2004), and are not simply restricted to a single activation state, there may be distinct macrophage states which exhibit characteristics of two or more activation states, termed 'hybrid' macrophages (Mosser et al., 2008). However, for the purpose of this thesis only the three well-characterized types of macrophage activation states, namely classical, type II (regulatory), and alternative, will be covered.

\subsubsection{Classical}

In response to microbial products, macrophages are activated via TLR signalling pathways, into a state that exhibits anti-microbial actions (Campbell et al., 1996; Janeway et al., 2002). Macrophages activated in this manner are termed classically activated. Classically activated macrophages are efficient at presenting antigen to $\mathrm{T}$ cells, and once activated, can induce both the proliferation of T cells, and Th1 polarisation (Campbell et al., 1996; Mosser, 2003; Seder et al., 1996). In vitro studies inducing classically activated macrophages, often stimulate them into such a state with interferon gamma (IFN $\gamma$ ), followed by LPS (Held et al., 1999; Sutterwala et al., 1997). In vivo, IFN $\gamma$ can be derived from cells of both the innate immune system (e.g. NK T cells), and adaptive immune system (e.g. Th1 cells) (Germann et al., 1993). TNFa is one of the first cytokines produced by classically activated macrophages, with secretion occurring as early as two hours after stimulation (Crume et al., 2007). IFN $\gamma$ can stimulate the production of TNFa by macrophages, which further enhances the anti-microbial functions of classically activated macrophages through increased production of NO (MacMicking et al., 1997; Vila-del Sol et al., 2007). Classical activation requires these two signals, as IFN $\gamma$ primes the cell to enable a full response to LPS (Held et al., 1999). Such macrophages then produce high amounts of pro-inflammatory cytokines (e.g IL-12, TNFa, and IL-6), and chemokines (e.g MCP-9, MCP-1) (Anderson et al., 2002b; Grazia Cappiello et al., 2001; Steube et al., 1999). Classically activated macrophages up-regulate the expression of surface molecules that are involved in antigen presentation, such as MHC II (Edwards et al., 2006), as well as co-stimulatory molecules like CD40 and CD80 which are involved in regulating and maintaining the interaction between macrophages and T cells (Nolan et al., 2008; Qin et al., 2005; Tierney et al., 2009).

During infections with intracellular pathogens, the classical activation of macrophages is essential. For example, infection of macrophages with the intracellular parasite Leishmania major, renders them incapable of producing $\mathrm{NO}$ and killing the parasite. This 
inhibition results in exacerbation of disease (Liew et al., 1990; Stenger et al., 1996). However, although the pro-inflammatory responses induced by classical macrophages are required for protection from invading pathogens, the mediators can be detrimental when the production is uncontrolled. Such an uncontrolled pro-inflammatory response occurs in septic shock, which is associated with the overproduction of TNFa and reactive oxygen intermediates (Victor et al., 1998), or in autoimmunity when the response is inappropriately directed at self antigens. In particular, during the autoimmune disease multiple sclerosis (MS), classically activated macrophages cause damage to the central nervous system (CNS) as a result of IL-23, IL-12p40 and IL-6 production, as well as the production of proteases such as matrix metalloproteases (MMPs) (Becher et al., 2002; Langrish et al., 2005; Tran et al., 1998; Veldhoen et al., 2006).

\subsubsection{Alternative}

In the presence of IL-4, IL-13 or glucocorticoids, macrophages are activated into a state distinct from those which exhibit the classical anti-microbial functions described above (Modolell et al., 1995; Stein et al., 1992). These macrophages are believed to be primarily involved in wound healing rather than microbial killing (Pesce et al., 2009a). Central to this difference in function is the altered metabolism of L-arginine to precursors for collagen synthesis as opposed to NO for microbial killing (Albina et al., 1990; Modolell et al., 1995). Alternatively activated macrophages dominate during infection with Schistosoma spp, and are involved in the generation of granulomas that surround the parasite eggs which become trapped in tissues (Hesse et al., 2001). Alternatively activated macrophages are essential to host survival during schistosomiasis as in their absence, schistosome-infected mice succumb to severe mobidity and death (Herbert et al., 2004).

In addition to their contribution to tissue remodelling, alternatively activated macrophages also differ from classically activated macrophages in several other regards. For instance, these macrophages are not efficient at presenting antigen to $\mathrm{T}$ cells, do not produce significant amounts of NO, and do not produce pro-inflammatory cytokines like IL-12 (Corraliza et al., 1995; Edwards et al., 2006; Schebesch et al., 1997). In contrast, they produce anti-inflammatory cytokines such as IL-10. In vitro and in vivo, alternatively activated macrophages can be identified by their high levels of arginase1 (Hesse et al., 2001), RELMa (FIZZ1) (Raes et al., 2002), and chitinase (YM-1) (Edwards et al., 2006). Furthermore, alternatively activated macrophages do not up-regulate the activation markers CD40, CD80 or MHC II, but can be identified through their high expression of programmed death ligand-2 (PDL-2) (Loke et al., 2003; Tierney et al., 2009). 


\subsubsection{Type II}

A third distinct state of macrophage activation is type II or regulatory activation. In contrast to classical activation where macrophages are stimulated with TLR agonists such as LPS, type II activation occurs when TLR stimulation is combined with ligation of FcyRs by immune complexes (Anderson et al., 2002b; Gerber et al., 2001). These macrophages exhibit anti-inflammatory effects, with increased production of $\mathrm{IL}-10$ and reduced production of IL-12. Unlike alternatively activated macrophages, type II macrophages elicit the activation of $\mathrm{T}$ cells, as demonstrated by increased CD25 expression, and the biasing of $\mathrm{T}$ cell responses towards a Th2 dominated subset (Anderson et al., 2002b; Edwards et al., 2006).

This pathway of activation by Fc $\gamma R$ ligation was the first identified; however, it is important to note that since this first discovery, other means of type II or regulatory macrophage activation have been found. For example, glucocorticoids or IL-10, inhibit the production of pro-inflammatory cytokines by macrophages and enhance anti-inflammatory responses (Elenkov, 2004; Martinez et al., 2008). Also macrophages in the tumor microenvironment termed tumor associated macrophages (TAM) elicit characteristics similar to regulatory macrophages. They produce high levels of IL-10 and low levels of pro-inflammatory cytokines, but do not express iNOS or successfully present antigen to T cells (Dinapoli et al., 1996; Mantovani et al., 2002). Although these conditions can elicit regulatory macrophages, some characteristics differ between regulatory macrophages induced by these different pathways, such as iNOS expression (Martinez et al., 2008). Despite these differences, the main functional characteristic which appears to define regulatory macrophages is the preferential production of IL-10 over IL-12.

The protective, anti-inflammatory effects of type II activated macrophages have been demonstrated in several animal models of pro-inflammatory disease such as septic shock (i.e. lethal endotoxemia) and multiple sclerosis (Gerber et al., 2001; Tierney et al., 2009). In these studies, transfer of type II activated macrophages protected mice from lethal doses of LPS (Gerber et al., 2001; Sutterwala et al., 1998), or CNS inflammation and disease (Tierney et al., 2009).

Although beneficial against pro-inflammatory diseases (Gerber et al., 2001; Tierney et al., 2009), as with classically activated macrophages, excessive regulatory macrophage responses can be detrimental to the host. For example, tumor associated macrophages, through their immunosuppressive effects, promote tumor growth (Lin et al., 2006). Furthermore, during Leishmania infection, high levels of circulating antibodies form 
complexes with Leishmania, therefore eliciting $\mathrm{Fc} \gamma \mathrm{R}$ ligation and inducing type II macrophage activation (Miles et al., 2005). This activation state is not beneficial to the host as it impairs the elimination of the parasite.

\subsection{CD4 T cells}

In the thymus, $T$ cells undergo developmental changes involving the recombination of their T cell receptor (TCR) chains to generate unique TCRs (Swain, 1995). After TCR recombination takes place, $T$ cells undergo processes which result in distinct populations of either CD4 or CD8 T cells ( $\mathrm{Li}$ et al., 2007). Each population is involved in different immunological processes. CD8 T cells (Cytotoxic T cells) are involved in the killing of virally-infected or cancerous cells (Barry et al., 2002), whereas CD4 helper T cells play an important role in providing support to other immune cells such as B cells, macrophages and CD8 T cells, as well as directing and regulating immune responses (Wan et al., 2009).

T cells leave the thymus as naïve cells that have never encountered their TCR-specific antigen. To activate a naive CD4 T cell successfully, at least two signals are required. First, the TCR must recognize a peptide antigen in the context of an MHC II molecule expressed on the APC, and secondly, this interaction must be accompanied by a costimulatory signal (discussed in more detail later) (Lechler et al., 2001). Upon activation, CD4 T cells proliferate, aided by the cytokine IL-2 (Jelley-Gibbs et al., 2000; Swain, 1995). After proliferation, $T$ cells differentiate into effector $T$ cells, capable of producing cytokines and mediators that activate or regulate other immune cells, or become memory $\mathrm{T}$ cells. The effector T cell subset into which naive CD4 T cells differentiate, largely depend on the cytokine environment at the time of activation, as can be determined by the activation state of the antigen presenting cell (Le Gros et al., 1990; Manetti et al., 1993; Mosser et al., 2008; Wan et al., 2009).

CD4 T helper cells can be divided into several subsets based on their different effector functions. Mossman et al (1986) demonstrated the ability of two helper T cell populations (Th1 and Th2) to be identified based on the production of the cytokines IFN $\gamma$ or IL-4, respectively. Furthermore, not only could the $T$ cell subsets be defined by the cytokines they produced, but the cytokines also exhibited counter-regulatory activity on the differentiation of other T cell subsets (Maggi et al., 1992; Parronchi et al., 1992; Seder et al., 1992). Since then, more CD4 T cell subsets have been identified, and the major and most well-defined of which include Th1, Th2, Th17, and regulatory T cells (Mills, 2008; Zhu et al., 2008). As this thesis will focus mainly on T cell differentiation into Th1 and Th2 cells, only these subsets will be discussed in depth. 


\subsubsection{Th1 cells}

Th1 cells function in cell mediated immunity and are essential for protection from intracellular pathogens such as Mycobacterium avium and Leishmania major (Campbell et al., 1996; Florido et al., 2004), as well as eliciting anti-tumour activity (Micallef et al., 1997). However, the same responses which are beneficial during bacterial infections are detrimental when the responses are directed at targeting 'self antigen'. This is seen in the dominant role Th1 cells have in eliciting pro-inflammatory responses during an animal model for multiple sclerosis, experimental autoimmune encephalomyelitis (EAE), and rheumatoid arthritis (Becher et al., 2002; Leung et al., 2000).

IL-12 is an essential cytokine in the development, proliferation and survival of Th1 cells (Manetti et al., 1993; Seder et al., 1993). In the absence of IL-12, such as that seen in mice lacking the IL-12 gene (Magram et al., 1996), or from macrophages defective in IL12 production (Anderson et al., 2002b), there is a severe impairment in type1 responses and the development of Th1 cells. The strength of the ability of IL-12 to induce the development of Th1 cells was demonstrated by Manetti et al (1993), where in the presence of a Th2-promoting antigen-Dermatophagoides pteronyssinus group1 (Derp1)-T cells elicited a Th1 rather than Th2 response after prior exposure to IL-12. At the level of transcription, the commitment to Th1 differentiation occurs via inhibition of GATA-3, a transcription factor associated with Th2 commitment (Nurieva et al., 2003), and the induction of STAT-1/T-bet (Szabo et al., 2000), involved in suppressing Th2 development.

IFN $\gamma$ is the most prominent cytokine derived from Th1 cells and mediates a range of inflammatory responses (Abbas et al., 1996). IFN $\gamma$ activates macrophages into a state which produces higher amounts of toxic free radicals as well as pro-inflammatory cytokines essential to host protection from pathogens (Seder et al., 1993). The interaction of Th1 cells with APCs in the presence of IFN $\gamma$ induces the activation and increased antimicrobial activity of APCs (MacMicking et al., 1997). This interaction further enhances the production of IL-12 and other pro-inflammatory cytokines by the APCs. The production of IFN $\gamma$ not only has important roles in activating macrophages and other APCs, but can also support cytotoxic T cell and B cell responses. Specifically, after exposure to Th1 subsets, the primary antibody isotypes produced by B cells are $\lg 22 a$ and $\lg G 3$, which are isotypes involved in the opsonisation and phagocytosis by activated APCs (Coffman et al., 1993; Manetti et al., 1993).

\subsubsection{Th2 cells}

In an environment dominated by IL-4, CD4 T helper cells develop into the Th2 subset (Le Gros et al., 1990; Seder et al., 1992). There is no single cell type responsible for the 
production of this cytokine, and the main source still remains a mystery. Cells hypothesized to contribute to the production of IL-4 include NKT cells, basophils, eosinophils and Th2 cells (Bjerke et al., 1996; Le Gros et al., 1990; Macaulay et al., 1997). Even in the context of an infection with a Th1 inducing pathogen, L. major, IL-4 administration induces a potent Th2 response (Chatelain et al., 1992). The production of IL-4 by $T$ cells works in an autocrine and paracrine manner, signalling through the IL-4 receptor, a heterodimer of IL-4R $\alpha$ and the common gamma chain (i.e. IL-2R $\gamma$ ). This signal initiates a cascade of signalling events involving STAT-6, which results in the activation of transcription factors driving Th2 commitment (Kaplan et al., 1996). These transcription factors include GATA-3 and c-maf (Kurata et al., 1999), and just as T-bet inhibits IL-4 production, GATA-3 inhibits IFN $\gamma$ production (Ouyang et al., 1998; Szabo et al., 2000).

Other cytokines are also thought to play a role in Th2 differentiation, including IL-2 and IL10. IL-2 was first identified for its effects on promoting $T$ cell proliferation and differentiation (Smith, 1988), and is produced by a variety of cells including Th1 cells. Recently, IL-2 has been identified as a major cytokine determinant of early differentiation into the Th2 subset. Signalling via IL-2Ra induces STAT-5 dependant increased accessibility of the IL-4 locus, required for IL-4 production (Cote-Sierra et al., 2004), and enhances the expression of IL-4R $\alpha$ (Liao et al., 2008), without which, no Th2 development would occur. In addition to the deviation towards a Th2 subset, IL-2 is important in the survival and function of regulatory T cells (Sakaguchi, 2004; Setoguchi et al., 2005). IL-2 was also found important in preventing overzealous inflammatory responses, and was found capable of limiting $T$ cell responses directly through various mechanisms such as the induction of apoptosis, termed activation induced cell death (Jelley-Gibbs et al., 2005). IL-10 is also thought to play a role in Th2 cell biasing, as IL-10 knockout mice are unable to elicit Th2 responses to the Th2-inducing parasitic worm, Schistosoma mansoni (Wynn et al., 1998). Furthermore, macrophages defective in IL-10 production are likewise unable to drive Th2 differentiation in vitro (Anderson et al., 2002b).

The primary cytokines produced by fully differentiated Th2 cells include IL-4, IL- 5 and IL13 (Wan et al., 2009). IL-4 is produced by basophils, NK cells, mast cells, eosinophils, T cells, and macrophages under specific conditions (Mukherjee et al., 2009; Pouliot et al., 2009). Not only does IL-4 induce the development of Th2 cells (Le Gros et al., 1990; Seder et al., 1992), but also activates macrophages into an altered state such that they no longer produce pro-inflammatory cytokines, but instead elicit anti-inflammatory responses involved in wound healing and repair (Albina et al., 1990; Raes et al., 2002). In the setting of pro-inflammatory diseases such as EAE, IL-4 is associated with protection rather than pathogenesis (Begolka et al., 1998; Shaw et al., 1997). 
In contrast to Th1 cells, Th2 cells takes longer to differentiate as demonstrated by the dependency of IL-4 production on cell cycle progression (Bird et al., 1998). Effector Th2 cells have major roles in humoral immunity, elimination of extracellular pathogens (e.g. parasitic helminths) (Sher et al., 1992), and through cytokine production, counter-regulate or "suppress" the pro-inflammatory responses elicited by Th1 or Th17 cells (Zhu et al., 2008). IL-12 and IFN $\gamma$ aid Th1 development and inhibit Th2 responses, whereas IL-10 and IL-4 are potent antagonists to Th1 responses. Furthermore, IL-4 and IL-10 are capable of inhibiting the development and proliferation as well as the effector functions of Th1 cells through the inhibition of a range of pro-inflammatory cytokines involved in driving Th1 responses (D'Andrea et al., 1993; Fiorentino et al., 1991; Murray, 2006). As with Th1 cells, CD4 T cells of the Th2 subset can induce antibody production by B cells, however the predominant antibody isotypes produced are $\lg \mathrm{G} 1$ and $\lg \mathrm{E}$ (Manetti et al., 1993).

Although Th2 responses have been associated with recovery from pro-inflammatory diseases (Gerber et al., 2001; Shaw et al., 1997), as with Th1 responses, the same mechanism which elicits protection can lead to pathology under different environmental settings. For example, excessive Th2 responses induces atopic asthma and dermatitis (Wierenga et al., 1990), and during schistosomiasis, can lead to excessive fibrosis through the production of IL-13 (Herbert et al., 2008; Ramalingam et al., 2009). Thus it is the balance between these diverse immune responses that is critical to maintain.

\subsubsection{Th17 cells}

The discovery that another subset of CD4 T cells was involved in the pathogenesis of the pro-inflammatory disease, EAE, led to the identification of Th17 cells. IL- 6 and TGF $\beta$ enable de novo development of Th17 cells (Veldhoen et al., 2006), and IL-23 plays an important role in the stabilisation of this $T$ cell subset (Langrish et al., 2005). After activation, Th17 cells produce high amounts of IL-6, TNFa, IL-21, IL-22 and IL-17. Unlike Th1 cells, they produce very little IFN $\gamma$ (Jager et al., 2009; Langrish et al., 2005; Stockinger et al., 2007). Effector Th17 cells elicit anti-pathogenic roles, eliminating various infections with which Th1 cells require help, such as Leishmania, Salmonella and Mycobacterium tuberculosis (Wan et al., 2009) . Th17 cells also promote pro-inflammatory autoimmune responses and have been implicated in the pathogenesis of EAE, psoriasis and T cell mediated Colitis (Langrish et al., 2005; Yen et al., 2006).

\subsubsection{Regulatory $\mathrm{T}$ cells}

Another distinct subset of CD4 T cells are regulatory T cells (Tregs). Naturally occurring regulatory T cells (nTregs) develop in the thymus (Sakaguchi, 2004; Wan et al., 2009). However naive CD4 T cells can also be induced to develop into a regulatory state in the periphery, termed inducible Tregs (iTreg) (Zhu et al., 2008). Naturally occurring Tregs 
express high levels of CD25 (IL-2Ra), glucocorticoid induced tumour necrosis factor receptor family related gene (GITR), and the transcription factor FOXp3 (Bettelli et al., 2006; Nakamura et al., 2001), with FOXp3 being a widely used marker to identify nTregs (Fontenot et al., 2005). FOXp3 was found to not only act as a marker, but also play a role in the development of nTregs in the thymus (Fontenot et al., 2003). Although the discovery of FOXp3 in nTregs has been extremely beneficial in their identification, it has also become clear than FOXp3 can also be induced in non-nTreg effector T cells, through STAT-5 signalling (Roncarolo et al., 2008). Inducible Tregs can be induced by IL-10 and TGF $\beta$ (Zhang et al., 2010). Unlike nTregs, iTregs do not constitutively express FOXp3, but can do so after induction, and only express it transiently.

Upon activation, Tregs produce cytokines such as IL-10 and TGF $\beta$, and suppress T cell responses (Sakaguchi, 2004). The suppression of T cell responses is believed to occur through a range of mechanisms. For example, high levels of CD25 (IL-2R $\alpha$ ) on Tregs can induce $\mathrm{T}$ cell death, due to deprivation of IL-2 in the environment which is required for CD4 T cell survival (Pandiyan et al., 2007). Also, apoptosis can be induced by the production of granzymes (Vignali et al., 2008). Immune responses can also be suppressed by the inhibitory cytokines IL-10 and TGF $\beta$, and through the ability of Tregs to alter the function of APCs to produce immunosuppressive molecules such as indoleamine 2,3-dioxygenase (IDO), which directly inhibits T cell proliferation (Munn et al., 1999). Overall, Tregs function primarily to promote self tolerance and prevent exacerbated inflammatory responses (Vignali et al., 2008). The role of Tregs in limiting self reactive immune responses has been seen in mice and humans, where a lack of Tregs results in severe autoimmune lymphoproliferative diseases. Patients with mutations with FOXp3, suffer from x-linked diabetes melitis, and enteropathy (Wildin et al., 2001).

\subsection{Macrophage-T cell Interactions}

Macrophages are one of the main professional APCs in addition to DCs and B cells. This process results in the direct interaction between the macrophage and $\mathrm{T}$ cell in an antigenspecific manner as described in Section 1.2.2.3. Although the recognition of peptide in the context of a self-MHC molecule by the T cell's TCR forms the foundation of this interaction, other molecules are important in determining the outcome of this exchange in terms of the level of $\mathrm{T}$ cell activation, the type of subset differentiation, or type of macrophage effector functions. These molecular interactions include but are not limited to CD28:CD80/86, CD40L:CD40, and PD-1:PDL-1/PDL-2.

\subsubsection{CD28/CD80 co-stimulation}

Naïve T cells cannot be activated by TCR recognition of peptide bound to MHC alone, but instead require a second signal - co-stimulation (Lamb et al., 1983; Lechler et al., 2001). 
The first co-stimulatory molecules to be identified were CD80 and CD86, which are expressed on APCs, and their T cell-expressed ligands, CD28 and CTLA-4 (Linsley et al., 1991). The interaction between CD28 and CD80 or CD86 sends a positive signal and leads to T cell activation (Alegre et al., 2001), while CTLA-4 binding to CD80 or CD86 sends an inhibitory signal and limits T cell activation (Waterhouse et al., 1995). CD28 is constitutively expressed on T cells, however co-signalling molecules alter their levels of expression depending on the environment. Therefore, APCs presenting self antigen to self-reactive $T$ cells in the absence of activation, should not be expressing high levels costimulatory molecules. In this circumstance, $\mathrm{T}$ cells are not receiving the required activation signals and instead become un-responsive (Lechler et al., 2001). This is beneficial in the prevention of autoimmunities. Conversely, TLR (or another PRR) stimulation, which occurs during an infection, results in the up-regulation of CD80 or CD86 on APCs and thus promotes the activation of antigen-specific naïve $T$ cells to fight the invading organism (Janeway et al., 2002).

\subsubsection{The dual role of CD40/CD40L co-stimulation}

Another co-signalling molecule is CD40 present on B cells, macrophages, monocytes, platelets, astrocytes and endothelial cells. This molecule was identified in 1986 as a costimulatory molecule involved in the induction of B cell growth and isotype switching (Armitage et al., 1993; Banchereau et al., 1994; Clarke, 2000). Its' ligand, CD40L (CD154), is present on activated T cells, and was identified in 1992 by Armitage et al. The interaction between CD40L on T cells and CD40 on B cells is essential for isotype switching by $B$ cells. This was demonstrated in patients with a genetic mutation of CD4OL termed X-linked hyper IgM syndrome, whereby B cells were unable to elicit isotype switching. They only produced IgM antibodies, which resulted in recurrent infections (Hill et al., 1993). However the role of the CD40/CD40L interaction goes beyond B cells, and has also shown to have important roles in the activation of many cell types including $T$ cells, monocytes/macrophages and epithelial cells (Grewal et al., 1998; Grewal et al., 1996).

CD40/CD40L interaction is important in both T cell and APC activation (Stout et al., 1996b). In the absence of CD40L, antigen-specific $T$ cells show reduced capacity to produce cytokines in response to antigen in vitro (Grewal et al., 1998), implicating this interaction as playing a role in the induction of $\mathrm{T}$ cell unresponsiveness in the periphery. Ligation of CD40 on macrophages and monocytes via CD40L, induces the up-regulation of cytokines/chemokines and surface markers associated with the induction of $\mathrm{T}$ cell responses, and cell-mediated immunity (Stout et al., 1996a; Toes et al., 1998). For example, CD40 ligation by CD40L up-regulates CD80, MHC II and cytokines such as TNFa, IL-6, and IL-12 (Alderson et al., 1993; Campbell et al., 1996; Caux et al., 1994), 
involved in promoting inflammatory Th1 responses. The CD40/CD40L interaction not only enhances the ability of APCs to activate T cells through the production of IL-12 and expression of MHC II, but is also essential for the induction of anti-microbial activity and activation of the APC itself (Campbell et al., 1996; Stout et al., 1996b). Th1-derived IFN $\gamma$ along with CD40/CD40L signalling increases the production of nitric oxide (NO) (Tian et al., 1995), essential for the killing of intracellular pathogens. CD40/CD40L interactions can also induce the up-regulation of other co-stimulatory molecules such as CD80/86 on B cells, making them more efficient at presenting antigen and stimulating $\mathrm{T}$ cells ( $\mathrm{Wu}$ et al., 1995).

The role of CD40/CD40L in directing $T$ cell subset differentiation has been under intense investigation, as some studies have demonstrated the role is not strictly limited to the induction of Th1 responses. The production of prototypic Th1 and Th2 cytokines (IFN $\gamma$ and IL-4 respectively) are significantly abrogated in the absence of CD40L in mice; implying CD40 plays a role in both Th1 and Th2 immune responses (Poudrier et al., 1998). This has been further supported by MacDonald et al (2002), who demonstrated in vitro and in vivo that the CD40/CD40L interaction between the $T$ cell and DC was essential to induce an immune response by Th2- but not Th1-inducing stimuli (MacDonald et al., 2002). In addition, Hancock et al (1996) found that administration of anti-CD40L antibody resulted in a reduction in IFN $\gamma$ and IL-12, and an increase in Th2 associated cytokines IL-4 and IL-10, suggesting that CD40/CD40L alterations can promote Th2 induction. Therefore, while it seems CD40 is involved in both Th1 and Th2 responses, many studies also support a preferential role for CD40 in Th2 biasing.

This reciprocal role of CD40 in regulating both Th1 and Th2 responses may be down to the level of signalling received. Indeed, corroborating with this is in vitro data by Mathur et al (2004), performed on primary microglia and macrophage cell lines. Mathur et al demonstrate high levels of CD40 signalling induces the production of the pro-inflammatory cytokine IL-12, while low level signalling elicits a reduction in IL-12 and increase in the anti-inflammatory cytokine IL-10. Thus CD40/CD40L signalling represents a single interaction which elicits dual counter-regulatory roles. The exact mechanisms involved in inducing these reciprocal responses are unknown, and studies are currently underway. In this thesis, the mechanisms involved in altering the CD40/CD40L interaction and the effects of this altered interaction on macrophage as well as CD4 T cell phenotypes are investigated.

\subsubsection{PD-1/PDL-1 co-signalling}

Co-inhibitors as well as co-stimulators exist to limit the extent of immune activation. Like CTLA-4, Programmed Death 1 (PD-1) is an inhibitor of T cell responses. PD-1 was first 
identified in 1992 by Ishida et al, in studies on the programmed death of cells. PD-1 is not constitutively expressed, but as with CTLA-4, is induced by T cell activation. Its' role in peripheral tolerance and prevention of autoimmunites has become apparent, with PD-1 deficient mice suffering from spontaneous autoimmunities such as lupus-like arthritis (Nishimura et al., 1999), and autoimmune mediated cardiomyopathy (Nishimura et al., 2001b).

There are two ligands for PD-1; Programmed Death ligand 1 and 2 (PDL-1 and PDL-2) (Freeman et al., 2000; Latchman et al., 2001), and the functions of these ligands remain controversial. Some studies indicate the PD-1 ligands inhibit $\mathrm{T}$ cell activation and proliferation (Freeman et al., 2000), however there have also been conflicting evidence that they are involved in T cell stimulation (Tseng et al., 2001). Other studies have shown that the PD-1 ligands may inhibit the response of APCs through reverse signalling as seen in DCs (Kuipers et al., 2006). PDL-1 is constitutively expressed on a range of cells including $T$ cells, DCs, B cells and macrophages as well as non-haemopoietic tissue, with its up-regulation occurring by exposure to inflammatory stimuli such as LPS (Yamazaki et al., 2002). PDL-2, a second ligand for PD-1 (Latchman et al., 2001) is less widely expressed, and its expression on DCs and macrophages only occurs after stimulation with cytokines such as IFN $\gamma$, GMCSF, or IL-4 (Loke et al., 2003; Yamazaki et al., 2002). PDL-1 and PDL-2 exhibit some overlapping functions, where both induce inhibition of $T$ cell responses as exhibited by reduced IFN $\gamma$ and IL-2 by T cells (Keir et al., 2006). The ligands for PD-1 seem to exhibit functions both similar and distinct from each other, having effects not only on the inhibition/stimulation of $\mathrm{T}$ cell responses, but also having diametrically opposed roles in Th1/Th2 responses (Loke et al., 2003), implying they may elicit differential roles in deviating immune responses.

\subsection{Schistosomiasis}

\subsubsection{Lifecycle}

More than 250 million people are affected by schistosomiasis world wide (Chitsulo et al., 2000). Schistosomiasis is infection with the trematode worm of the schistosoma genera (Butterworth et al., 1994). The species which cause the majority of infection in humans are S.mansoni, S.japonicum, and S. Haematobium. All are endemic in tropic and subtropical equatorial areas (Kurtzke, 2000), and although the risk of mortality is less than that of other infectious diseases such as M.tuberculosis, schistosomiasis as with other helminth diseases, results in severe long lasting morbidity (Ouma et al., 2001).

The infective cercariae derived from fresh water snails residing in contaminated water, penetrate the skin of humans while gathering water, catching fish, or washing. After 
travelling through the blood stream, matured worms reside in the hepatic portal vasculature where they mate and produce their eggs about 5-6 weeks post infection (Dunne et al., 2005). Eggs must then traverse the intestine to exit out through the feces (S.mansoni, S.Japonicum) or pass through the urine in the case of urinary schistosomiasis caused by S. Haematobium (Hu et al., 2004). After deposition of the eggcontaining feces or urine into the waterways, miracidia hatch from the eggs and infect the snail host continuing the infectious cycle.

\subsubsection{Schistosomiasis immunology}

Although the schistosome worm itself can live for up to 40 years undetected by the immune system (Chabasse et al 1985 as cited in Dunn et al., 2005 and Hu et al., 2004), the eggs they produce induce a strong inflammatory response which is required for the eggs to exit out through the intestine/bladder to continue their life cycle. However, many eggs get lodged in organs such as the liver (S.mansoni) where they reach a dead end in their life cycle progression. The inflammatory response which ensues is largely driven by polarised Th2 responses (Grzych et al., 1991; Pearce et al., 2004), which orchestrates a granulomatous/fibrotic response at these foci of eggs (Pearce et al., 2004). This Th2 driven response is both essential for host protection, and at the same time is the cause of host pathology. In S.mansoni infection, IL-4 prevents excessive damage to the liver induced by reactive oxygen radicals produced in response to bacterial components which seep out of the intestine, as shown in IL-4 deficient mice which suffer severe liver damage associated with significant increases in NO (La Flamme et al., 2001), as well as in mice deficient in the IL-4 receptor (Herbert et al., 2004). However, in excess, the same response can be detrimental to the host. At the site of granuloma formation, mediators and cytokines in the local environment, derived predominantly by macrophages, induce Th2 cells to secrete IL-4 and IL-13, which are pro-fibrotic cytokines (Wynn et al., 2004). As well as having important roles in altering macrophage and $T$ cell responses into an antiinflammatory state and perpetuating responses involved in tissue remodelling, IL-4 and IL13 also directly induce collagen synthesis from fibroblasts (Jakubzick et al., 2003; Sempowski et al., 1994) which when in excess, results in severe pathologies as seen during the chronic stages of schistosomiasis. Such pathologies include liver fibrosis, hepatosplenomegaly, the development of portal ascites, as well as portal hypertension, all of which may lead to organ failure (Ouma et al., 2001; Pearce et al., 2004). However, the immune responses to eggs aid in the movement though the gut/bladder wall for those destined to those sites. It also prevents systemic deposition of trapped eggs within the liver/intestine and through its counter regulation of Th1 responses, prevents exacerbated Th1-driven inflammation (Herbert et al., 2008; Pearce et al., 1991). Therefore, Th2 
dominant immune responses, although pathology-inducing, are essential for host protection from severe schistosomiasis.

\subsubsection{Macrophage activation during Schistosomiasis}

Macrophages and DCs are altered in the presence of schistosome egg or soluble egg antigen (SEA). Both DCs and macrophages show a significant reduction in the ability to promote Th1 responses and produce pro-inflammatory cytokines such as IL-12 (La Flamme et al., 2004; Zaccone et al., 2003). This effect of reducing the activation in response to pro-inflammatory conditions occurs independently of MyD88 in DCs, a characteristic signalling molecule involved in the production of pro-inflammatory cytokines (Kane et al., 2008). In the presence of LPS in vitro, which is a likely contaminant during infection with schistosomiasis, macrophages exposed to SEA produce lower levels of the pro-inflammatory cytokine IL-12 and increased production of IL-10 (La Flamme et al., 2004). Therefore, SEA can induce cytokine deviation at the level of the macrophage, such that Th1 inducing cytokines are reduced while Th2 or Treg-inducing cytokines are increased. This ability to counter-regulate the response to LPS is likely a mechanism which occurs in vivo, in the setting of contaminating bacterial components which seep out of the intestine from pores created by the traversing of eggs through the gut wall (Herbert et al., 2008).

Alternatively activated macrophages are essential for host survival during schistosomiasis (Herbert et al., 2004), and IL-4 in the absence of alternatively activated macrophages does not elicit protection, indicating macrophages as the major elicitors of IL-4 induced protection. Further studies identified that in the absence of IL-4Ra on bone marrow derived cells such as macrophages, severe mortality ensues (Herbert et al., 2008). This was associated with severe inflammation in the liver, which may have been caused by the lack of anti-inflammatory mediators and cytokines, and the removal of the suppressive effect of alternatively activated macrophages on T cell responses (Herbert et al., 2008). As well as alternatively activated macrophages inducing Th2 responses, they are also capable of suppressing these same Th2 responses, and this dual effect is essential in maintaining a balance of Th2 induced protection and Th2 induced pathology (Nair et al., 2009; Pesce et al., 2009a).

The effect of schistosome infection on type II activation is currently unknown. As with type II macrophage activation (Tierney et al., 2009), schistosome infection either with worm, egg, or soluble egg antigen (SEA), protects mice against EAE (La Flamme et al., 2003; Sewell et al., 2003; Zheng et al., 2008). Furthermore, some of the responses associated with protection are similar. As with type II activation, protection by schistosome infection is dependent on immune deviation resulting in polarised Th2 responses, and reductions in 
Th1 responses (La Flamme et al., 2006; La Flamme et al., 2003; Sewell et al., 2003; Tierney et al., 2009; Zheng et al., 2008). Schistosome egg antigen complexes elicit greater anti-inflammatory responses than schistosome egg antigen alone, with high levels of IL-10 and reduced production of IL-12 (La Flamme et al., 2004), which is a characteristic shared by type II macrophage activation (Anderson et al., 2002b). During infection, schistosome eggs are potent inducers of inflammation, aiding in the exit of eggs into the intestine across the gut wall (Pearce et al., 2004). This results in increased intestinal permeability, and as the intestine is a reservoir of bacteria, leakage of bacterial components such as LPS occurs. Furthermore, this is accompanied by high levels of inflammatory mediators such as IFN $\gamma$ and IL-12 (Herbert et al., 2008). In addition to the presence of IFN $\gamma$ and LPS, schistosomiasis is also accompanied by circulating immune complexes (de Jesus et al., 2002; Santoro et al., 1979). Type II activation is induced by exposure to immune complexes in the presence of inflammatory mediators such as LPS and IFN $\gamma$ (Gerber et al., 2001). Therefore schistosome infection elicits an environment in support of type II macrophage activation, and it is therefore possible such an activation state occurs in vivo. 


\subsection{Objectives of this investigation}

It is essential to identify different macrophage phenotypes as particular phenotypes are associated with disease pathogenesis, or more importantly, with disease prevention/recovery. To date, the number of distinct markers for differentiating type II activation from other regulatory states is quite limited. Furthermore, very little is understood in terms of the mechanisms involved during type II activation, and whether this activation state contributes to the protection schistosome infection provides against EAE.

This thesis investigates the effects of schistosome immune complexes on macrophage activation with the hypothesis that schistosome immune complexes are able to induce type II macrophage activation, similarly to opsonised SRBCs. Understanding the effects of schistosome products on macrophages, and whether the induction of type II activation is involved, will provide further insight into the possible mechanisms involved in protection from inflammatory diseases.

This thesis also investigates mechanisms involved in promoting the type II macrophage phenotype. In particular, the role CD40 alterations have in the effector functions elicited by type II activation is investigated. The hypothesis is that low levels of CD40 present on type II macrophages promote the anti-inflammatory profile elicited by this activation state and are involved in T cell biasing in favour of the Th2 subset.

The goal of this investigation is to provide a greater understanding of mechanisms involved in anti-inflammatory responses elicited by macrophage activation, with the notion that a greater understanding of mechanisms involved in immune deviation will aid the development of more effective treatments for autoimmunities. 


\section{Chapter 2: General Methods}

\subsection{Animals}

All experiments involving animals were approved by the Victoria University Animal Ethics Comittee (AEC). C57BL/6 mice were obtained from and bred at eitherthe Biomedical Research Unit of the Malaghan Institute of Medical Research (MIMR), or the Otago School of Medicine Biomedical Research Unit, Wellington, New Zealand.

Transgenic OTII mice with a transgenic TCR for OVA ${ }_{323-339}$ on a C57BL/6 background (Robertson et al., 2000) were generously donated by lan Hermans and Franca Ronchese, and were bred at the Biomedical Research Unit of the Malaghan Institute of Medicine, Wellington, New Zealand.

Following guidelines specified by the ministry of agriculture and forestry (MAF), mice were housed at Victoria University of Wellington in the animal facility. Mice aged 6-12 weeks were used for experiments following AEC guidelines.

\subsection{Primary Cell culture}

\subsubsection{General Macrophage culture}

Bone marrow derived macrophages (BMM $\varnothing$ ) were cultured from C57BL/6 mice. After euthanasia, mice were soaked with $70 \%$ ethanol as were the instruments used for dissection. With the use of a $23 \mathrm{~g}$ needle and syringe, bone marrow was flushed from the femurs and tibias with divalent cation free Dulbecco's PBS (dPBS-Invitrogen, Auckland, $\mathrm{NZ}$ ) with collections made into a $50 \mathrm{ml}$ falcon tube (BD Bioscience, Franklin Lakes, NJ, USA). Cells were broken apart by homogenisation, washed by centrifugation at $760 \mathrm{xg}$ in wash buffer (Appendix B), followed by lysis of red blood cells with $2 \mathrm{ml}$ of RBC lysing solution (Sigma, St Louis, MO, USA) for 2 minutes. After further washing cells in wash buffer, cells were then seeded at $10^{6} \mathrm{cells} / \mathrm{ml}$ in T75 tissue culture flasks (BD Bioscience, Franklin Lakes, NJ, USA) in complete T cell media (CTCM-In appendix B). After overnight culture at $37^{\circ} \mathrm{C}$ in $5 \% \mathrm{CO}_{2}$, adherent cells were removed and the remaining progenitor cells were cultured on $90 \mathrm{~mm}$ non-tissue culture-treated, gamma-sterilized petri dishes ( Raylab, NZ ) in CTCM with $5 \mathrm{ng} / \mathrm{ml}$ rGMCSF and rlL-3 (Peprotech, N.J ) at $37^{\circ} \mathrm{C}$ in $5 \%$ $\mathrm{CO}_{2}$ for 8-10 days until fully differentiated into macrophages. Cells were then removed by carrying out several rounds of incubation in ice-cold dPBS at $4^{\circ} \mathrm{C}$ followed by vigorous pipetting.

For stimulation assays, macrophages were cultured in 96 well plates (BD Bioscience) at $10^{5}$ cells/well in CTCM, and were primed overnight in $20 \mathrm{U} / \mathrm{ml} \mathrm{IFN} \gamma$ (BD Biosciences) 
followed by exposure to the various culture conditions (as specified in figures). Macrophages were stimulated with $200 \mathrm{ng} / \mathrm{ml}$ lipopolysaccharide (LPS- Sigma) in the presence or absence of opsonised sheep red blood cells (SRBC-lgG), un-opsonised SRBC (Taylor Preston Limited), opsonised schistosome whole egg, un-opsonised schistosome egg (Dalton et al 1997), opsonised soluble egg antigen (SEA), or unopsonised SEA (Boros et al 1970). Cells were incubated at $37^{\circ} \mathrm{C}$ in $5 \% \mathrm{CO}_{2}$.

\subsubsection{Generation of immune complexes}

\subsubsection{SRBC immune complexes}

For each batch of new sheep red blood cells (SRBC), an agglutination assay was carried out in order to determine the optimal concentration of rabbit anti-SRBC IgG (Sigma, St Louis, MO, USA) for opsonisation. Two fold serial dilutions of rabbit anti-SRBC antibody were made in round bottom 96 well plates (BD Bioscience) starting with a 1:50 dilution in 1xPBS (Appendix B). Fresh SRBCs were added to all wells at a final concentration of $10^{8}$ cells $/ \mathrm{ml}$, and incubated at room temperature for 1-2 hours. The highest non-agglutinating concentration was determined by visualising the plate under a light source. Optimal concentrations ranged between 1:200 to $1: 400$ of rabbit anti-SRBC IgG.

Opsonised SRBCs were generated by incubating SRBCs $\left(10^{8}\right.$ cells $\left./ \mathrm{ml}\right)$ with rabbit antiSRBC antibody at predetermined non-agglutinating titres. Incubation of the SRBCs and antibody was carried out at room temperature with rotation of the mixture for $30-40$ minutes. Cells were then washed and the remaining SRBC-IgG complex was added to the macrophage culture at a ratio of 10 SRBC: 1 macrophage.

\subsubsection{Schistosome whole egg immune complexes}

Schistosome whole egg complex was generated by incubation of 1000 Schistosoma mansoni eggs with serum from schistosome infected C57BL/6 mice at a 1:100 dilution of serum. The solution was incubated at room temperature while rotating, for 30 minutes. Following incubation, non-bound serum was removed by washing the solution in PBS, and resuspending the remaining schistosome egg-complex at the desired concentration such that there were 1000 eggs per well.

\subsubsection{Schistosome soluble egg antigen (SEA) immune complexes}

Antibody ( $\lg G$ ) from schistosome infected mice was purified on a protein $G$ sepharose column (Sigma), by Dr J. Tierney. To obtain the SEA-immune complex, SEA (either 5 or $10 \mu \mathrm{g} / \mathrm{ml}$ ) was incubated with anti-SEA lgG (either 10 or $20 \mu \mathrm{g} / \mathrm{ml}$ ) in $1 \times \mathrm{xdPBS}$. The solution was incubated for 1 hour while rotating at room temperature. 


\subsubsection{Obtaining single cell splenocyte suspensions}

After removal from OTII C57BL/6 mice, spleens were mechanically mashed using the back of a $1 \mathrm{ml}$ plunger belonging to a $1 \mathrm{ml}$ syringe (BD Bioscience), through $70 \mu \mathrm{m}$ cell strainers (BD Bioscience) into $50 \mathrm{ml}$ falcon tubes to create single cell suspensions. After washing in wash buffer followed by CTCM (Appendix B), splenocytes were incubated for 2 minutes in red blood cell lysing solution. Cells were washed at $760 x g$ centrifugation and resuspended in CTCM at a desired concentration for CD4 T cell isolation.

\subsubsection{Isolation of CD4 T cells from OTII positive mice using Dynabeads and DETACHaBEAD (Brief outline of manufacturer's instructions)}

Splenocytes from OTII positive mice were isolated (described above) and resuspended at $1 \times 10^{7}$ cells/ml in isolation buffer (Appendix B). Mouse CD4 (L3T4) Dynabeads (Invitrogen Dynal, Norway) were washed and added to the resuspended cells at $25 \mu \mathrm{l}$ per $1 \times 10^{7}$ splenocytes, and incubated for 20 minutes while rotating at $4^{\circ} \mathrm{C}$. CD4 negative $\mathrm{T}$ cells were then removed by carrying out several rounds of placing the tube on a DynaMag magnet (Invitrogen) for 2 minutes before discarding the supernatant and resuspending the cells in $100 \mu \mathrm{l}$ CTCM per $1 \times 10^{7}$ cells. The remaining bead-bound CD4 T cells were isolated by adding $10 \mu$ of mouse CD4 DETACHaBEAD (Invitrogen) per $1 \times 10^{7}$ cells, and incubated while rotating at room temperature for 45 minutes. The CD4 T cells were then isolated by placing the tube on the magnet and collecting the supernatant. This was carried out for several rounds to obtain a maximum amount of CD4 T cells. These were then resuspended at the desired concentration for later use in functional assays or flow cytometry (specified in figures). For stimulation assays with macrophages, the CD4 T cells were plated at approximately $1 \times 10^{6}$ cells $/ \mathrm{ml}\left(2.5 \times 10^{5} \mathrm{cells} /\right.$ well $)$ in 96 well plates containing the macrophage cultures, and incubated at $37^{\circ} \mathrm{C}$ in $5 \% \mathrm{CO}_{2}$ for 72 hours.

\subsection{CFSE staining protocol of CD4 T cells.}

CD4 T cells, purified as described above (2.2.4), were resuspended in sterile 1xdPBS at a concentration of $2 \times 10^{7}$ cells $/ \mathrm{ml}$ before incubating with Carboxyfluorescein succinimidyl ester (CFSE, Invitrogen) used at $625 \mathrm{nM}$. After incubation for 8 minutes at room temperature while wrapped in foil, an equal volume of $100 \%$ foetal calf serum (FCS- ICP biologicals, Auckland) was added to quench the CFSE and terminate the reaction. Cells were thoroughly washed to remove extracellular CFSE by several rounds of centrifugation in CTCM, after which cells were resuspended at the desired concentration of $2.5 \times 10^{6}$ cells/ml prior to culture in 96 well plates containing macrophage cultures. 


\subsection{Cytokine detection}

\subsubsection{Enzyme linked immunosorbant assay (ELISA)}

To determine levels of cytokines in the supernatants collected from cell cultures, a sandwich enzyme-linked immunosorbent assay was carried out following manufacturer's recommendations (BD Pharmingen, Franklin Lakes, NJ). All ELISA antibodies were purchased from BD Bioscience unless otherwise stated. In brief:

The cell culture supernatant was removed and stored at $-20^{\circ} \mathrm{C}$ until used. 96 well ELISA plates were coated with $50 \mu \mathrm{L}$ of capture antibody specific to the cytokine of interest (specified in figures). Dilutions of capture antibody were made in capture buffer before transfer to the ELISA plate according to the manufacturer's guidelines for that antibody (See Appendix A table1). ELISA plates were incubated at $4{ }^{\circ} \mathrm{C}$ overnight.

Plates were then flicked to remove any unbound antibody in solution, and incubated in blocking solution ( $1 \mathrm{x}$ dPBS containing $5 \%$ FCS) at room temperature for approximately 2 hours to block non-specific binding. Plates were washed 2-3 times in a washing solution of 1xPBS (Appendix B) containing $0.05 \%$ Tween-20 (Sigma) (TPBS), after which samples were added to the wells either as neat or diluted in blocking solution. Cytokine standards were added to the top two rows in 2 fold serial dilutions with the concentration of the first well specified in appendix A. Plates were incubated for either 2 hours at room temperature, or overnight at $4^{\circ} \mathrm{C}$.

After washing plates in TPBS approximately 4 times, biotinylated detection antibody (BD Bioscience) was diluted in blocking solution at the recommended concentration, and $50 \mu \mathrm{l}$ was added to the wells. Plates were incubated for 1 hour at room temperature. Plates were then washed in TPBS, and incubated in Streptavidin-HRP (SA-HRP-BD Bioscience) for $30 \mathrm{~min}-1$ hour depending on the cytokine of interest.

Directly after washing plates in TPBS, equal volumes of substrate reagent $A$ and $B$ (BD Bioscience) were added to each well and allowed to develop at room temperature under visual supervision. The substrate induced a colour change which allowed one to be able to decide the appropriate time to stop the reaction, at which time $0.18 \mathrm{M}$ sulphuric acid (STOP solution) was added to each well.

Absorbance of each well was then read at $450 \mathrm{~nm}$ using a VersaMax plate reader.

\subsubsection{Cytometric Bead array (CBA)}

For the detection of MCP-1, IL-6, and TNFa in addition to IL-10 and IL-12, a CBA mouse inflammation kit (BD Bioscience) was performed as per manufacturers instructions. 
In brief:

BD Standards were prepared in BD assay diluent, with a range of $0-1667 \mathrm{pg} / \mathrm{ml}$. The 'mixed capture beads' were prepared by mixing together $2.5 \mu \mathrm{l}$ aliquots of each of the 6 capture beads per well to be analysed, in a 1:1 dilution with assay buffer. BD PE detection was diluted in assay buffer in a 1:1 dilution. Following this, $25 \mu$ of the 'Capture bead mix' was added to all wells in a 96-well round bottom plate. This was followed by the addition of $25 \mu \mathrm{l}$ of samples and $25 \mu \mathrm{l}$ of standards to the appropriate wells. Additionally, $25 \mu \mathrm{L}$ of the diluted PE detection was added to all wells, followed by a 2 hour incubation at room temperature in the dark. The plate was then washed in BD wash buffer (BD Bioscience) two times, prior to resuspending the bead-sample pellet in $300 \mu \mathrm{l}$ of wash buffer for analysis on the flow cytometer as reported in 2.5 .

\subsection{Flow Cytometry}

Cells were harvested from 96 well plates at the end of the culture period. For macrophage cultures, cells from duplicate cells were combined in order to acquire enough cells for flow cytometry. Prior to staining, cells were washed to remove excess CTCM. The Fc receptors of cells were blocked by incubation with rat anti-CD16/32 Fc block $(1 \mu \mathrm{g} / \mathrm{ml})$ for 10 minutes. Cells were stained with fluorescently labelled antibodies (BD, Serotec, eBioscience-Appendix B) at dilutions determined by either optimisation within the lab, or recommended by the manufacturers guidelines. Cells were stained and incubated with the fluorescently labelled antibodies for the markers of interest as well isotype controls following the manufacturers recommendations (BD Pharmingen). After staining for markers of interest, cells were analysed using the FACScan flow cytometer (BD Bioscience). For analysis of primary macrophages, 900-1500 live events were collected per assay. For examination of splenocytes or CD4 T cells, 5000-10,000 live events were collected.

The live cell population was determined using the forward scatter (FSC) and side scatter (SSC) data of the non-stained population, which was gated around. Isotype controls and single stains were used to determine background fluorescence and compensation respectively.

Analysis of results was carried out using the CELLQuest pro software (BD, Franklin Lakes, NJ, USA). 


\subsection{Western Blot}

\subsubsection{Obtaining lysate}

Macrophages were removed 9 hours following primary stimulation under the different culture conditions (in figures). Supernatant was removed and macrophages were incubated for 5 minutes in trypsin (TrypLE express, Invitrogen) at $37^{\circ} \mathrm{C}$ in $5 \% \mathrm{CO}_{2}$. Fetal calf serum (FCS-ICP biologicals, Auckland) was added to quench the reaction. Cells were washed two times to remove excess trypsin, with the final wash in 1xdPBS. Cells were then incubated with $100 \mu \mathrm{l}$ of lysing buffer (10 $\mu$ l of protease inhibitor cocktail (Sigma) per $1 \mathrm{ml}$ of RIPA buffer (Appendix B)) for 1 hour at $4^{\circ} \mathrm{C}$ while shaking. The solution was then centrifuged at $2000 x$ for 25 minutes to remove debris. Protein concentrations were assessed using a BCA kit (Pierce). The supernatant containing proteins of interest was removed and stored at -20 or $-80^{\circ} \mathrm{C}$ for future assessment.

\subsubsection{Running western blot}

Preparation of lysates for running in gels was carried out as follows; $80 \mu \mathrm{g}$ of each lysate (or $22 \mu \mathrm{l}$ max) from 2.6.1 were transferred into fresh tubes, and incubated with $8 \mu \mathrm{l}$ of the reducing buffer mix (1:10 dilution of 2-mercaptoethanol (Sigma) in $5 \times$ reducing buffercontaining bromophenol blue in running buffer), at $90^{\circ} \mathrm{C}$ for 10 minutes.

SDS PAGE (Sodium dodecyl sulfacte polyacrylamide gel electrophoresis ) was performed with $30 \mu \mathrm{l}$ of lysates containing reducing buffer along with $3 \mu \mathrm{l}$ of dual colour ladder (Biorad). This was run at $200 \mathrm{~V}$ for 45 minutes (Appendix $B$ for gel recipe). Proteins were transferred onto a PVDF membrane (GE Healthcare, Amersham) by running the transfer for 4 hours at $50 \mathrm{~V}$ in a $4^{\circ} \mathrm{C}$ cold room. Immunoblots were removed and blocked using $5 \%$ non-fat skim milk in TTBS (Appendix B) for 1 hour at room temperature. Membranes were then probed with the primary antibody of interest (Dilutions in Appendix A table2). Membranes were probed with the primary antibody for 2 hours at room temperature, or overnight at $4^{\circ} \mathrm{C}$. This was followed by $3 \times 5$ minute washes in TTBS. Membranes were probed with a secondary fluorescently labelled antibody against the species for which the primary antibody was derived from, for 2 hours at room temperature. Depending on the primary antibody, this was anti-rabbit cy3, or anti-mouse cy5 (GE Healthcare). Immunoblots were washed with $3 \times 5$ minute washes in TTBS. Images of the probed membranes were then obtained on a Fuji Film FLA-5100 scanner. The same membranes were then washed with $3 \times 5$ minute washes in TTBS and the same process was repeated for each primary antibody used. Dependant on the experiment, the primary antibodies included rabbit anti-SPHK1 (Abcam), rabbit anti-RELMa (Abcam) or mouse anti-iNOS 
(Transduction laboratories). Scanned images were analysed using Image Quant. Western blots were normalised to $\alpha$ tubulin.

\subsection{Graphing and statistical analysis}

Results were analysed and graphed in GraphPad, Prism v. 4.0 for Windows (GraphPad Software, San Diego California USA, www.graphpad.com). Statistical analysis was carried out using one way ANOVA or two-way ANOVA with Bonferroni correction. 


\section{Chapter 3: Defining key mechanisms of type II macrophage activation}

\subsection{Introduction}

Macrophages are highly plastic innate cells capable of altering their physiology and therefore their functions depending on changes in the micro-environment (Stout et al 2004). As such, they are able to elicit a broad spectrum of activation states (Mosser et al., 2008) of which the major states include classical, alternative, and type II. Macrophages activated in response to pro-inflammatory stimuli are termed classically activated macrophages. These macrophages elicit cell mediated, Th1-promoting pro-inflammatory responses (Liew et al., 1990; Stenger et al., 1996), and can be characterised by high levels of NO, essential in the killing of intracellular pathogens, as well as the production of inflammatory cytokines and chemokines such as IL-12, TNFa, IL-6 and MCP-1 (Steube et al., 1999). Also associated with the activation of classically activated macrophages is the expression of co-signalling molecules like CD80 and CD40, which are up-regulated during inflammation (Alderson et al., 1993; Caux et al., 1994b; Tierney et al., 2009). In the absence of co-stimulatory markers, cell-mediated responses are significantly impaired (Campbell et al., 1996; Florido et al., 2004). This phenotype has been shown to be detrimental in autoimmunities such as MS, and reductions in the level of macrophages exhibiting classical activation, or inhibition of the inflammatory mediators produced by these macrophages, are associated with protection against CNS inflammation (Cua et al., 2003; Huang et al., 2001b; Sewell et al., 2003).

In 1992 Stein et al identified a novel macrophage state induced by IL-4, which elicited distinct functions from that of classically activated macrophages (Stein et al., 1992). Unlike classically activated macrophages, alternatively activated macrophages convert L-arginine into precursors for collagen synthesis and are involved in tissue remodelling and wound repair as opposed to pathogen killing (Raes et al., 2002). This pathway is in contrast to the conversion of L-arginine to NO by iNOS in classically activation macrophages (Modolell et al., 1995). Also expressed in alternatively activated macrophage is RELMa, which along with arginase 1, exhibits roles in wound repair as well as the suppression of overzealous inflammatory responses (Nair et al., 2009; Pesce et al., 2009a; Pesce et al., 2009b). Alternatively activated macrophages are poor at presenting antigen to T cells, unlike classically activated macrophages (Edwards et al., 2006), and are more inhibitory than stimulatory of T cell responses (Schebesch et al., 1997). 
Adding to the repertoire of macrophage subsets was the discovery of type II activated macrophages (Anderson et al., 2002; Gerber et al., 2001; Sutterwala et al., 1997). These macrophages were classed under the same category as alternatively activated macrophages, due to their differences from classically activated macrophages; however the pathway to activation is completely different. Type II activation occurs via the ligation of Fc $\gamma$ receptors in the presence of LPS and significantly alters the responses to inflammatory stimuli by reducing the expression of co-stimulatory markers (Tierney et al., 2009) and deviating the cytokine profile to promote anti-inflammatory cytokines such as IL-10, over pro-inflammatory cytokines such as IL-12 (Gerber et al., 2001). Unlike alternatively activated macrophages, type II macrophages express iNOS and can be distinguished from classically and alternatively activated macrophages by high expression of the enzyme sphingosine kinase 1 (SPHK1) (Edwards et al., 2006). Furthermore, type II macrophages have the ability to present antigen to $T$ cells and induce Th2 cell biasing (Anderson et al., 2002; Lucas et al., 2005). It is this ability to elicit a Th2 polarized immune response that is believed to counter-regulate Th1-mediated inflammatory responses and provide protection against inflammatory diseases such as endotoxemia and EAE, an animal model of MS (Gerber et al., 2001; Tierney et al., 2009). However, the exact mechanisms involved in type II activation and how these effects provide protection against EAE are currently unknown. Because type II activation is a strong polarizer of antiinflammatory Th2 responses, understanding the mechanisms involved in the ability of type II activation to deviate immune responses would provide greater understanding of how to increase or prevent beneficial or pathological processes respectively.

Aims:

To better understand the mechanisms involved in type II activation, we examined several aspects of type II macrophage activation. These aspects included assessing changes in cytokines and surface markers over a period of time following stimulation, and the involvement of IL-10 and CD40 in regulating the phenotypic alterations in type II macrophages. Overall, there were three specific aims to be addressed:

1. To assess the kinetic changes in the expression of co-signalling molecules and cytokine profiles, in order gain an insight into the kinetics involved in type II activation.

2. Investigate the contribution of IL-10 in the ability of type II macrophages to reduce inflammatory cytokines and surface markers, to determine whether the increase in IL-10 is essential for this macrophage phenotype. 
3. Study the role of CD40 signalling, with regards to the phenotypic changes elicited by type II activation, and investigate whether increased signalling impairs the antiinflammatory phenotype.

\subsection{Results}

\subsubsection{Ability of opsonised SRBCs to induce type II macrophage activation}

In order to investigate the mechanisms involved in type II macrophage activation, we first characterized the phenotype of the type II activated macrophages in our experimental system. Additionally, we wished to identify broader changes occurring in response to type II activation to aid in a greater understanding of how type II activation protects against EAE as well as other inflammatory disorders. For all experiments, we used opsonised sheep red blood cells (SRBCs) in the presence of LPS to induce type II macrophage activation, while LPS alone or in the presence of un-opsonised SRBC was used to induce classical activation. The production of various cytokines was assessed $8 \mathrm{~h}$ following stimulation.

SRBC or SRBC:IgG (opsonised SRBC) in the absence of LPS elicited similar effects to medium alone with respect to cytokine production (IL-10, IL-12, MCP-1, IL-6, TNFa; Figure 3.1). Moreover, stimulation with SRBC in the presence of LPS elicited a similar level of cytokine production to that seen by stimulation with LPS alone, indicating SRBC alone has no effect on macrophage activation (Figure 3.1). In contrast to the effect of unopsonised SRBC, the effects seen by opsonised SRBC in the presence of LPS differed significantly from LPS stimulation alone. For example, IL-10 production in response to SRBC:IgG + LPS is significantly higher than that induced by LPS or SRBC + LPS (unopsonised). Furthermore, macrophages stimulated with LPS produced high levels of IL$12 \mathrm{p} 40$ while the addition of SRBC:IgG to cultures with LPS resulted in a significant reduction in IL-12. These results confirm that the addition of SRBC:IgG has induced type II as opposed to classical macrophage activation in our system.

Exploring the effect of type II activation on the production of other cytokines, we assessed MCP-1, IL-6, IL-4, and TNFa. MCP-1, also known as CCL2, was up-regulated by macrophages stimulated with LPS, and this level of production remains largely unaffected by the addition of SRBC, with only a slight reduction observed. However, MCP-1 levels are reduced by the addition of SRBC:IgG to LPS-stimulated cultures. Although statistically non-significant, this is similar to the effect seen on IL-12 production. LPS stimulated macrophages produce very high levels of IL-6, and in contrast to the effects seen on IL10, MCP-1 and IL-12, SRBC in the presence of LPS appear to reduce the production of IL-6, to a similar degree to that observed by SRBC:IgG + LPS (Figure 3.1). This finding 
indicates the reduction in LPS-induced IL-6 is not due specifically to the ligation of Fcy receptors, but rather due to an unknown mechanism involving the presence of SRBC. Similarly, high levels of TNFa are also produced by LPS stimulated macrophages, but appear to be reduced by the presence of un-opsonised SRBC in LPS cultures. Interestingly, no reduction in TNFa is observed by stimulation of macrophages with LPS in the presence of SRBC:IgG. However as the data are from only 1 experiment, further investigation is required to confirm these results since this effect has not been reported previously. In response to LPS, macrophages produced nearly undetectable levels of IL-4 similar to non-activated macrophages (Figure 3.1). Macrophages stimulated with LPS in the presence of SRBC:IgG showed a higher production compared to non-activated and LPS-activated macrophages, however the difference was not statistically significant. These cytokine profiles indicate stimulation with LPS in the presence of SRBC:IgG increases the production of IL-10, while reducing pro-inflammatory cytokines IL-12 and MCP-1, as well as having effects on IL-6 and possibly TNFa. MCP-1 reductions by type II activation have not been previously reported, nor have reductions in any other chemokines. As chemokines such as MCP-1 contribute to the pathogenesis of inflammatory illnesses, further investigation into the effects of type II activation on chemokines is warranted.

In order to further validate the activation state induced upon stimulation with LPS in the presence of SRBC:IgG, the enzymatic profile of macrophages activated either with LPS or LPS + SRBC:IgG was assessed via western blotting. The levels of iNOS and sphingosine kinase 1 (SPHK1) enzymes were normalised to the expression of $\alpha$ tubulin, one of an array of house-keeping proteins. Figure 3.2 shows the normalised \% of control for the expression of iNOS and SPHK1 in comparison to the levels seen in macrophages stimulated with LPS alone. Macrophages stimulated with LPS have high levels of iNOS indicating successful induction of classically activated macrophages (Figure 3.2), and as expected, macrophages stimulated with LPS + SRBC:IgG express similar iNOS levels as LPS alone (figure 3.2). Sphingosine kinase 1 (SPHK1), a marker for type II macrophage activation, is significantly up-regulated in macrophages stimulated by LPS in the presence of SRBC:IgG compared to stimulation with LPS alone, indicating the induction of type II macrophage activation. As with the cytokine profile, results from the western blot further support the notion that our system of activating macrophages with opsonised SRBC plus LPS induces type II macrophage activation. The ability of these type II activated macrophages to enhance anti-inflammatory cytokines (e.g. IL-10) while reducing its inflammatory counterparts (e.g. IL-12p40 and MCP-1), along with the enhanced expression of SPHK1 generates a viable means to assess type II macrophage activation for further studies within this project. Finally, this work is the first report of type II 
macrophages reducing the expression of $\mathrm{MCP}-1$, or any chemokine, therefore illustrating type II macrophages reduce a wider but selective range of inflammatory mediators than IL-12. 


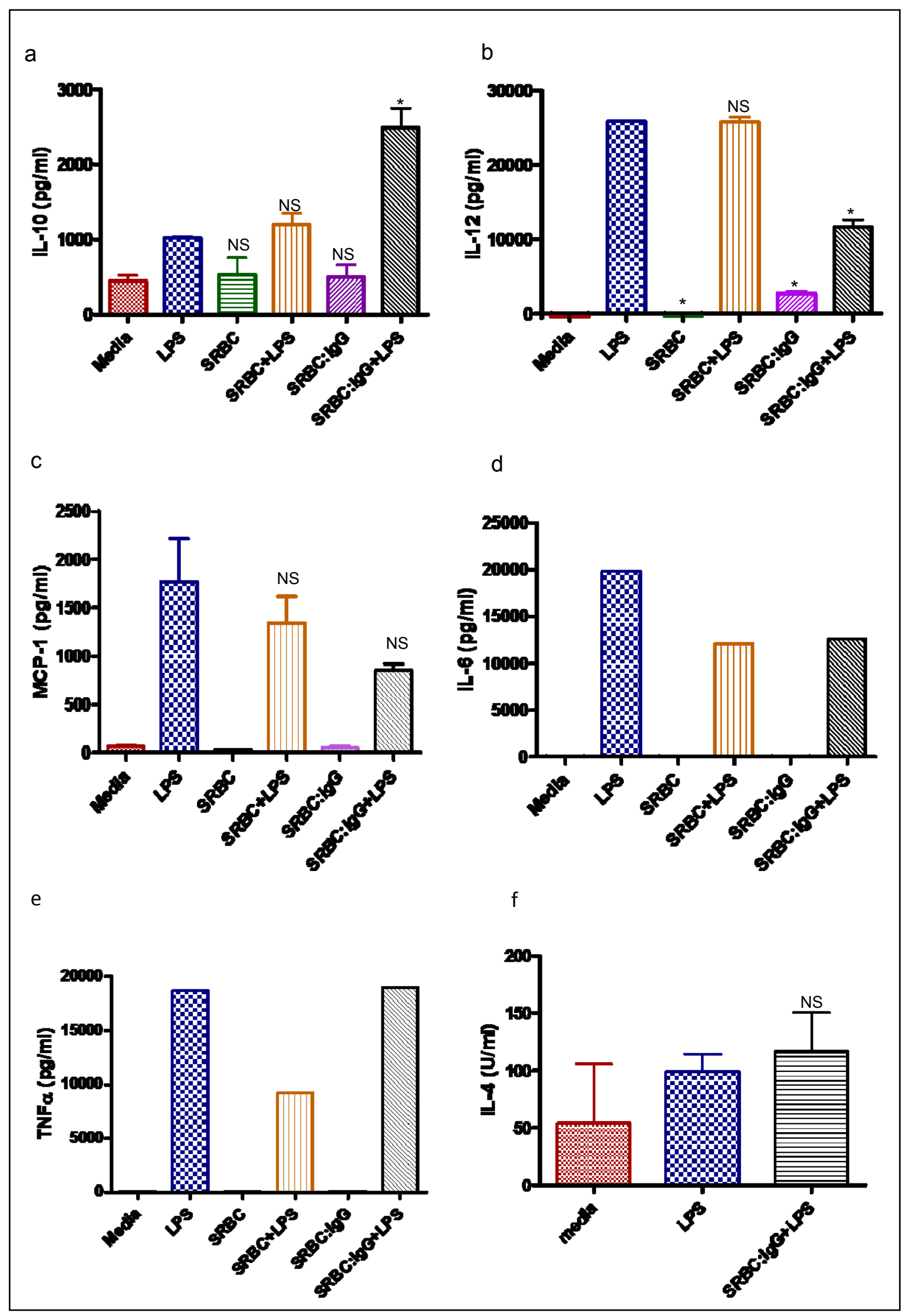

Figure 3.1 SRBC:IgG + LPS elicit different cytokine profiles by macrophages than LPS alone.

Primary BMMØ $\left(10^{6} \mathrm{cells} / \mathrm{ml}\right)$ derived from C57BL/6 mice were primed with $20 \mathrm{U} / \mathrm{ml} \mathrm{IFN} \gamma$ overnight followed by stimulation with un-opsonised SRBC (ratio of 10 SRBC: $1 \mathrm{M \varnothing}$ ), opsonised SRBC 
(SRBC:lgG), $200 \mathrm{ng} / \mathrm{ml}$ LPS alone, or LPS plus un-opsonised or opsonised SRBC. After $8 \mathrm{~h}$ culture, IL-10 (a), IL-12 (b), MCP-1 (c), IL-6 (d), TNFa (e) and IL-4 (f) production were measured via ELISA or a CBA assay (sec 2.4). ${ }^{*} \mathrm{P}<0.05$ : One way ANOVA followed with Bonferroni post test; Conditions versus LPS. Data points (a-c) represent mean +SEM of combined duplicate wells of two experiments. (D-F) represent data points of single wells, resulting in no error bars. (F) represents a single ELISA containing samples for the three conditions of Medium, LPS and SRBC:IgG+LPS.

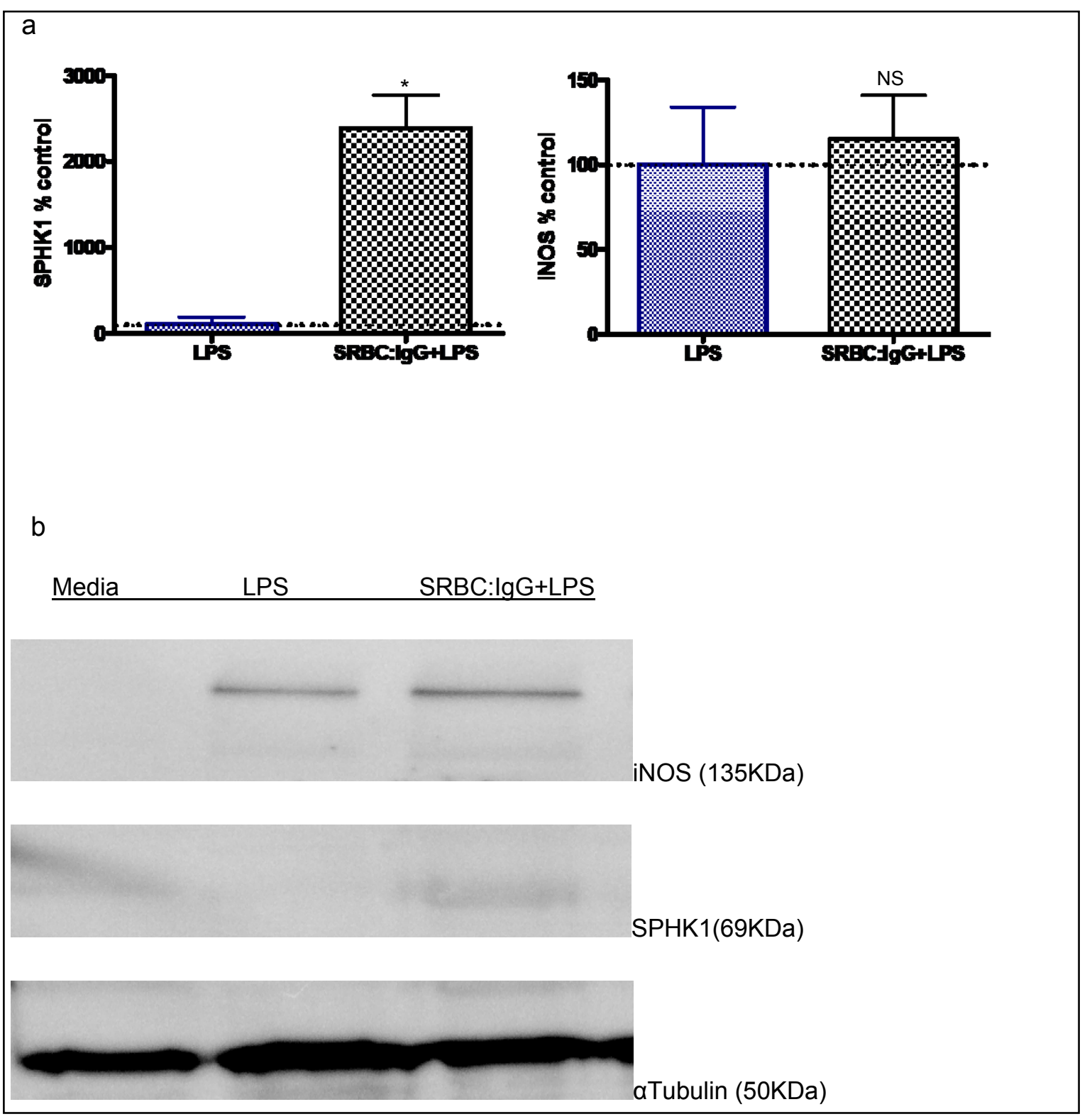

Figure 3.2 Macrophages exposed to SRBC:IgG up-regulate SPHK1 and iNOS expression. Primary BMM $\varnothing\left(10^{6}\right.$ cells $\left./ \mathrm{ml}\right)$ derived from C57BL/6 mice were primed with $20 \mathrm{U} / \mathrm{ml}$ IFN $\gamma$ overnight followed by stimulation with $200 \mathrm{ng} / \mathrm{ml}$ LPS (classical-inducing) or opsonised SRBC plus LPS (10 per MØ) (type II-inducing). After $9 \mathrm{~h}$ culture, expression levels of SPHK1, iNOS and aTubulin were assessed via western blotting (sec 2.6) with anti-SPHK1 pAb (1:700), anti- $\alpha$ Tubulin pAb (1:1000), and antiiNOS pAb (1:700). Band densities were normalised to the expression of $\alpha$ tubulin, and presented as a $\%$ control compared to LPS. ${ }^{*} \mathrm{P}<0.05$ : One way ANOVA followed with Bonferroni post test; 
SRBC:IgG+LPS versus LPS. Data represents the mean+SEM of 2-3 experiments. Diagram (b) is a single membrane representative of the membranes assessed to derive graph (a). 


\subsubsection{Kinetics of cytokines and co-stimulatory markers altered by type II macrophage activation}

Previous studies identified that type II activated macrophages not only alter their cytokine profile, but also significantly change the expression of surface markers in response to inflammatory stimuli (Tierney et al., 2009). Other than the study by Tierney et al, there has been only one other report of alterations in surface markers (Edwards et al., 2006). In order to gain an understanding of possible mechanisms involved during type II macrophage activation, this thesis investigated the kinetics of cytokine and surface marker alterations by assessing changes over a period of time after classical of type II macrophage stimulation. By understanding which alterations occur at which time, the aim was to identify mechanisms involved in determining the type II activated macrophage phenotype.

Represented in this section (Figure 3.3-3.4) are the effects seen by the addition of various stimulants in the presence of LPS. Unless otherwise stated, the effects of SRBC or SRBC:IgG in the absence of LPS mimic that seen by the media controls. Figure $3.3 \mathrm{a}-\mathrm{b}$ show the changes in IL-10 and IL-12 concentration over time, while Figure $3.3 \mathrm{c}-\mathrm{d}$ show the same conditions represented as a \% of control (LPS alone). Unless otherwise stated, there was very little difference in the response of macrophages to LPS stimulation in the presence of SRBC and stimulation with LPS alone. From 2-8 $\mathrm{h}$, the production of IL-12 by classically activated macrophages steadily increased with high levels maintained $24 \mathrm{~h}$ post-stimulation. Production of this pro-inflammatory cytokine was significantly reduced by type II activation (SRBC:IgG+LPS) at $24 \mathrm{~h}$ post stimulation. Additionally, at $8-24 \mathrm{~h}$ post stimulation, type II activated macrophages produced higher levels of IL-10 than LPS stimulation alone, and these levels peaked at $8 \mathrm{~h}$ post stimulation. Together these results indicate that the changes in cytokine production are apparent at all time points post stimulation and are not a result of an accelerated time course in the type II activated cultures.

In addition to cytokine assessments, the expression of CD40, CD80, MHC II, and PDL-1 was assessed at the different time points via flow cytometry (Figure 3.4). Data is presented as a \% control (a-d) in comparison to classical activation (LPS), with the raw data in a2-c2 representing changes over time for all conditions. Exposure of macrophages to SRBC in the presence of LPS induced the same levels of expression of surface markers to that of LPS stimulation alone. Classically activated macrophages (LPS alone) had high levels of CD40 present on their surface, and as with IL-12, levels of CD40 increased over time with the highest levels measured $30 \mathrm{~h}$ post-stimulation (Figure 3.4). By $8 \mathrm{~h}$ post stimulation, differences in CD40 expression were observed. Type II 
macrophages (SRBC:IgG+LPS) had higher levels of CD40 than non-activated macrophages, which barely expressed CD40, but had significantly lower expression of CD40 compared to LPS stimulation. Moreover, the levels of CD40 on type II activated macrophages remained constant 8-24 $\mathrm{h}$ post stimulation, whereas by $30 \mathrm{~h}$ post stimulation, the levels of CD40 on type II macrophages began to increase towards the levels expressed on classical macrophages. In terms of CD80 expression, macrophages exposed to LPS or medium alone exhibited the same levels of expression. Type II macrophages (SRBC:IgG+LPS) had levels of CD80 significantly lower than levels seen on classically activated macrophages, and the levels on these macrophages remained nearly constant over time from 8-30 $\mathrm{h}$ with the greatest \% reduction in comparison to LPSstimulation occurring at $24 \mathrm{~h}$ post-stimulation. PDL-1 expression occurred much later than the expression of CD40 and CD80 on activated macrophages. By $24 \mathrm{~h}$ of culture, significant changes are observed with LPS-treated macrophages exhibiting increased expression of this surface marker. Type II activated macrophages showed reduced expression of PDL-1 at 24 and $30 \mathrm{~h}$ post stimulation with the most notable reduction in PDL-1 compared to LPS-stimulation occurring $24 \mathrm{~h}$ after stimulation.

By $8 \mathrm{~h}$ after stimulation, up-regulation of $\mathrm{MHC} \|\left(\mathrm{I}^{\mathrm{b}}\right)$ expression is observed on all macrophages treated with LPS. No real difference in the levels of MHC II on macrophages from the three different treatment groups were seen until 24 and $30 \mathrm{~h}$ post stimulation. Exposure to SRBC:IgG significantly reduced the expression of MHC II in response to LPS, most significantly at 24-30 h of culture. This data is representative of only one experiment for the $8-30 \mathrm{~h}$ post stimulation time points. Therefore, caution must be used in drawing conclusions.

Observations from these kinetic analyses indicate alterations in cytokine production occur at an earlier time point than the alterations in expression of co-signalling markers. This finding may suggest a causal relationship whereby the increased IL-10 or reduced IL-12 plays an important role in the alterations of CD80, CD40, and PDL-1 induced by type II macrophage activation. Understanding mechanisms involved in perpetuating the phenotype elicited by type II macrophage activation will expand our knowledge on mechanisms involved in the induction of anti-inflammatory responses. 


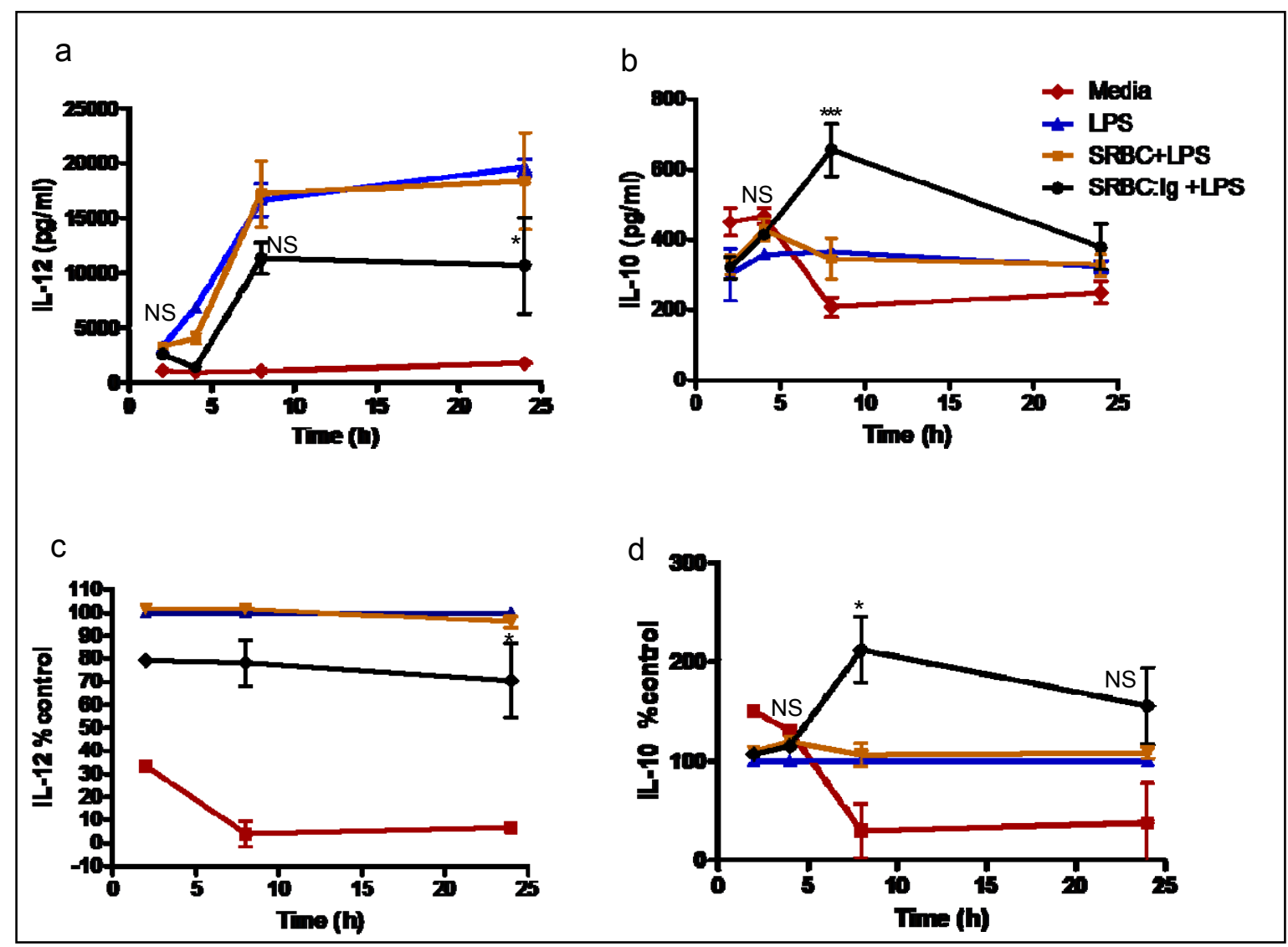

Figure 3.3 Type II activation induces the most significant alteration in the IL-10/IL-12 ratio at $8 \mathrm{~h}$ post stimulation. Macrophages derived from C57BL/6 mice were primed overnight with $20 \mathrm{U} / \mathrm{ml}$ IFN $\gamma$ and stimulated with $200 \mathrm{ng} / \mathrm{ml}$ LPS alone (classical-inducing stimuli) or LPS in the presence of SRBC or opsonised SRBC (type II-inducing stimuli). Cells were also left non-stimulated in media. After 2, 4, 8, and $24 \mathrm{~h}$ culture, IL-12 $(\mathrm{a}, \mathrm{c})$ and IL-10 (b,d) were assessed via ELISA. (c-d) represent the compilation of data, presented as \% compared to LPS (classical activation). ${ }^{*} P<0.05{ }^{* *} P<0.01$ ${ }^{* *} P<0.001$ : Two way ANOVA with Bonferoni post test; SRBC:IgG+LPS versus LPS. Data points represent the mean+SEM of two experiments (c-d), or duplicate wells from one experiment (a-b). 


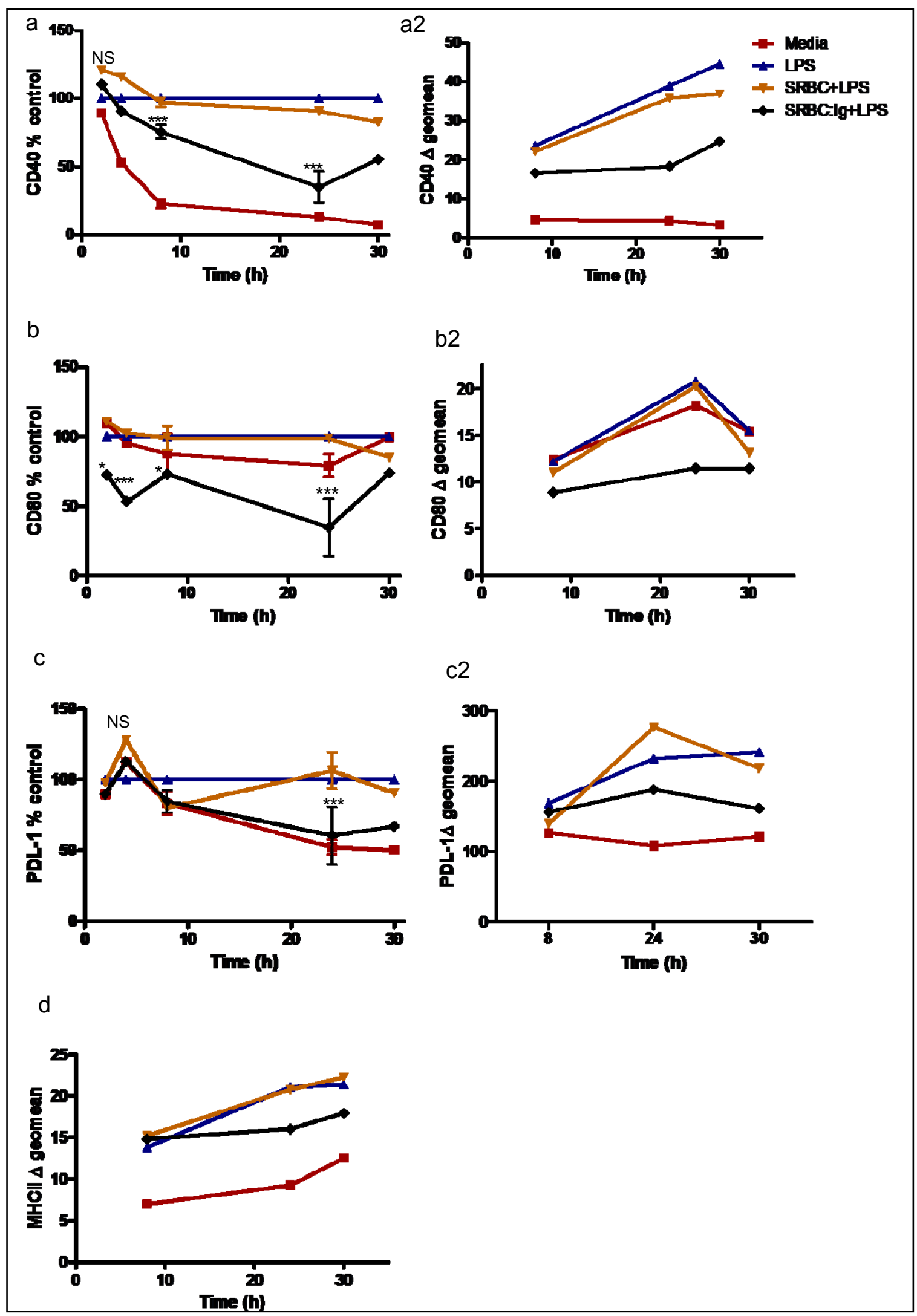

Figure 3.4 Type II activation induces the most significant alterations in surface marker expression at $24 \mathrm{~h}$ post stimulation. Macrophages derived from C57BL/6 mice were treated as described in 
Figure 3.3. After 2, 4, 8, 24, and $30 \mathrm{~h}$ culture, CD40 (a), CD80 (b), PDL-1 (c) and MHC II (d) were assessed via flow cytometry. ${ }^{*} \mathrm{P}<0.05,{ }^{* *} \mathrm{P}<0.01,{ }^{* * *} \mathrm{P}<0.001$ : Two way ANOVA with Bonferroni post test; SRBC:IgG+LPS versus LPS. Data points represent the mean +SEM of two experiments, but in some instances (d) data points represent a single experiment. 


\subsubsection{Involvement of IL-10 in the type II macrophage profile}

The kinetic analysis (sec 3.2.2) revealed that type II activation results in significant increases in IL-10 and reductions in IL-12 prior to reductions seen in the expression of cosignalling molecules. Previous literature emphasizes the suppressive effects of IL-10 on inflammatory cytokines/chemokines such as TNFa, IL-12 and MCP-1 (de Waal Malefyt et al., 1991), with reports of IL-10 also reducing the expression of CD40 in response to LPS (Qin et al., 2006). To investigate possible mechanisms involved in determining phenotypic characteristics of type II activated macrophages, the role of the increase in IL-10 elicited by type II activation was investigated in order to elucidate whether it is responsible for some of the observed characteristics.

Classical and type II activated macrophages were cultured in the presence of either 5 $\mathrm{ng} / \mathrm{ml}$ recombinant IL-10 to enhance IL-10 levels, or $2 \mu \mathrm{g} / \mathrm{ml}$ anti-IL-10 antibody (ab) to block IL-10. Cytokine and cell surface markers were assessed 8 and $24 \mathrm{~h}$ post stimulation. Upon the addition of anti-IL-10 ab (Figure 3.5), no IL-10 was detected in any of the cultures indicating IL-10 has been successfully inhibited. Looking at IL-12 production, it can be seen that the inhibition of IL-10 led to an increase in IL-12 production by both classically and type II activated macrophages. In contrast, the addition of rIL-10 to cultures led to an almost complete inhibition of IL-12 production with only very low levels of IL-12 detected in cultures with classical and type II macrophages. Importantly, the absence of IL-10 did not impair the ability of type II activated macrophages to reduce IL12 in comparison to classically activated macrophages.

MCP-1 and IL-6 production were also assessed in response to IL-10 inhibition with anti-IL$10 \mathrm{ab}$, although not in response to the addition of IL-10. In the absence of IL-10, type II activation maintained reduced MCP-1. However the \% reduction was not as significant as seen under normal conditions. Both classically and type II activated macrophages increased MCP-1 production in the absence of IL-10, indicating IL-10 may cause some suppression of MCP-1 either directly or through the alterations of other important cytokines. While IL-6 was only assessed for one experiment, it appeared that IL-6 production was unaffected by the blockage of IL-10.

We wished to assess whether the increased IL-10 by type II activation was essential for the observed reductions in CD40 and CD80 expression. Contrary to expectations, the blockage of IL-10 had very little effect on CD40 or CD80 expression by both classically and type II activated macrophages. Moreover, the addition of rlL-10 to cultures also had no effect on the expression of these co-stimulatory markers with classical and type II macrophages exhibiting the same response seen under normal circumstances. As a 
whole, these data indicate that IL-10 alone does not play an essential role in regulating the cytokine and co-stimulatory marker profile elicited by type II activation. 


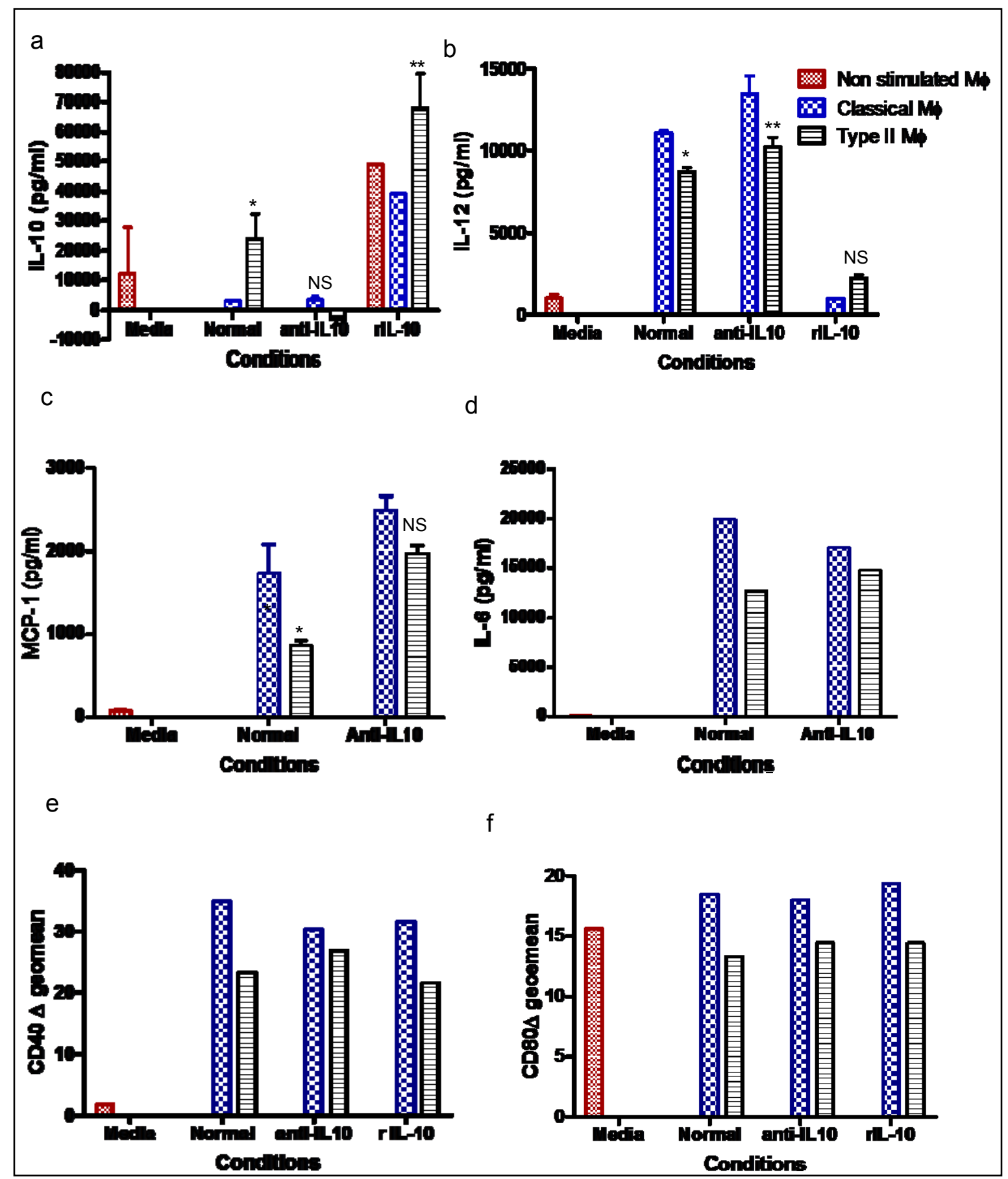

Figure 3.5 IL-10 inhibition does not alter the phenotype of type II activated macrophages. Bone marrow derived macrophages derived from C57BL/6 mice were exposed to classical and type IIinducing stimuli (Figure 3.3), with the addition of anti-IL-10 antibody $(2 \mu \mathrm{g} / \mathrm{ml})$ or recombinant IL-10 (5 ng/ml), or rat lgG as an isotype control ( $5 \mathrm{ng} / \mathrm{ml}$ ). After $8 \mathrm{~h}$ culture IL-10 (a), IL-12 (b), MCP-1 (c) and IL-6 (d) were assessed via ELISA. ${ }^{*} \mathrm{P}<0.05{ }^{* *} \mathrm{P}<0.01$ : Two way ANOVA with Bonferroni post test; Effects of classical versus type II activation under Normal conditions or IL-10 blockage. Data points (a-d) represent mean +SEM from duplicate wells. Data points in (d) represent single wells. After $24 \mathrm{~h}$ culture CD40 (e) and CD80 (f) were assessed via flow cytometry. Data points represent a single experiment. 


\subsubsection{Involvement of CD40 signalling in changes to the type II macrophage profile}

CD40 ligation induces the up-regulation of inflammatory cytokines such as IL-6 and IL-12, as well as co-stimulatory markers including CD80 (Alderson et al., 1993; Caux et al., 1994a). Studies have shown low levels of CD40 signaling induce IL-10 and inhibit IL-12 production, while strong signaling does the opposite in altering the cytokine environment to drive pro-inflammatory responses (Mathur et al., 2004). Our studies have shown that type II activated macrophages elicit an anti-inflammatory profile with significantly lower expression of CD40 compared to classically activated macrophages. Thus, we wished to determine the effect of the reduced CD40 expression on the anti-inflammatory profile by looking particularly at whether low levels of CD40 signalling were involved in perpetuating the anti-inflammatory phenotype of type II macrophages.

An agonistic anti-CD40 antibody $(8 \mu \mathrm{g} / \mathrm{ml})$ was added to cultures of classical and type II activated macrophages, and IL-12 and IL-10 levels were assessed 8 and $24 \mathrm{~h}$ following primary stimulation. Figure 3.6 illustrates the effects of additional CD40 stimulation on the expression of IL-12 and IL-10. IL-12 production by both classical and type II activated macrophages increased slightly upon additional CD40 stimulation. Addition of the isotype control had no effect on IL-12 or IL-10 production, indicating the effects were not due to non-specific binding. Although CD40 stimulation enhanced IL-12 production, the levels produced by type II activated macrophages in comparison to classical macrophages was still significantly reduced. Furthermore, IL-10 production by type II activation remained higher than levels produced by classical activation, both under normal circumstances and with the addition of the CD40 stimulatory antibody suggesting that the stimulation through CD40 did not shift the activation state of the macrophage from type II to classical or vice versa.

To identify the effects of altered CD40 signalling on the expression of co-signalling markers, CD80, MHC II and CD40 levels were assessed via flow cytometry. The expression of CD80 on type II activated macrophages remained unaltered by the addition of the stimulatory CD40 antibody, as did MHC II expression (Figure 3.6). CD40 expression on classical and type II activated macrophages was significantly reduced after the addition of stimulatory CD40 antibody. However, this reduction was most likely due to the agonistic stimulatory antibody preventing binding of the fluorescently labelled CD40 antibody used for flow cytometry. Irrespective of this, it can be seen that the levels of CD40 on type II activated macrophages remain lower than levels on classical macrophages, indicating enhanced CD40 signalling did not ablate the ability of type II activation to reduce this 
expression. However, because the data presented in Figure 3.6 is from one experiment, careful considerations must be made in drawing strong conclusions before confirmation of the findings. Together, these experiments indicate that CD40 signalling in type II activated macrophages has very little effect on the anti-inflammatory profile, despite the lower levels of CD40 expression. This suggests the macrophage phenotype occurs via a different pathway other than through altered CD40 signalling in the macrophage. This conclusion does not however exclude the possible role of reduced CD40 expression and thus signalling, through CD40L on T cells in response to type II activated macrophages, and requires further investigation. 


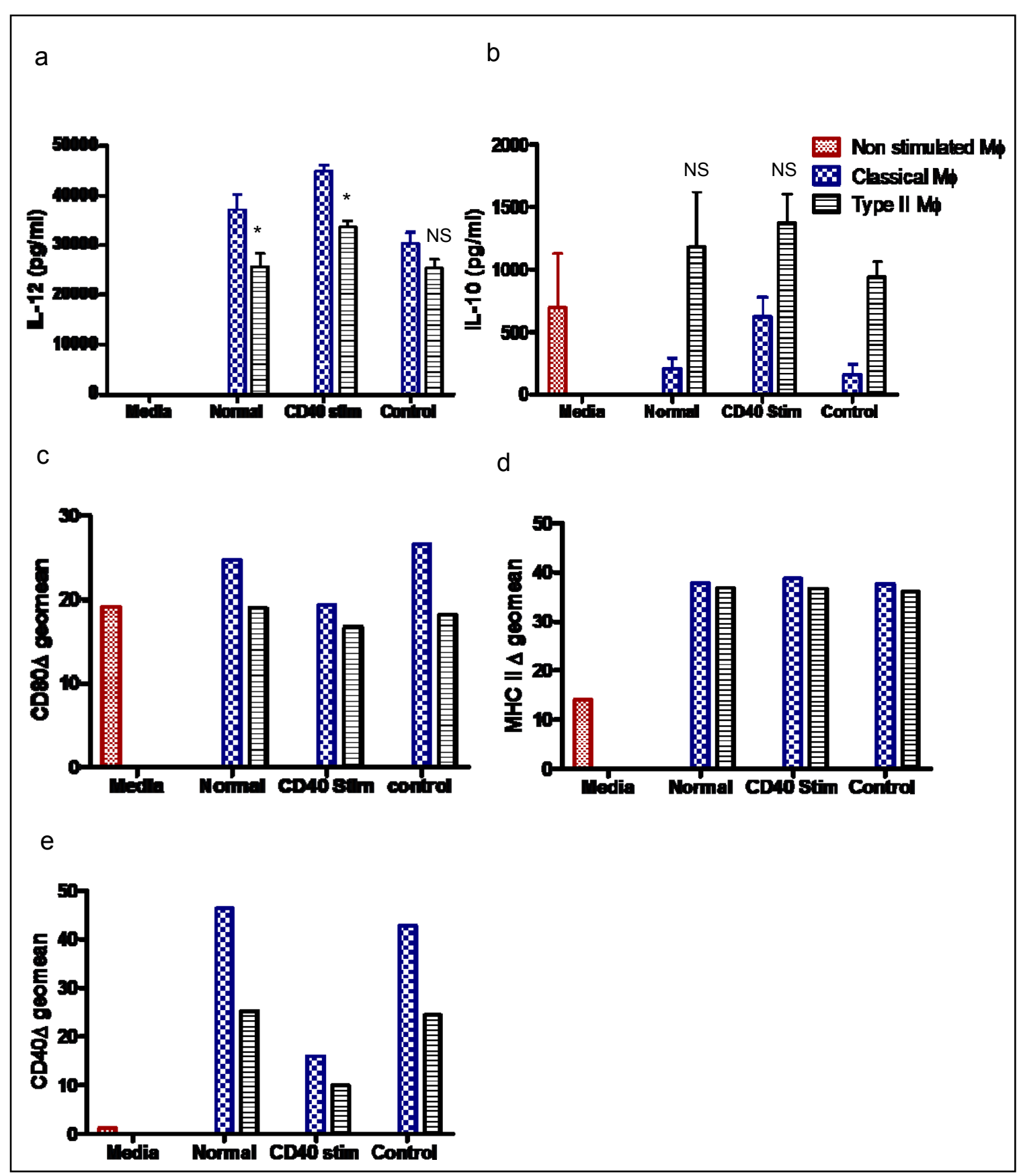

Figure 3.6 CD40 stimulation does not alter the phenotype of type II activated macrophages. Macrophages derived from C57BL/6 mice were exposed to classical and type II-inducing stimuli (Figure 3.3), with the addition of $8 \mu \mathrm{g} / \mathrm{ml}$ stimulatory rat-anti-mouse CD40 antibody (3/23) and 8 $\mu \mathrm{g} / \mathrm{ml} \mathrm{IgG}$ isotype control. After $8 \mathrm{~h}$ culture, IL-12 (a) and IL-10 (b) levels were accessed via ELISA. ${ }^{*} \mathrm{P}<0.001$ : Two way ANOVA with Bonferroni post test; Type II versus classical before and after CD40 stimulation. Bars represent duplicates from one experiment. After $24 \mathrm{~h}$ culture, CD80 (c), MHC II (d) and CD40 (e) were assessed via flow cytometry. Bars represent one experiment. 


\subsection{Discussion}

Type II activation deviates immune responses in a manner which significantly prevents the onset of inflammatory diseases (Gerber et al., 2001; Tierney et al., 2009). The mechanisms involved in type II activation are currently unknown, and full characterisation of the type II macrophage phenotype has yet to be elucidated. Therefore, this thesis investigated the effects of type II activation on a broader range of characteristics including cytokines, chemokines and surface markers. Until 2006, many researchers relied heavily on the IL-10/IL-12 ratio, and the Th2 biasing effect to identify type II activation (Anderson et al., 2002; Lucas et al., 2005; Sutterwala et al., 1997). Although there has been the identification of additional biochemical markers including enzymes and surface markers, the characterisation of this activation state is still limited. Therefore this thesis investigates the effects of type II activation on an array of markers to add to our existing repertoire of cytokines and surface markers altered by type II activation, while utilizing enzymatic profiling for further validation of this activation state.

In order to verify that our system induced type II macrophage activation, both the cytokine and enzymatic profile of primary macrophages stimulated classically with LPS was compared to those stimulated with opsonised SRBC in the presence of LPS. Classically activated macrophages stimulated with LPS, induced high production of IL-12, IL-6 and MCP-1 with little IL-10, in accordance with previous studies (Mantovani et al., 2004; Mosser et al., 2008). A more anti-inflammatory cytokine profile was elicited by exposure to LPS in the presence of opsonised SRBC. As seen previously seen by Gerber et al and Lucas et al, FcyR ligation via immune complexes in the presence of LPS suppressed the production of the pro-inflammatory cytokine IL-12, in parallel to increased production of the anti-inflammatory cytokine IL-10. The increase in IL-10 was specific to the ligation of Fc $\gamma$ Rs in the presence of TLR signalling via LPS, as no significant IL-10 production is seen in the presence of Fc $\gamma R$ ligation alone (opsonised SRBC without LPS). These results support previous observations (Lucas et al., 2005; Zhang et al., 2006), which demonstrated the requirement for the two signals for IL-10 up-regulation.

We also investigated the effect of $\mathrm{Fc} \gamma \mathrm{R}$ ligation on the response of macrophages to the production of other cytokines/mediators. We found that as with IL-12p40, Fc $\gamma R$ ligation reduced the production of MCP-1 and IL-6 in response to LPS. The effect of type II activation on chemokine production has not been previously reported, however the effects seen on IL-6 somewhat contradict previous literature (Gerber et al., 2001; Grazia Cappiello et al., 2001). Capiello et al reported the reduction in pro-inflammatory cytokines was specific to IL-12, and not IL-6, TNFa, or IL-1 $\beta$. However this conclusion came from the expression of IL-6 mRNA, not IL-6 protein secretion which we show here. It is possible 
that although IL-6 mRNA is unaffected (Grazia Cappiello et al., 2001), repressive effects may be occurring at the post transcriptional stages leading to reduced IL-6 secretion in response to type II activation. Additional work is required to verify whether this hypothesis is correct.

Th2 responses are associated with protection against EAE, through the counter-regulation of inflammatory immune responses (Cua et al., 1995; Kennedy et al., 1992; Shaw et al., 1997). Although type II activation increases the production of the prototypic Th2 cytokine IL-4 by CD4 T cells (Anderson et al., 2002; Tierney et al., 2009), it was unknown whether macrophages themselves were a contributor to Th2 development through macrophagederived IL-4 production. IL-4 has often been regarded as a T cell-derived cytokine with important roles in B cell proliferation/antibody production, as well as the commitment of $\mathrm{T}$ cells towards a Th2 subset via STAT-6 signalling (Kaplan et al., 1996; Le Gros et al., 1990). Until recently, it was not believed that macrophages could be a major source of IL4. However, IL-4 production in alveolar macrophages and macrophage cell lines have been reported (Buttner et al., 1997; Mukherjee et al., 2009; Pouliot et al., 2009), leaving open the question of whether type II macrophages can produce IL-4. Macrophages treated with LPS plus opsonised SRBC produced levels of IL-4 slightly greater than that of LPS treated macrophages; however, this increase did not reach significance suggesting that in this system, IL-4 is not produced to any great extent. Previous literature has reported the production of IL-4 by lung resident macrophages (Buttner et al., 1997), in the course of fibrosis induced by irradiation. However it was not fully understood how this occurred and what the implications were. Other than IL-4 production by alveolar macrophages and the RAW264.7 cell line, no other literature to date has fully examined the ability of macrophages to produce IL-4. Therefore, the appearance of IL-4 production, albeit at a low level, by the macrophages stimulated with LPS plus opsonised SRBC, indicates the possibility of type II macrophages producing IL-4. As IL-4 is largely involved in Th2 responses and protection against EAE (Le Gros et al., 1990; Shaw et al., 1997), further investigations into the effects of type II macrophages in IL-4 production and IL-4 responsiveness is warranted, especially with regards to IL-10 production which has been shown to up-regulate IL-4 receptor expression (Lang et al., 2002; Rückerl et al., 2006), as type II macrophages are very strong inducers of IL-10.

MCP-1 (monocyte chemotactic protein-1) is a chemokine involved in the chemotaxis of inflammatory cells such as monocytes, macrophages, and lymphocytes, and exacerbates inflammatory responses which are detrimental in the context of allograft rejection or EAE (Huang et al., 2001a; Lee et al., 2003; O'Shea et al., 2008). IL-6 is a pro-inflammatory cytokine which, when in the presence of TGF $\beta$, promotes the development of Th17 cells which are highly pathogenic during EAE (Langrish et al., 2005; Perona-Wright et al., 2009; 
Serada et al., 2008). High levels of IL-6, IL-12, and MCP-1 are associated with the peak of disease during EAE or MS (Maimone et al., 1997; McManus et al., 1998). Importantly, mice lacking MCP-1 are protected against EAE, associated with a lack of macrophage recruitment to the CNS, required for the induction of inflammatory Th1 responses (Huang et al., 2001a). Furthermore, inhibition of IL-6 protected mice against EAE through inhibition of Th17 development (Okuda et al., 1998; Serada et al., 2008). IL-12p40, through either IL-12p70 (IL-12p40/p35) or IL-23 (IL-12p40/p19) (Oppmann et al., 2000), also contributes to disease, through its induction of pathogenic Th1 or Th17 cells respectively (Becher et al., 2002; Cua et al., 2003). Reductions in IL-12-producing macrophages in the CNS and inhibition of IL-23 protect mice from developing EAE (Cua et al., 2003; Sewell et al., 2003). Therefore, the reduction in these pro-inflammatory mediators (IL-12p40, IL-6 and MCP-1), may be a mechanism which contributes to the protection type II activated macrophages elicit against inflammatory illnesses (Gerber et al., 2001; Tierney et al., 2009). Understanding mechanisms involved in perpetuating antiinflammatory responses by type II activated macrophages is therefore key to better understanding how to treat MS.

Edwards et al (2006) identified the differential expression of the enzyme sphingosine kinase 1 (SPHK1), specifically up-regulated in type II activated macrophages. We therefore underwent enzymatic profiling, comparing the effects of LPS to that of opsonised SRBC plus LPS on the production of iNOS and SPHK1. Figure 3.2 demonstrated the high expression of SPHK1 in cells stimulated with opsonised SRBC in the presence of LPS in comparison to LPS alone. Furthermore, iNOS was produced by macrophages in response to LPS, as well as LPS + opsonised SRBC, with similar levels of expression. These enzymatic profiles correlate to the enzymatic profile of type II activation, previously reported (Edwards et al., 2006). Therefore both the cytokine profile and enzymatic profile from our experiments correlate with previous studies, validating the successful induction of type II macrophage activation. How these enzymes contribute to the effector functions of type II macrophages is not yet known, but given the unique profile, further work is merited.

Although it was observed that type II activation significantly alters the cytokine profile away from inflammatory responses, it was unknown which processes contributed to the anti-inflammatory phenotype. To address this, changes in cytokine and surface marker expression on type II activated macrophages were assessed over a period of time after stimulation. As IL-10 and IL-12 production show the most distinctive alterations in response to type II activation, kinetic analyses were carried out on these two cytokines in order to identify which time point the most notable changes occurred. Over a period of time from 2-24 h, type II activation maintains reduced levels of IL-12 compared to classical 
macrophages, with no significant changes in the \% difference over time. However, there is a significant difference in the \% increase in IL-10 over time. Type II activated macrophages exhibit the most significant increase in IL-10 at $8 \mathrm{~h}$ post-stimulation, illustrating the optimal time point of $8 \mathrm{~h}$ for assessing the immune-modulating effects of type II activation on cytokine production.

In addition to cytokines, co-signalling molecules are also important for regulating the type of immune response which follows (Lenschow et al., 1996). The effects of type II macrophage activation on the expression of co-signalling markers CD40, CD80, PDL-1 and $\mathrm{MHC}$ II was assessed via flow cytometry. As with IL-12, expression of these surface markers are up-regulated on macrophages stimulated classically with LPS and IFN $\gamma$ (Edwards et al., 2006; Qin et al., 2006; Tierney et al., 2009). In correlation with these observations, data presented in this thesis also show classically activated macrophages up-regulate these surface markers. Compared to the media controls, the increased expression of MHC II on LPS-stimulated macrophages correlates with observations by Edwards et al (2006). Levels of MHC II are up-regulated on antigen presenting cells such as macrophages in response to the prototypic cytokine IFN $\gamma$, and are an indicator of the ability of macrophages to induce CD4 T cell responses (Edwards et al., 2006; O'Keefe et al., 2001). At the $8 \mathrm{~h}$ time point, there is very little difference seen in the levels on type II activated macrophages compared to classical macrophages, as also demonstrated by Tierney et al (2009). However, this effect changes with time, as type II activation elicits reductions in MHC II in response to LPS at 24-30 h. These results contrast previous studies, which indicated MHC II levels for type II activated macrophages increased $24 \mathrm{~h}$ post stimulation (Edwards et al., 2006). This difference may be due to slight differences in the experimental setting under which type II activation was induced, leading to differences in the timing of certain responses. Also, as this data on $\mathrm{MHC}$ II is from a single experiment, repeats are required to confirm this observation. Aberrant MHC II expression is associated with the induction of autoimmunities such as MS, where certain MHC II haplotypes are associated with increased risk of developing multiple sclerosis (MS) (Dyment et al., 2005). High levels of MHC II are expressed in the CNS during inflammatory disorders such as EAE, and Alzheimer's, implicating their role in inflammatory immune responses (Baranzini et al., 2000; Perlmutter et al., 1992). In mice immunized to exhibit EAE, a deficiency in the transactivator of $\mathrm{MHC}$ II expression, which is involved in regulating the expression of inducible $\mathrm{MHC}$ II genes, resulted in protection from disease (Stuve et al., 2002). This demonstrates the possible benefits of type II macrophages reducing their expression of $\mathrm{MHC} \mathrm{II}$ in the context of preventing inflammation. 
The expression of a co-stimulatory molecule CD80 was also assessed. CD80 is an important co-stimulatory molecule involved in $T$ cell responses. Through the binding of CD80 to CD28 or CTLA-4 on T cells, CD80 is able to promote or inhibit the activation of CD4 T cells in collaboration with MHC II (Alegre et al., 2001). Corresponding with previous observations by Tierney et al (2009), no significant difference in the expression of CD80 in media-treated or LPS-treated macrophages was seen 2, 4, 8, 24 and $30 \mathrm{~h}$ after stimulation. Again correlating with Tierney et al, type II activated macrophages exhibited a significant reduction in the expression of CD80. This reduction in CD80 expression could be observed not only at $8 \mathrm{~h}$ post stimulation (Tierney et al., 2009), but also at 2, 4, 24 and $30 \mathrm{~h}$ post stimulation. The most significant reduction in CD80 expression was observed 24 h after stimulation. CD80, as with CD40, is an important co-stimulatory molecule which binds to CD28 on T cells, and aids in determining the outcome of the macrophage-T cell interaction. CD80 and CD40 both play synergistic roles in the induction of proinflammatory responses, as demonstrated in a cecal ligation by puncture (CLP) model of polymicrobial sepsis where mice deficient in CD80 exhibited a reduced rate of mortality, accompanied by reduced pro-inflammatory cytokines (Nolan et al., 2009). On the other hand, wild type mice with CLP had high levels of CD80 accompanied by increased mortality. In patients with MS, increased levels of CD80 are seen at lesion sites (Windhagen et al., 1995), and in EAE, high levels of CD80 have been correlated with disease progression (Karandikar et al., 1998). The fact that the levels of CD80 and MHC II expression on type II activated macrophages are below that seen on classical macrophages indicates type II activation may be impairing the ability to drive $\mathrm{T}$ cell responses, and present antigen to $T$ cells. This could be beneficial in the context of autoimmunities whereby a reduction in the activation of autoreactive $T$ cells can prevent progression of disease.

PD-1 is a molecule present on T cells, which has a role in limiting $T$ cell responses and maintaining peripheral tolerance (Nishimura et al., 1999). PDL-1 is a ligand for PD-1, which is constitutively expressed on a variety of cells including macrophages, and is upregulated in response to inflammatory stimuli (Freeman et al., 2000; Yamazaki et al., 2002). We investigated PDL-1 expression on type II and classically activated macrophages. Previous experiments (Tierney et al., 2009) looking at an $8 \mathrm{~h}$ time point have demonstrated a significant reduction in PDL-1 by type II activation in comparison to classically activated macrophages. Correlating with Tierney et al, at $8 \mathrm{~h}$ post stimulation a reduction in PDL-1 on type II activated macrophages can be seen in comparison to classical activation. However, this reduction is minimal in comparison to the significant decrease exhibited 24 or $30 \mathrm{~h}$ post stimulation. This emphasizes the importance of looking at multiple time points when assessing the effects of type II activation on surface marker 
expression. PD-1, to which PDL-1 binds to, is associated with inhibition of $T$ cell responses through the inhibition of proliferation and cytokine production (Freeman et al., 2000; Latchman et al., 2001), and has been associated with the induction of peripheral tolerance where its absence has lead to the development of autoimmunites such as arthritis, lupus and cardiomyopathy (Nishimura et al., 1999; Nishimura et al., 2001). Due to the role of PD-1 ligation in inhibiting T cell proliferation, it was previously presumed type II activated macrophages which have reduced levels of the ligand for PD-1, would support $\mathrm{T}$ cell proliferation. However, in addition to inhibition of $\mathrm{T}$ cell proliferation, other effects of the PD-1 ligands have been noted, where their involvement in enhancing, rather than inhibiting $T$ cell responses has been observed (Tseng et al., 2001; Wang et al., 2003). Furthermore PDL-1 and PDL-2 have shown differential responses to Th1/Th2 cytokines (Loke et al., 2003). Therefore it is possible that the reduction in PDL-1 plays a role in inhibiting or promoting Th1/Th2 biasing. If PDL-1 was associated with Th1 biasing, as has been previously suggested as a possibility (Loke et al., 2003), a reduction in PDL-1 by type II activation may be beneficial during inflammatory disease. Studies are currently underway to try and further understand the complexity of the PDL-1/PD-1 interaction and their different functions.

CD40 is an important co-signalling molecule involved in the activation of Th1 cells (Campbell et al., 1996; Kelsall et al., 1996). At 24-30 h where CD40 is most highly expressed in response to LPS (Figure 3.4), type II activated macrophages exhibited the most significant reduction in the expression of this co-stimulatory molecule. The observation corresponds to that previously reported at an $8 \mathrm{~h}$ time point (Tierney et al., 2009). The level of CD40 on type II activated macrophages is above that seen on nonactivated macrophages, but significantly lower than levels on classically activated macrophages. CD40 is an essential co-stimulatory molecule for the induction of Agspecific T cell responses (Grewal et al., 1998). Although CD40 is associated with cellmediated inflammatory responses (Stout et al., 1996), studies have also shown in the absence of this co-stimulatory molecule, Th2 development is impaired (MacDonald et al., 2002b), illustrating the dual role of CD40 in promoting both Th1 and Th2 counter regulatory responses. CD40 ligation has also been attributed to increased production of inflammatory mediators and surface molecules such as CD80, which aids Th1 responses (Alderson et al., 1993; Caux et al., 1994b). Furthermore, CD40 promotes the development of pathogenic Th17 cells through the production of IL-6 (Perona-Wright et al., 2009). A reduction in CD40 on type II activated macrophages may therefore promote the suppression of inflammatory mediators, thus reducing inflammatory disease. Some studies have also shown the ability of CD40 to promote anti-inflammatory or proinflammatory responses depending on their level of signalling (Mathur et al., 2004; 
Murugaiyan et al., 2006). Therefore the particular low level of CD40 expressed on type II activated macrophages may be instrumental in preventing the development of inflammatory responses by Th1 and Th17 cells, while promoting anti-inflammatory Th2 responses, to provide protection against EAE. The role of CD40 requires further investigation, as does the contribution of the increased IL-10 by type II activation.

We investigated whether the increase in IL-10 is essential for the type II activation phenotype, which consists of a reduction in CD40,CD80, PDL-1 as well as decreased IL12 and MCP-1. Our finding that rIL-10 addition significantly decreases IL-12 production correlates with previous studies demonstrating a direct suppressive effect of IL-10 on IL12 (Schottelius et al., 1999). IL-12 production from cells including monocytes (de Waal Malefyt et al., 1991), the RAW264.7 cell line (Rahim et al., 2005), as well as primary macrophages (Anderson et al., 2002), are inhibited by IL-10. More importantly, in agreement with previous studies (Anderson et al., 2002), the reduction in IL-12 production observed with type II activation was in no way affected by the blockage of IL-10. The ability of type II activated macrophages to reduce the production of MCP-1 and IL-6 was also largely unaffected by the absence of IL-10, again implicating an independent mechanism involved in the reduction of inflammatory cytokines by type II activation. The mechanism whereby additional IL-10 is able to inhibit IL-12, yet IL-10 is not required for the inhibition of IL-12, IL-6 or MCP-1 by type II activation, is yet to be understood.

The role of IL-10 on alterations in surface markers by type II activation was also assessed. Previous literature reported a reduction in CD40 on macrophage cell lines in response to LPS plus IL-10 (Qin et al., 2006). However, this investigation found, as with the cytokine profile, reductions in the expression of co-stimulatory markers CD40 and CD80 was not dependant on IL-10, although the data on the expression of these surface markers is only from one experiment and must be interpreted with caution. However, these preliminary results are a good indicator that even though IL-10 and IL-12 alterations occur prior to changes in surface marker expression, these alterations do not solely direct reductions in the expression of surface markers and other pro-inflammatory cytokines. This indicates type II activation reduces inflammatory cytokines/surface markers through an alternative mechanism other than IL-10. It is necessary to identify these mechanisms, which may in fact be occurring in synergy with IL-10. Once these essential mechanisms are identified, it may be possible to enhance the ability of macrophages to promote anti-inflammatory responses.

Due to the dual role of CD40 previously mentioned (Mathur et al., 2004), we wished to investigate if CD40 stimulation was involved in the other phenotypic properties of type II activated macrophages. We hypothesized that this reduction in CD40 by type II activation 
causes a reduced level of signaling such that low levels induce the production of antiinflammatory cytokines and suppress pro-inflammatory responses. Previous studies have demonstrated low level signaling up-regulates IL-10 in comparison to high level signaling which promotes IL-12 production by DCs (Murugaiyan et al., 2006). To my knowledge, the dual role of CD40 signaling in primary macrophages from C57BL/6 mice has not been investigated. This investigation utilized an agonistic stimulatory antibody for CD40, in order to enhance signaling on type II activated macrophages. The theory being, that if the reduction in CD40 occurred with the goal to reduce signaling and elicit anti-inflammatory characteristics, by increasing CD40 signaling, the anti-inflammatory effect of type II activation will be impaired.

High levels of CD40 stimulation had no effect on the ability of type II activated macrophages to reduce the production of IL-12 and increase IL-10 in comparison to classically activated macrophages. These observations conflict those seen by Mathur et al (2004). Mathur et al demonstrated low levels of CD40 signalling with 2-4 $\mu \mathrm{g} / \mathrm{ml}$ of antiCD40 ab increased IL-10 in BALB/c derived peritoneal macrophages. We observed that at high levels of CD40 signalling, type II activated macrophages maintained higher levels of IL-10 and reduced IL-12 in comparison to classical macrophages. This indicates the increased production of IL-10 and reduced IL-12 occurred independently of any reductions in CD40 signalling by type II activation.

Previous studies have demonstrated enhanced expression of surface markers in response to CD40 signalling (Alderson et al., 1993). Data presented in this thesis demonstrates enhanced CD40 stimulation has no effect on the expression of CD80 or MHC II by both classically activated and type II activated macrophages. Furthermore, type II activated macrophages maintained reduced CD40 levels compared to classical macrophages, as seen under normal stimulation conditions. The significant reduction in CD40 detected by both macrophage subsets is most likely due to the CD40 stimulatory antibody remaining bound to CD40 present on macrophages. This preliminary data indicates low levels of CD40 signalling is not essential for the type II macrophage phenotype, as indicated by the effects of enhanced CD40 signalling. These observations correlate to the previously described lack of effect of IL-10 alterations on the phenotype induced by type II activation. However, one explanation for differences between Mathur et al and this data may be that the responses by bone marrow-derived C57BL/6 macrophages and peritoneal BALB/c macrophages differ in the specific levels of CD40 which define low level and high level signalling. This possibility requires further investigation. 
Although this thesis identified increased CD40 signalling did not impair the ability of type II activation to elicit an anti-inflammatory phenotype, identifying the effect of reducing CD40 expression or signalling on classical macrophages would provide more information on the importance of CD40 expression levels. This investigation identified additional effects of type II activation on cytokine/chemokine and surface marker expression in addition to illustrating that the anti-inflammatory profile occurs independently of IL-10. These findings expand our current knowledge of the type II activation state, and further emphasise the importance of understanding the mechanisms involved in promoting the phenotypic properties elicited by type II activation, as many of the characteristics are indicative of eliciting beneficial effects against inflammatory disease. 


\section{Chapter 4: Elucidating the effects of type II macrophages on $\mathrm{T}$ cell responses}

\subsection{Introduction}

T cell differentiation into Th1, Th2, or other subsets is largely determined by the cytokine environment under which the $\mathrm{T}$ cells are stimulated. Antigen presenting cells such as macrophages and dendritic cells which produce a variety of cytokines in response to different micro-environments play a major role in determining the outcome of $\mathrm{T}$ cell differentiation (Stein et al., 1992; Stout et al., 2004). Once differentiated, the subset functions are characterised by the cytokines the $T$ cells subsequently produce (Abbas et al., 1996; Wan et al., 2009). Th1 cells produce IFN $\gamma$ and support cell-mediated responses whereas Th2 cells can be characterised by their production of regulatory cytokines such as IL-13, IL-5, and IL-4 (Abbas et al., 1996; Wan et al., 2009). Strong promoters of Th1 development are IL-12, and Th2 development IL-4 and IL-2 (Cote-Sierra et al., 2004; Le Gros et al., 1990; Liao et al., 2008). In addition to cytokines in the environment, direct interaction between T cells and APCs through MHC:TCR and co-stimulatory molecules is essential in the development of different T cell subsets. Of particular interest is the costimulatory molecule CD40 ligand (CD40L) present on T cells and its' receptor CD40 on APCs (Grewal et al., 1998).

CD40 signalling exhibits dual roles. It has a well documented role in promoting cell mediated immunity and driving pro-inflammatory responses which are essential for the clearance of intracellular pathogens and anti-tumour responses (Campbell et al., 1996; DeKruyff et al., 1997; Florido et al., 2004; Stout et al., 1996). It has also been reported as an essential mechanism of Th2 development (MacDonald et al., 2002b). Benefits of blocking the Th1-promoting effects of CD40L signalling have been demonstrated in animal models of EAE (Girvin et al., 2000; Howard et al., 1999), yet in terms of Th2 development, the exact effects of CD40 signalling between $T$ cells and macrophages is less well understood. In the absence of CD40 or CD40L as seen in knockout mice, Th2 responses are inhibited (MacDonald et al., 2002a). Furthermore, studies have found that CD40 inhibition in vivo by administration of anti-CD40L antibodies, polarises immune responses from a Th1 dominant one into a Th2 biased response (Hancock et al., 1996). This dual role of CD40 may lie in the length (Lee et al., 2002), or strength of signal, where strong CD40 signalling induces Th1 dominant responses which are useful for inducing antitumour responses (Schoenberger et al., 1998), whereas low level signalling increases the production of regulatory cytokines such as IL-10 by APCs, which promote Th2 and antiinflammatory responses (Mathur et al., 2004; Murugaiyan et al., 2006). Much still remains 
to be investigated in order to understand the exact mechanisms which determine the ability of CD40/CD40L interactions to drive counter-regulating Th1/Th2 immune responses.

Type II activated macrophages are capable of biasing $T$ cell responses towards the development of Th2 cells (Anderson et al., 2002; Gerber et al., 2001), and mechanisms involved in this biasing need elucidation. The role of CD40 in the context of type II activation is unknown; however, recent studies have shown that type II activation reduces CD40 as well as PDL-1 expression on macrophages (Tierney et al., 2009). The ability of type II activated macrophages to alter adaptive immune responses has beneficial consequences in the protection from inflammatory illnesses such as endotoxemia (Gerber et al., 2001) and EAE (Tierney et al., 2009), and the type of interaction which occurs between $T$ cells and macrophages can determine whether a protective Th2 response occurs (Cua et al., 1995), or pathology-inducing Th1/Th17 response ensues (Becher et al., 2002; Langrish et al., 2005).Therefore, it is important to understand the different mechanisms involved in regulating the outcome of $\mathrm{T}$ cell subset differentiation.

This thesis investigates the mechanisms involved in Th2 biasing by type II activated macrophages with the long term goal of understanding mechanisms that may lead to protection against pro-inflammatory diseases.

Aims:

The ability of type II activated macrophages to induce Th2 cell biasing has previously been reported (Anderson et al., 2002; Gerber et al., 2001), and has been shown to promote protection from EAE (Tierney et al., 2009). However, the mechanisms by which type II activated macrophages drive such responses are still unclear. Both Th1 and Th2 responses have demonstrated a dependency of CD40/CD40L signalling (Campbell et al., 1996; MacDonald et al., 2002a). Other studies demonstrate enhanced Th2 development by CD40L inhibition (Hancock et al., 1996). These contradictory observations may be due to differences in the level of CD40 inhibition, whereby a particular low level of inhibition elicits Th2 polarisation, which is otherwise inhibited in the complete absence of CD40L. This idea is supported by studies that show different levels of CD40 signalling on dendritic cells and macrophage cell lines, promote counter-regulatory Th1/Th2 responses both in vitro and in vivo (Mathur et al., 2004; Murugaiyan et al., 2006).

As discussed in chapter 3, type II activated macrophages exhibit significant reductions in the expression of CD40 and PDL-1, while still being a potent inducer of Th2 responses (Anderson et al., 2002). The goal of this thesis was to investigate mechanisms involved in $\mathrm{T}$ cell biasing by type II activated macrophages. To further understand mechanisms 
involved in promoting Th2 development, we examined how altering the level of signalling between CD40 and CD40L influences Th ( $T$ helper) cell subset differentiation. In particular, we addressed three specific aims:

1. To compare the effects of type II activation and classical activation on $\mathrm{T}$ cell proliferation and activation, given the reduced expression of PDL-1 on type II macrophages.

2. To determine if low levels of CD40 signalling promote the generation of Th2 cells.

3. To investigate whether Th2 cell biasing can be enhanced by reducing the CD40/CD40L interaction. 


\subsection{Results}

\subsubsection{Effects type II activation has on CD4 T cell proliferation}

In Chapter 3, the difference in the phenotype of type II and classically activated macrophages was investigated. As with previous studies (Tierney et al., 2009), a significant reduction in the expression of several co-stimulatory molecules including CD40 and PDL-1 expression was observed on type II activated macrophages. PDL-1 has been associated with the induction of T cell anergy, as well as T cell stimulation (Keir et al., 2006; Latchman et al., 2001; Tseng et al., 2001). Given that a reduction in PDL-1 would be expected to enhance $T$ cell proliferation, investigations were carried out to assess if $T$ cell proliferation was altered in our experimental system, given the observed reduction in PDL-1 on type II activated macrophages.

To investigate $T$ cell subset differentiation, we used naïve $T$ cells isolated from OTII mice whose $T$ cells express a transgenic TCR specific for $\mathrm{OVA}_{323-339}$ peptide in the context of MHC II. Macrophages were non-activated or were classically or type II activated as previously described (sec 2.2). Purified CD4 OTII cells were stained with carboxyfluorescein diacetate succinimidyl ester (CFSE) and added to the macrophage cultures 2-3 $\mathrm{h}$ post-stimulation, either in the absence or presence of OVA protein or $\mathrm{OVA}_{323-339}$ peptide. $72 \mathrm{~h}$ following co-culture, T cells were removed and assessed by flow cytometry for their degree of proliferation as determined by CFSE dilution. Because CFSE is a cytoplasmic dye which is split evenly between daughter cells upon mitosis, the reduction in the fluorescence of cells correlates to the level of proliferation that has occurred (Lyons et al., 1994).

All macrophages were able to present antigen and activate T cell proliferation (Figure 4.1). $\mathrm{T}$ cells exposed to either classical or type II activated macrophages presenting OVA protein or $\mathrm{OVA}_{323-339}$ peptide exhibited similar levels of antigen-specific proliferation (Figure 4.1). The response to the different antigen forms were similar, except that $\mathrm{OVA}_{323-}$ 339 peptide was more antigenic and induced greater $\mathrm{T}$ cell proliferation. $\mathrm{T}$ cells exposed to non-stimulated macrophages presenting $\mathrm{OVA}_{323-339}$ peptide had similar levels of antigenspecific proliferation as those induced by classical and type II activation; however, no proliferation was exhibited in response to non-stimulated macrophages that presented OVA protein. Only background proliferation was detected in co-cultures of $T$ cells and macrophages containing only medium. Therefore, $\mathrm{T}$ cell proliferation in response to antigen presented by macrophages was similar irrespective of whether they were classical or type II activated. 
IL-2 cytokine production was also assessed (Figure 4.2a), as another indicator of a T cell activation. T cells cultured alone did not produce significant amounts of IL-2, nor did T cells cultured with macrophages in the absence of antigen. In contrast, T cells exposed to macrophages in the presence of antigen, showed significant production of IL-2, and T cells cultured in the presence of non-stimulated macrophages had the highest amounts of IL-2 detected in their supernatant. In comparison, $\mathrm{T}$ cells exposed to classical macrophages showed lower levels of IL-2, and T cells cultured with type II activated macrophages had the lowest levels of antigen-specific IL-2 detected in the supernatant. These results may suggest that less IL-2 is produced by $T$ cells stimulated by type II activated macrophages; however, increased reuptake of IL-2 may be another explanation. This possibility was further explored by assessing the expression of IL-2Ra on T cells in these cultures. Interestingly the expression of this high affinity IL-2 receptor is increased and may account for increased reuptake of IL-2 (Figure $4.2 b$ ).

Overall, this data suggests type II activated macrophages elicit similar proliferative responses by CD4 $\mathrm{T}$ cells to that induced by classically activated macrophages. 


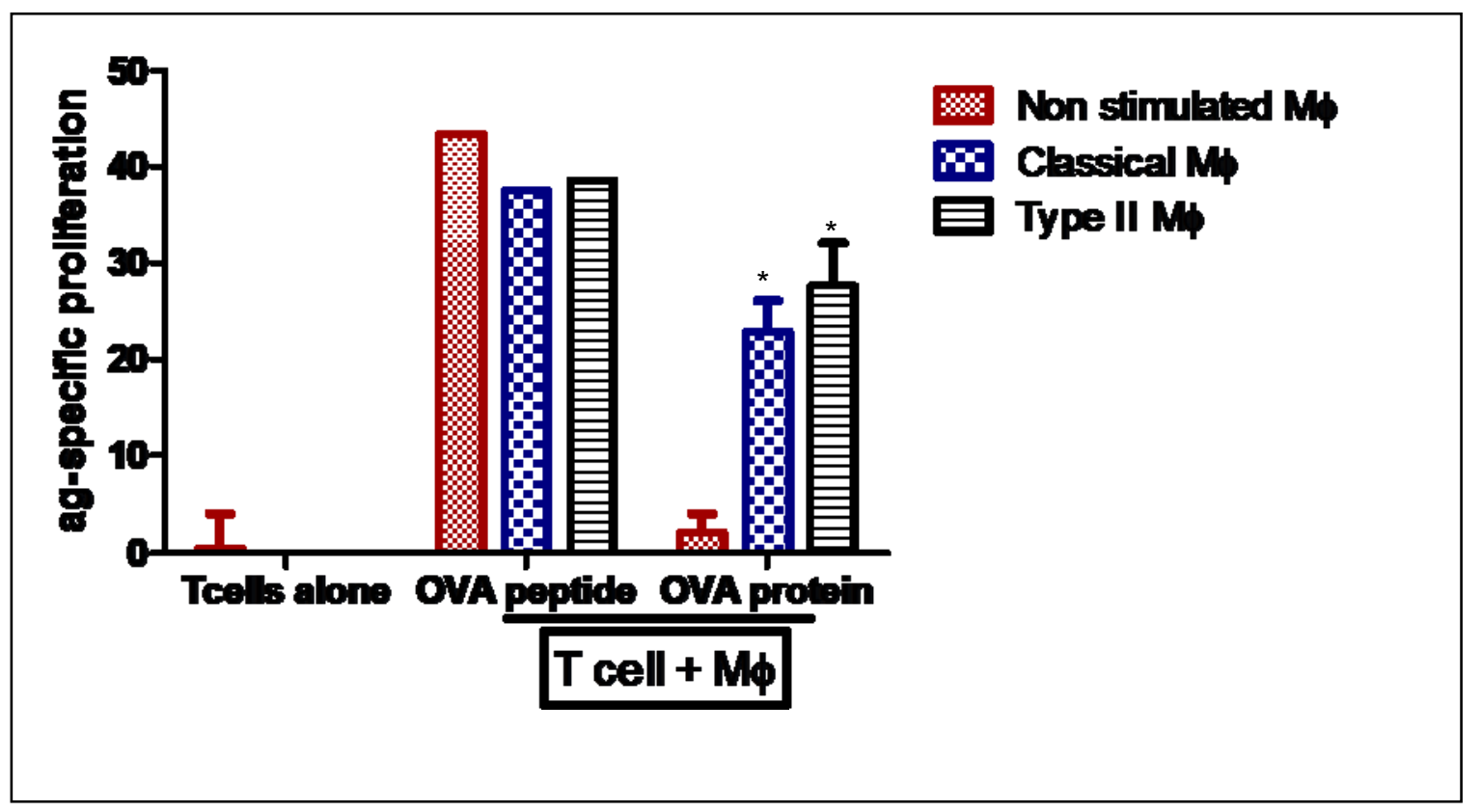

Figure 4.1 Type II and classical activation support similar levels of antigen-specific $\mathrm{T}$ cell proliferation. Macrophages $\left(10^{6} \mathrm{cells} / \mathrm{ml}\right)$ were exposed to classical or type II activating stimuli. CD4 T cells were isolated from OTII mice (sec 2.2) and stained with CFSE. CD4 T cells $\left(2.5 \times 10^{6}\right.$ cells $/ \mathrm{ml}$ ) plus $1 \mu \mathrm{M} \mathrm{OVA}_{323-339}$ peptide or $150 \mu \mathrm{g} / \mathrm{ml}$ OVA protein were added to macrophage cultures $3 \mathrm{~h}$ after classical or type II activation. After $72 \mathrm{~h}$ culture, CFSE was assessed by flow cytometry and presented as relative proliferation compared to cultures without antigen. The data from two experiments is shown. Note: Bars for 'OVA peptide' represent one experiment. ${ }^{*} \mathrm{P}<0.05$ : Two way ANOVA with Bonferroni post-test; Responses to macrophage states with antigen present versus antigen absent. Bars represent data points (mean+SEM) of two experiments. Relative proliferation $=\%$ decrease in CFSE of co-culture conditions in the presence of antigen relative to conditions in the absence of antigen. 


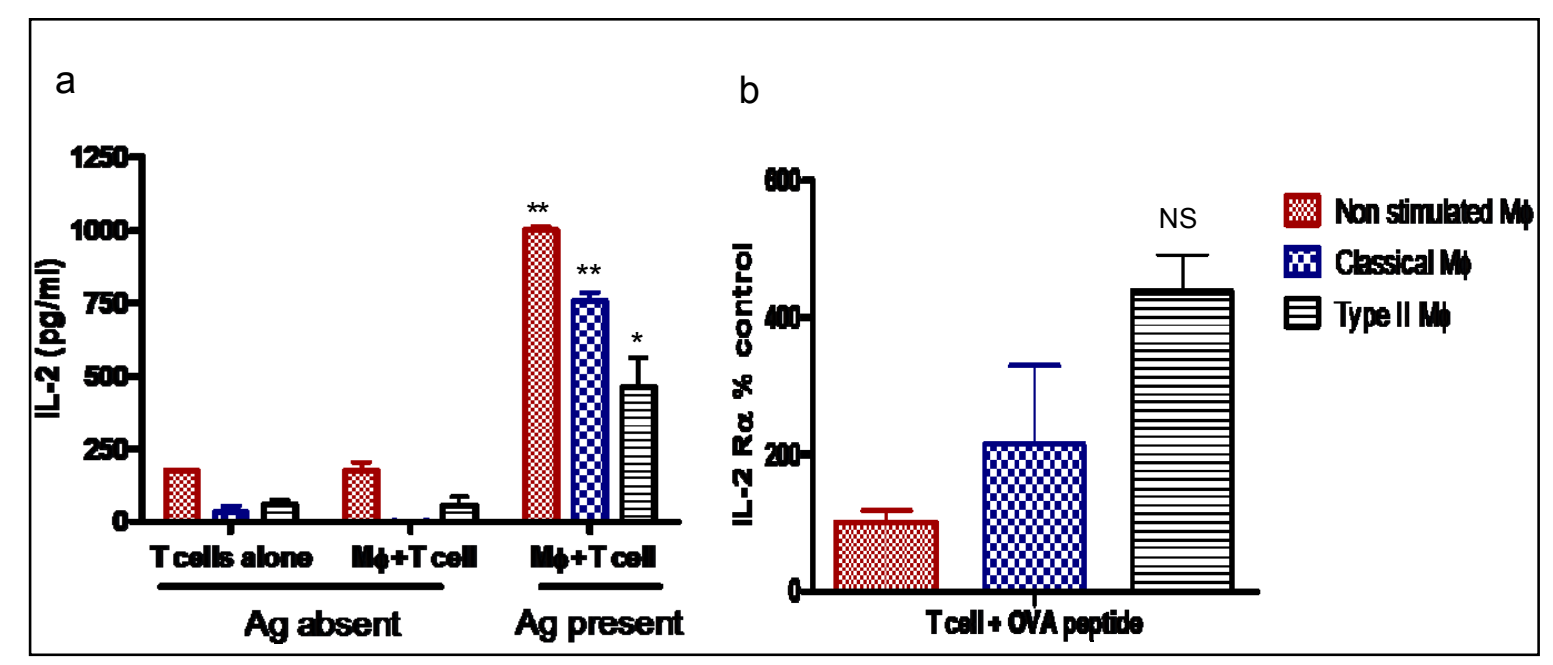

Figure 4.2 Type II and classical activation induce different levels of antigen-specific IL-2 production. Macrophages $\left(10^{6} \mathrm{cells} / \mathrm{ml}\right)$ were co-cultured with CD4 T cells $\left(2.5 \times 10^{6} \mathrm{cell} / \mathrm{ml}\right)$ as described in Figure 4.1. After $72 \mathrm{~h}$ culture, IL-2 was assessed via ELISA (a), and IL-2R $\alpha$ was assessed via flow cytometry (b), and presented as \% of control compared to cultures with nonactivated macrophages. The data from two experiments is shown. ${ }^{*} P<0.01$, ${ }^{* *} P<0.001$ : Two way ANOVA with Bonferroni post-test; (a) Responses to macrophage states with antigen present vs antigen absent. (b) P> 0.05: One way ANOVA; responses to type II versus classical MØ. Bars represent data points (mean+SEM) from duplicate wells of two experiments. 


\subsubsection{Effect of type II activation on CD4 T cell biasing}

Previous studies have identified the ability of type II activated macrophages to alter adaptive immune responses through the polarisation of a pro-inflammatory Th1 response into a Th2 dominant response (Anderson et al., 2002; Gerber et al., 2001; La Flamme et al., 2006; Tierney et al., 2009). In order to identify ways of enhancing Th2 biasing, we first needed to understand the effects of type II activated macrophages on naive CD4 T cells in the context of our experimental system. To this end, naïve CD4 T cells from OTII mice were cultured with either non-stimulated, classically activated or type II activated macrophages in the presence of $\mathrm{OVA}_{323-339}$ peptide. Cultures were maintained for $72 \mathrm{~h}$, after which IFN $\gamma$ and IL-4 were analysed via ELISA and IL-4Ra and IL-2Ra (CD25) expression were assessed via flow cytometry.

Figure 4.3 demonstrates that similar levels of IFN $\gamma$ were detected by T cells cultured with non-stimulated or classically activated macrophages (a). In comparison, IFN $\gamma$ production by CD4 T cells exposed to type II activated macrophages was significantly reduced, indicating a biasing against differentiation into IFN $\gamma$-producing Th1 cells. Although IL-4 production is the best marker of Th2 cells, only very low levels of IL-4 were detected in the cultures and no difference between the presence of the different macrophage activation states was found. These results confirm that classically activated macrophages and not type II activated macrophages promote Th1 differentiation.

As another measure of Th2 biasing, we investigated the responsiveness of T cells to IL-4 by measuring the expression level of its receptor IL-4Ra (b). T cells exposed to nonstimulated and classically activated macrophages had much lower levels of IL-4Ra expression in comparison to those exposed to type II activated macrophages, suggesting that type II macrophages induce T cells to become more responsive to this prototypic Th2 cytokine. Additionally, IL-2Ra was highly expressed on T cells exposed to type II activated macrophages (Figure 4.2). While IL-2Ra expression on $\mathrm{T}$ cells was also up-regulated in response to classically activated macrophages, the levels were below those of $T$ cells exposed to type II activated macrophages. These results indicate that type II activation increases CD4 $T$ cell responsiveness to IL-4 and IL-2 and thus may promote the differentiation of these cells to the Th2 phenotype.

As previously seen in other systems (Anderson et al., 2002; Edwards et al., 2006; Gerber et al., 2001), the findings presented here indicate type II activated macrophages are capable of inducing Th2 cell biasing as identified by increased responsiveness to IL-4Ra and IL-2Ra as well as reduced production of IFN $\gamma$. Investigations are still underway to identify the exact role of IL-2 in Th1/Th2 biasing, but our results further support the notion that IL-2Ra expression in addition to other factors such as IL-4Ra, have an active role in 
inducing Th2 biasing (Cote-Sierra et al., 2004; Liao et al., 2008). As IL-4 cytokine production cannot be reliably detected due to the low levels at the time points tested, the use of IL-2Ra expression in conjunction with other markers makes it possible to carry out further experiments elucidating mechanisms involved in $\mathrm{T}$ cell biasing by type II activated macrophages. 


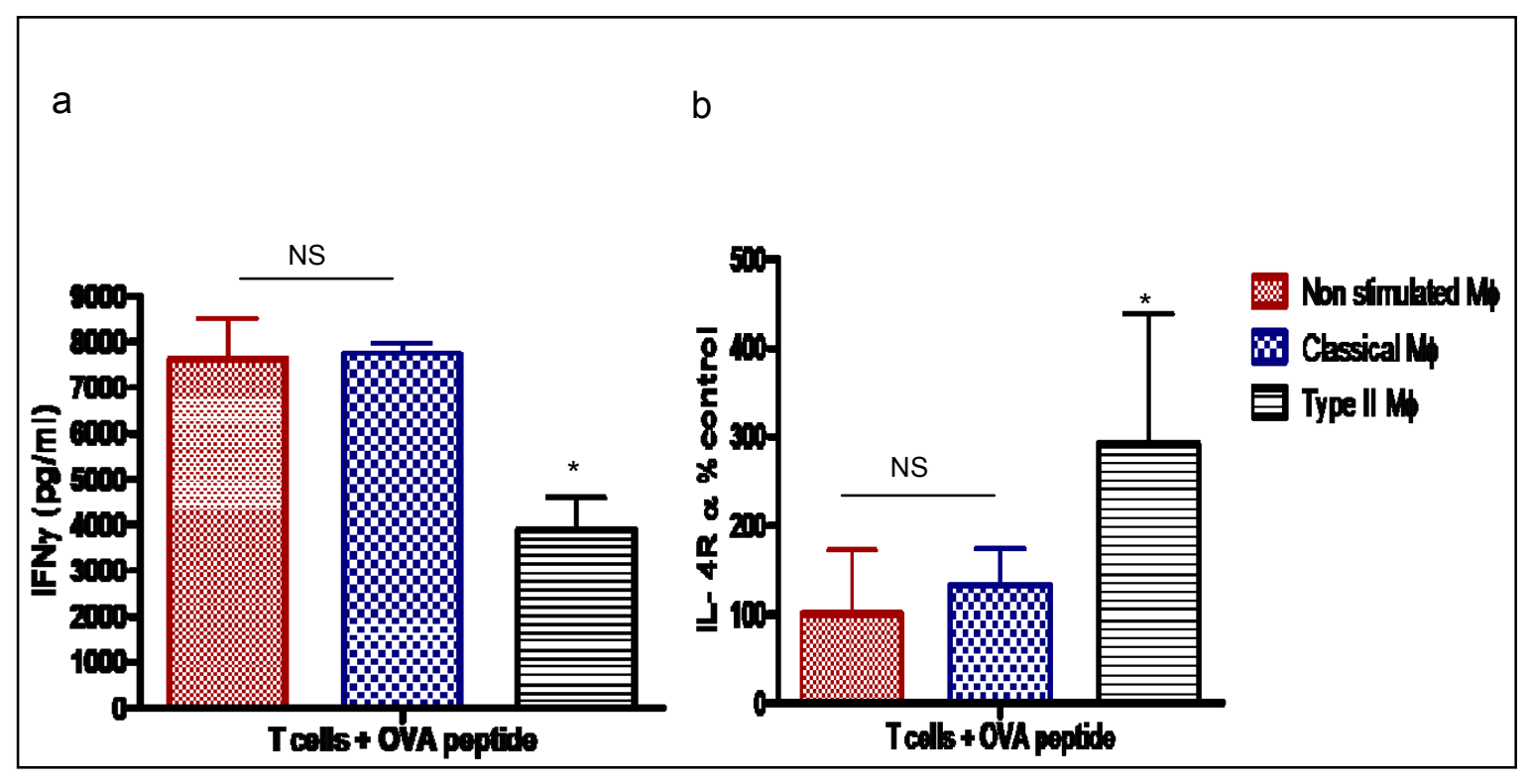

Figure 4.3 Type II activation reduces Th1 while increasing Th2 responsiveness. Macrophages $\left(10^{6}\right.$ cells $/ \mathrm{ml})$ were co-cultured with CD4 T cells $\left(2.5 \times 10^{6}\right.$ cells $\left./ \mathrm{ml}\right)$ as mentioned in Figure 4.1 . After $72 \mathrm{~h}$ culture, IFN $\gamma$ was assessed via ELISA (a), and IL-4Ra was assessed via flow cytometry (b) and presented as \% of control. * $\mathrm{P}<0.05$ : One way ANOVA with Bonferroni post test; Antigen specific responses to type II versus classical MØ. Bars represent data points (mean+SEM) from two experiments. 


\subsubsection{Role of CD40/CD40L interactions during CD4 T cell biasing}

Studies have demonstrated the dual effects of CD40 on dendritic cells and macrophage cell lines, in perpetuating both pro-inflammatory and anti-inflammatory responses depending on the level of CD40 signaling (Murugaiyan et al., 2006). We previously demonstrated type II activated macrophages have reduced levels of CD40, suggesting that the reduced interaction between CD40/CD40L may have a possible role in Th2 cell biasing. In order to identify mechanisms of enhancing Th2 biasing in response to classical and type II activated macrophages, we investigated the effect of reducing the CD40/CD40L interaction between CD4 T cells and macrophages.

IFN $\gamma$ production by $T$ cells exposed to classical and non-activated macrophages is equal, whereas T cells exposed to type II activated macrophages have a significant reduction in IFN $\gamma$ (Figure 4.4). This finding indicates there are less developing Th1 cells in cultures with type II activated macrophages. Upon addition of $0.1-2 \mu \mathrm{g} / \mathrm{ml}$ inhibitory CD40L antibody (MR1), IFN $\gamma$ production is increased under all conditions of macrophage activation (Figure 4.4). At higher levels of the inhibitory antibody (4-8 $\mu \mathrm{g} / \mathrm{ml}), \mathrm{IFN} \gamma$ production is decreased under all macrophage activation conditions (a), such that levels are lower than those seen in the absence of CD40/CD40L inhibition. Comparing the effect of CD40/CD40L inhibition on the relative reduction in IFN $\gamma$ induced by type II macrophages in comparison to classical macrophages, no significant effect can be seen (b). However, with increasing concentrations of inhibitory antibody, T cells exposed to type II activated macrophages maintain reduced IFN $\gamma$ production compared to exposure to classically activated macrophages. This indicates the ability of type II activated macrophages to reduce the development of Th1 cells independently of interactions between CD40 and CD40L.

The effects of CD40/CD40L inhibition on the expression of Th2-associated receptors were also assessed. As shown previously (Figure 4.3), IL-4Ra expression on CD4 T cells in response to classical macrophages is similar to levels in response to non-stimulated macrophages. Furthermore, the level of IL-4Ra expression in response to classically activated macrophages remains unchanged irrespective of CD40/CD40L inhibition (Figure 4.5). IL-4Ra expression in response to type II macrophages is much higher than other culture conditions, but while $2 \mu \mathrm{g} / \mathrm{ml}$ of inhibitory antibody had no real effect, $4-8 \mu \mathrm{g} / \mathrm{ml}$ of inhibitory antibody reduced the expression of IL-4Ra on T cells. IL-2Ra expression, which is normally high in response to type II activation, was similarly reduced in the presence of 0.5-8 $\mu \mathrm{g} / \mathrm{ml}$ of inhibitory CD40L antibody (Figure 4.5 ). At $8 \mu \mathrm{g} / \mathrm{ml}$ of antibody, IL-2Ra expression on $\mathrm{T}$ cells exposed to type II activated macrophages decreased to levels expressed in response to classical and non-stimulated macrophages. 
IL-10 production was assessed in response to CD40/CD40L inhibition. Anti-CD40L antibody at $0.1 \mu \mathrm{g} / \mathrm{ml}$ had no effect on IL-10 in response to type II or classically activated macrophages (Figure 4.6), although IL-10 production in co-cultures with type II activated macrophages was much higher than co-cultures with classically activated macrophages. At $0.5 \mu \mathrm{g} / \mathrm{ml}$ a slight decrease in IL-10 was seen; however at $2-8 \mu \mathrm{g} / \mathrm{ml}$, the levels of IL-10 in response to type II activated macrophages were similar to the levels seen prior to inhibition. At 2-8 $\mu \mathrm{g} / \mathrm{ml}$, in an almost dose dependant fashion, IL-10 production in response to classically activated macrophages significantly increased compared to IL-10 produced in the absence of CD40/CD40L inhibition. This illustrates a possible suppressive effect of CD40/CD40L signaling on IL-10 production. In contrast, IL-12 production remained unchanged by CD40/CD40L inhibition (Figure 4.6), and co-cultures with type II activated macrophages had far lower levels of IL-12 compared to co-cultures with classically activated macrophages. Taken together, these studies demonstrate a necessary role for CD40/CD40L signaling in the induction of Th2 responses by type II activated macrophages, as previously described in dendritic cells (MacDonald et al., 2002a) and found that type II activated macrophages are able to depress Th1 responses irrespective of the level of CD40/CD40L signaling. Also illustrated in this investigation is the suppressive effect of CD40/CD40L signaling on IL-10 production in response to inflammatory stimuli, presenting a new avenue of investigation. 


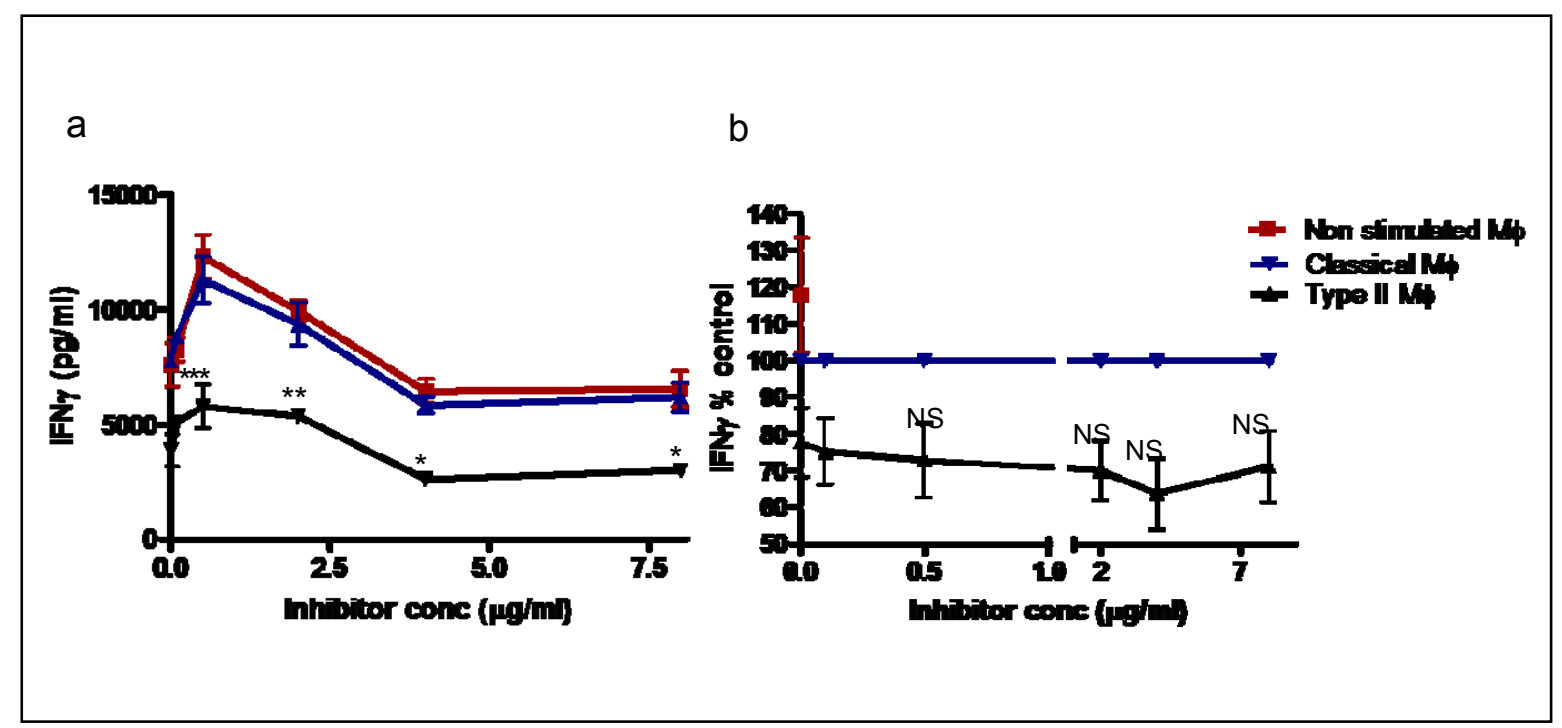

Figure 4.4 Type II macrophages reduce Th1 development independant of CD40/CD40L interactions. Macrophages $\left(10^{6} \mathrm{cells} / \mathrm{ml}\right)$ were exposed to classical or type II inducing stimuli. CD4 T cells were isolated from OTII mice (sec 2.2.4) and stained with CFSE. CD4 T cells $\left(2.5 \times 10^{6}\right.$ cells $/ \mathrm{ml}$ ) plus $1 \mu \mathrm{M} \mathrm{OVA}_{323-339}$ peptide was added to macrophage cultures, along with varying concentrations $(0.1-8 \mu \mathrm{g} / \mathrm{ml})$ of anti-mouse CD40 ligand antibody (MR1) $3 \mathrm{~h}$ after classical or type II stimulation. After $72 \mathrm{~h}$ culture, IFN $\gamma$ was assessed via ELISA. ${ }^{*} P<0.05^{* *} P<0.01{ }^{* * *} P<0.001$ : Two way ANOVA with Bonferroni post test ; Responses to Type II versus Classical MØ. (a) Represents data points (mean + SEM) from duplicate wells of one experiment. (b) Represents combined datapoints (mean + SEM) from two experiments presented as \% of control compared to cultures with classical macrophages. 


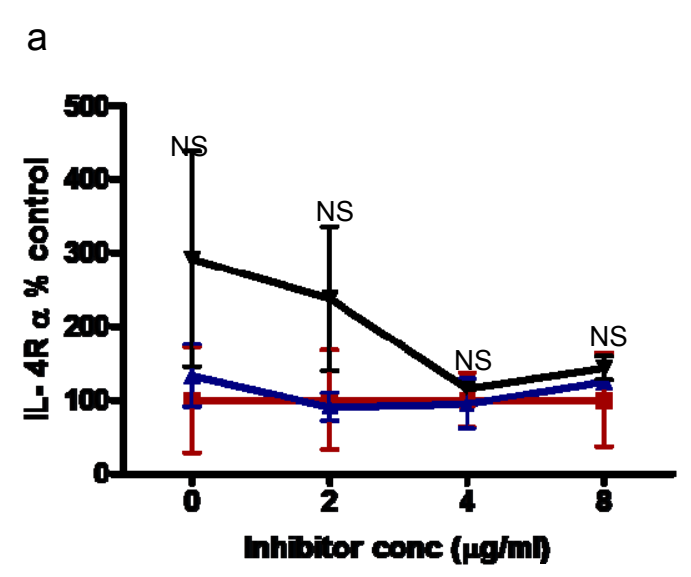

b

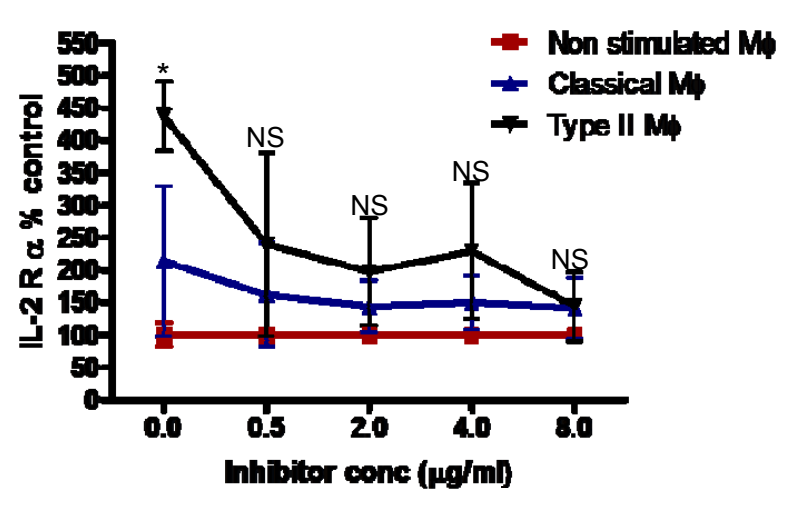

Figure $4.5 \mathrm{CD} 40 / \mathrm{CD} 40 \mathrm{~L}$ inhibition reduces $\mathrm{Th} 2$ responsiveness by type II macrophages. Macrophages were co-cultured with CD4 T cells as mentioned in Figure 4.4. After $72 \mathrm{~h}$ culture IL$4 R \alpha(a)$ and IL-2Ra (b) were assessed via flow cytometry. ${ }^{*} \mathrm{P}<0.05$ : Two way ANOVA with Bonferroni post test; Responses to Type II versus Classical MØ. Graphs represent data points $($ mean + SEM) from two experiments presented as $\%$ of control compared to cultures with classical macrophages.

a

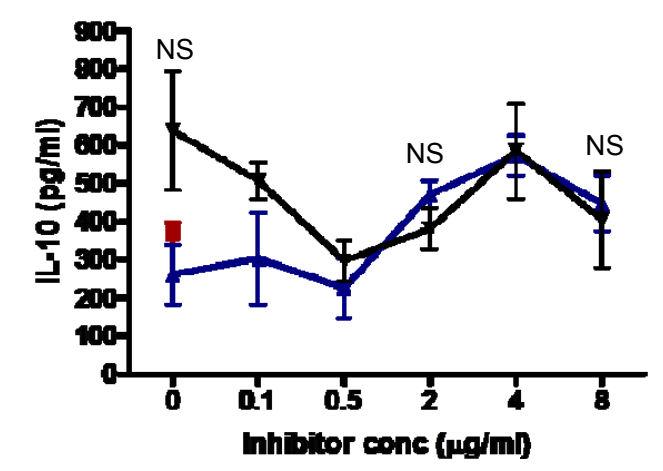

$\mathrm{b}$

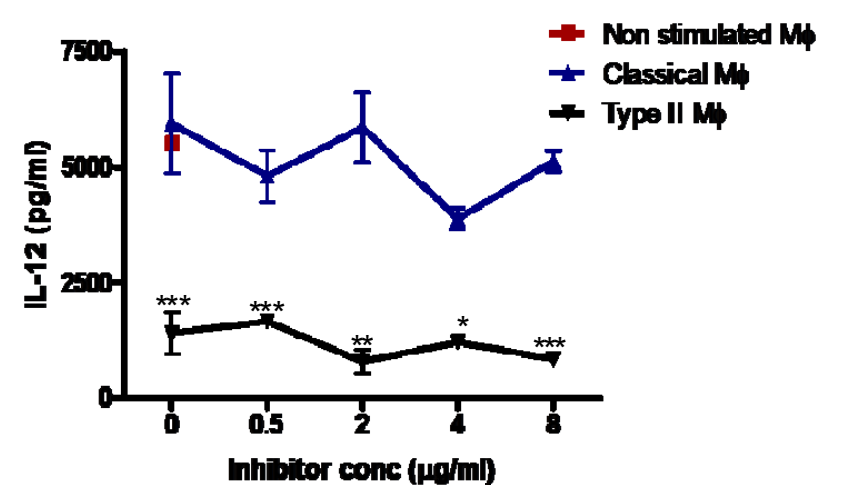

Figure 4.6 CD40/CD40L inhibition increases IL-10 in response to classical macrophages. Macrophages were co-cultured with CD4 T cells as mentioned in Figure 4.4. After $72 \mathrm{~h}$ culture, IL10 (a) and IL-12 (b) were assessed via ELISA. ${ }^{*} \mathrm{P}<0.05{ }^{* *} \mathrm{P}<0.01{ }^{* * *} \mathrm{P}<0.001$ : Two way ANOVA with Bonferroni post test: Responses to Type II versus Classical MØ. Graphs represent data points (mean + SEM) from two experiments, presented as \% of control compared to cultures with classical macrophages.

The red dot in (4.4b) and (4.6a-b) represent the average level of cytokines in co-cultures with non-stimulated macrophages. [see appendix A for complete graphs] 


\subsection{Discussion}

Earlier investigations found that signalling through CD40 and IL-10 did not play an important role in defining the phenotypic characteristics of type II macrophages (Chapter 3). However, these experiments did not assess the effects of the CD40/CD40L interaction between macrophages and CD4 T cells in co-cultures. CD40L signalling on CD4 T cells is essential for promoting an appropriate response to antigen (Grewal et al., 1998). In recent years, it has come to light that $\mathrm{CD} 40 \mathrm{~L}$ is not only essential for the induction of inflammatory Th1 responses (Alderson et al., 1993; Florido et al., 2004), but also in the development of Th2 responses (MacDonald et al., 2002a; Poudrier et al., 1998), whereby a deficiency in CD40L results in an inability to elicit protective Th2 responses during infection with a parasitic helminth. These different observations on the effect of CD4OL inhibition on $\mathrm{T}$ cell responses may correspond to the dual ability of macrophages to perpetuate pro-inflammatory and anti-inflammatory responses (Mathur et al., 2004). Type II activated macrophages protect against EAE with Th2 biasing implicated in the protective effects (Tierney et al., 2009). Because CD40 levels are significantly reduced on type II activated macrophages, it is possible that type II activated macrophages have low level CD40/CD40L interactions with T cells, which influences T cell biasing (La Flamme et al., 2006; Tierney et al., 2009). Therefore, identifying the role of the reductions in CD40 on T cell biasing may enhance our understanding of how type II activated macrophages protect against pro-inflammatory diseases.

In order to further investigate mechanisms involved in T cell biasing by type II activation, studies were carried out to elucidate the effects of type II activated macrophages on naive $\mathrm{T}$ cell function (e.g. proliferation, cytokine production) upon first antigenic exposure. A previous study demonstrated that type II activated macrophages had a significantly reduced expression of PDL-1 (Tierney et al., 2009). This co-signalling molecule has been associated with T cell suppression through its interaction with PD-1 (Keir et al., 2006), but has also been implicated in T cell stimulation under certain conditions (Tseng et al., 2001), thus making it difficult to predict the role of PDL-1 reductions by type II activation. As type II activated macrophages exhibit a significant reduction in this molecule in comparison to classical activation, it was suggested by Edwards et al (2006) and Tierney et al (2009) that type II activation may promote greater $\mathrm{T}$ cell proliferation than classical activation. However, we found that CD4 T cells exposed to classical and type II activated macrophages proliferated similarly in response to OVA peptide or protein (antigens). This finding conflicts with those of Edwards et al where a slight increase in proliferation in response to the type II activated macrophages was reported (Edwards et al., 2006).

One possible explanation for the difference may be in the experimental systems used. Edwards et al used IgG-coated OVA as the immune complex and used T cells from the 
DO11.10 TCR transgenic mouse line while our system utilized IgG-coated SRBC as the immune complex and T cells from OTII mice. Although both recognise the same core amino acid within OVA protein, it is possible that differences in other TCR contact residues between the two cells resulted in different responses (Robertson et al., 2000). Additionally, Edwards et al reported enhanced T cell proliferation to type II activation, however as PDL-1 was not the focus of their investigation, levels were not assessed. Thus, no direct correlation between PDL-1 and proliferation was discussed.

This investigation indicates that the significant reduction observed in PDL-1 expression by type II activation does not lead to enhanced CD4 T cell proliferation over that observed with classically activated macrophages. This finding could suggest a role of PDL-1 other than its inhibitory functions on $T$ cells. Such a role may include $T$ cell activation and/or $T$ cell biasing. In support of this idea, previous literature demonstrate effects of PDL-1 that are unrelated in their anti-proliferative role (Loke et al., 2003; Tseng et al., 2001; Zhu et al., 2006). Using different murine models of EAE, Zhu et al (2006) demonstrated instances in which PDL-1 blockage had no effect on disease progression, irrespective of the reported role of PDL-1 in limiting auto-reactive responses (Keir et al., 2006). Furthermore, Loke et al (2003) illustrated the differential regulation of PDL-1 and PDL-2 by counterregulatory Th1/Th2 responses respectively, implicating a possible role for the different ligands in the counter-regulatory $\mathrm{T}$ cell responses. Therefore, the observed reductions in PDL-1 on type II activated macrophages may serve a role in Th1/Th2 cell biasing as opposed to T cell proliferation.

Prior to carrying out further investigations into the mechanisms involved in T cell biasing, another goal was to validate the induction of $\mathrm{T}$ cell biasing by type II activation using our system. This investigation also set out to identify ways of assessing $T$ cell biasing for future investigations using the same system.

IFN $\gamma$ is a prototypic cytokine produced by Th1 cells (Abbas et al., 1996; Szabo et al., 2000), involved in the induction of Th1 responses which aid the activation of antimicrobial, pro-inflammatory responses by macrophages and surrounding $T$ cells (Alderton et al., 2001; Campbell et al., 1996; Li et al., 1997; MacMicking et al., 1997). The prototypic cytokine for developing Th2 cells is IL-4 (Abbas et al., 1996; Kaplan et al., 1996; Le Gros et al., 1990; Seder et al., 1992). Our studies showed that T cells exposed to either non activated or classically activated macrophages produce high amounts of IFN $\gamma$, indicative of Th1 cell biasing. In contrast, T cells exposed to type II activated macrophages produced significantly less IFN $\gamma$ compared $\mathrm{T}$ cells cultured with classically activated macrophages. This reduction in IFN $\gamma$-producing cells is indicative of a reduction in Th1 
development in response to type II macrophage activation, corroborating with previous literature (Gerber et al., 2001; La Flamme et al., 2006).

Although IL-4 is the best marker for Th2 polarization, only very low levels of IL-4 were detected in the $T$ cell cultures. These low levels of IL-4 may be due to the long time IL-4 takes to be produced by T cells (Bird et al., 1998), along with re-uptake of IL-4 through the IL-4 receptor on T cells. As we were unable to assess Th2 development by IL-4 detection, the effects of type II activated macrophages on the expression of surface markers associated with developing Th2 cells was assessed. IL-4Ra as well as IL-2Ra are implicated in increasing the responsiveness of CD4 T cells to Th2-inducing cytokines. Signalling through IL-2Ra via the STAT-5 signalling molecule is essential in the expression of IL-4Ra (Liao et al., 2008), as well as the production of IL-4 in CD4 T cells (Cote-Sierra et al., 2004). In this investigation, very little IL-4Ra was expressed on cells exposed to non-activated or classically activated macrophages. In contrast, IL-4Ra expression was up-regulated on T cells exposed to type II activated macrophages, and this increase in IL-4Ra corresponded to an increase in IL-2Ra expression, further supporting the relationship between IL-4R $\alpha$ and IL-2R $\alpha$ expression. Although type II activation has previously demonstrated the increase in IL-2Ra (Edwards et al., 2006), this is the first demonstration of such a large increase in IL-4Ra by type II activation. Furthermore, previous studies have not assessed the involvement of IL-2Ra expression in T cell biasing as opposed to being used as an indicator of general activation by type II activated macrophages. Although much remains to be investigated to fully elucidate a direct correlation in the setting of type II activation, previous reports have demonstrated a direct link between IL-2Ra, IL-4Ra and increased IL-4 responsiveness (Cote-Sierra et al., 2004; Liao et al., 2008). This suggests type II activation increases the responsiveness of CD4 T cells to Th2-inducing cytokines, while reducing the development of IFN $\gamma$-producing Th1 cells. This Th2 cell biasing corresponds with previous observations (Anderson et al., 2002; Tierney et al., 2009), and validates the use of IL-4Ra as well as IL-2Ra in conjunction with cytokine profiles, to identify Th2 cells.

Although data presented on IL-2Ra expression is preliminary, it warrants further investigation in light of recent literature supporting a role of IL-2 in many regulatory responses not associated with proliferation. This project has not yet investigated the effects of type II activated macrophages on regulatory $T$ cell induction which is also associated with increased IL-2 responsiveness (Pandiyan et al., 2007); however, the lack of inhibition on $\mathrm{T}$ cell proliferation supports the conclusion that Tregs are not the dominant subset induced. Further studies looking at FoxP3, a widely used marker of Tregs, is required to ascertain the exact involvement of inducible Tregs (iTregs) in protection mediated by Type II activated macrophages (Roncarolo et al., 2008), as it is possible that 
the cytokine environment and phenotype induced by type II activation supports the development of Th2 as well as iTregs, both of which are associated with protection against EAE.

Th1 cells are involved in promoting pathological processes during EAE (Becher et al., 2002), with administration of the Th1 cytokine IFN $\gamma$ resulting in exacerbations of MS , and levels of IFN $\gamma$ detected at the peak of disease (Begolka et al., 1998). Therefore the reduction in IFN $\gamma$ demonstrated by type II activated macrophages is a contributing factor to the prevention of Th-1 mediated disease. Th2 responses are associated with protection from inflammatory diseases such as EAE (Cua et al., 1995; Shaw et al., 1997; Weber et al., 2007), and the protection type II activation provides against EAE is associated with Th2 polarisation (Tierney et al., 2009). Treatments which enhance Th2 development, such as glatiramer acetate (GA), are also beneficial in the treatment of MS (Weber et al., 2007). Therefore, as well as the ability of type II activated macrophages to reduce inflammatory responses by macrophages, the reduction in Th1 T cell responses and increase in Th2 T cell responses elicited by type II activation presents beneficial effects against inflammation. Given that these findings demonstrate the ability of type II activated macrophages to alter $\mathrm{T}$ cell biasing, the next step is to identify mechanisms involved in promoting this effect.

This thesis focused on the role of CD40, as a role for CD40 in promoting both Th1 and Th2 responses has been reported (Becher et al., 2000; MacDonald et al., 2002a; MacDonald et al., 2002c). Because CD40 expression is significantly reduced on type II activated macrophages, we set forth to investigate the effect of altered levels of CD40/CD40L signalling on $\mathrm{T}$ cell biasing. The different levels of CD40/CD40L signalling were regulated by titrating the concentration of anti-CD40L blocking antibody. Blockage between CD40 and CD40L resulted in reduced general responses of $\mathrm{T}$ cells as exhibited by the reduced levels of IFN $\gamma$ detected in all co-cultures. This reduction agrees with previous literature emphasizing the requirement for CD40 signaling to elicit IFN $\gamma$ production by Th1 cells (Alderson et al., 1993; Campbell et al., 1996). However, contradictory to this is the lack of effect of full CD40/CD40L inhibition on the production of IL-12. Additionally, co-cultures with type II activated macrophages maintained significantly lower levels of IL-12 compared to classically activated macrophages, and their general production of IL-12 in response to either type II or classical activation remained unaltered irrespective of the level of CD40 inhibition. This finding corresponds to the previous observation where alterations in CD40 signaling did not affect the cytokine profiles of type II activated macrophages, but contradicts studies which found that CD40/CD40L signaling was essential for antigen-specific IL-12 production derived from activated macrophages, dendritic cells and microglia (Becher et al., 2000; DeKruyff et al., 1997; Kelsall et al., 
1996). One possible reason for the lack of alteration in IL-12 production by CD40/CD40L inhibition, is that IL-12 production in the presence of a strong Th1-inducing stimulus such as LPS, occurs independent of CD40 ligation (Reis e Sousa et al., 1997). As LPS is involved in the induction of both type II and classical activation, this explanation has merit.

The ability of type II activated macrophages to reduce IFN $\gamma$-producing Th1 cells is maintained irrespective of full inhibition of CD40/CD40L interactions, indicating this reduction occurs independently of the CD40/CD40L interaction, as does the reduction in IL-12 production. This result illustrates a possible relationship whereby the ability of type II activated macrophages to maintain reduced IL-12 production is associated with the maintained reduction of Th1 development. In addition to Th17 cells, Th1 cells constitute an important cell type involved in the pathogenesis of EAE (Becher et al., 2002; Langrish et al., 2005). Since IL-12 is important in the development of IFN $\gamma$-producing Th1 cells (Howard et al., 1999; Kelsall et al., 1996; Seder et al., 1996), the maintained reduction of this cytokine by type II activation may be key to inhibiting Th1 development and promoting protective effects against EAE.

Even though the reduction in Th1 development mediated by type II activation occurs independently of CD40/CD40L, we wished to identify whether this interaction was essential for Th2 biasing by type II activated macrophages, as seen previously by dendritic cells (MacDonald et al., 2002c). With $2 \mu \mathrm{g} / \mathrm{ml}$ of inhibitory CD40L antibody, there is a slight but non-significant reduction in the expression of IL-4Ra on T cells exposed to type II activated macrophages. Under these conditions, these cells still exhibit much higher levels of expression compared to $T$ cells exposed to non-activated or classically activated macrophages indicating low level inhibition of CD40/CD40L still enables Th2 biasing by type II activation while maintaining the inhibitory effect on Th1 development. However $4-8 \mu \mathrm{g} / \mathrm{ml}$ of inhibitory antibody reduces the expression of IL-4Ra expression in response to type II activation, to levels detected on $\mathrm{T}$ cells exposed to non-activated macrophages. A similar effect can be seen with the expression of IL-2Ra, which dramatically decreases upon CD40/CD40L inhibition. At full inhibition of the CD40/CD40L interaction $(8 \mu \mathrm{g} / \mathrm{ml})$, a complete reduction in the expression of IL-2Ra can be seen, illustrating the dependency of Th2 responsiveness on CD40/CD40L signaling. The lack of effect low levels of CD40/CD40L inhibition have on IL-4 responsiveness suggests a possible explanation for the induction of Th2 polarization upon administration of antiCD40L antibody reported by Hancock et al (2006). Although this is not the first report of a requirement for CD40L signaling in Th2 development (MacDonald et al., 2002a), it is the first study to demonstrate the requirement for this interaction in Th2 biasing by type II activation. 
Our previous studies indicated IL-10 derived from macrophages did not have a significant effect on the macrophage phenotype elicited by type II activation. However, IL-10 derived from macrophages and T cells can be different both in their regulation (Shoemaker et al., 2006), and their function (Roers et al., 2004). Therefore, we assessed the effect of CD40/CD40L alterations on IL-10 production by $\mathrm{T}$ cells exposed to type II activated macrophages. IL-10 production in co-cultures with type II activated macrophages remained higher than levels detected in co-cultures with classically activated macrophages and were not further enhanced by CD40/CD40L inhibition. However, cocultures with classically activated macrophages exhibited increased production of IL-10, in a dose dependant manner with increasing inhibition of CD40/CD40L. At full CD40/CD40L inhibition, the level of IL-10 in co-cultures with classically activated macrophages equaled the levels of IL-10 in co-cultures with type II activated macrophages. This indicates CD40/CD40L interactions may be having a suppressive effect on IL-10 production, which has not been previously reported.

In summary, these studies uncovered a suppressive effect of CD40/CD40L on IL-10 production, which could be overcome by inhibiting the CD40/CD40L interaction. The resulting alterations in IL-10 may be a contributing factor to the regulation of Th1 and Th2 responses seen at high and moderate levels of CD40/CD40L signaling respectively. These findings also provide a greater understanding into how CD40 inhibition prevents Th2 development, as previous studies have not investigated the effects of CD40 inhibition on the receptors for IL-4 and IL-2, or on the production of the suppressive cytokine IL-10. Although we were unable to enhance Th2 biasing by type II activated macrophages, we now have a greater understanding of some of the critical mechanisms involved in Th2 and Th1 biasing. 


\section{Chapter 5: Effects of schistosome complexes on macrophage activation}

\subsection{Introduction}

In recent times, a strong negative correlation between the exposure to infectious diseases and the development of autoimmunity has been reported (Cook, 1996; Fleming et al., 2007), suggesting that chronic infection with some parasites provides a level of protection against autoimmune diseases. For example, schistosomiasis has been shown to provide protection from autoimmune diseases such as type1 diabetes, graves thyroiditis and multiple sclerosis, in mouse models of these diseases. Interestingly, exposure to the schistosome egg (Cooke et al., 1999; Sewell et al., 2003) or soluble egg extract (SEA) (Zaccone et al., 2003; Zheng et al., 2008), and not just live infection (La Flamme et al., 2003), provides similar protection. This suggests that it is the immune response to the eggs which regulate autoimmune responses. While it has been shown that this protection is dependent on a polarised Th2 response (Sewell et al., 2003), the mechanism by which schistosome infection provides protection is not known.

Previous research (Herbert et al., 2004) found that alternatively-activated macrophages were important during schistosomiasis as their absence resulted in severe morbidity despite the presence of a strong Th2 response. This study indicates that during schistosomiasis, macrophages regulate immune responses and promote survival. To date, the main role for alternatively activated macrophages has been associated with their role in wound repair/fibrogenesis (Albina et al., 1990) and the down-regulation of inflammatory responses (Herbert et al., 2008; Pesce et al., 2009a). These macrophages can be identified by various markers, some of which include up-regulation of arginase 1 and RELMa (Loke et al., 2003; Modolell et al., 1995; Raes et al., 2002). Thus, one possible mechanism by which schistosome infection may protect from autoimmunity is by altering macrophage activation.

Macrophages exhibit extreme plasticity (Mosser et al., 2008; Stout et al., 2004), and can alter their phenotypic properties accordingly with the situation at hand. While it has been shown that schistosome infection leads to alternative activation of macrophages, it is also possible that other states of activation occur as well, such as type II activation. Furthermore, if these different activation states do occur during schistosomiasis, it is reasonable to presume that these states may also be contributing to protection from proinflammatory diseases such as EAE. In support of this idea, recent studies have shown that altering macrophage activation to support type II macrophages protects from EAE and the protection is Th2-dependant (La Flamme et al., 2006; Tierney et al., 2009). Thus, 
we wished to examine whether schistosome immune complexes can induce type II macrophage activation in vitro.

Aims:

The mechanism by which schistosome infection protects from EAE and other proinflammatory diseases is still under study, although it is clear that it is Th2-dependant (La Flamme et al., 2003; Sewell et al., 2003; Zheng et al., 2008). Type II macrophages induce Th2-biased responses (Anderson et al., 2002), but no study to date has identified whether macrophages are activated into a type II state during schistosome infection. Type II activation is typically induced via Fc $\gamma$ receptor ligation by exposure to immune complexes in the presence of inflammatory stimuli. During schistosome infection, high levels of schistosome-specific antibodies and immune complexes are in the blood and tissue (Dunne et al., 2005; Santoro et al., 1979), as well as inflammatory stimuli caused by leakage of bacterial components due to egg passage through the intestine (Herbert et al., 2008). Therefore, the environment generated during schistosome infection is capable of inducing type II macrophage activation. Indeed, it has been demonstrated that macrophages exposed to LPS in the presence of SEA or SEA complexed with serum from an infected mouse, produce far less IL-12 and increased IL-10 (La Flamme et al., 2004), suggesting that type II activation does occur. We aim to investigate fully the effects of schistosome immune complexes on macrophages in vitro to determine whether type II activation occurs. To this end, we addressed 2 specific aims:

1. To identify the cytokine and surface marker profiles on murine bone barrow derived macrophages induced by schistosome whole egg complexes (Egg:Serum), and soluble egg antigen immune complexes (SEA :lgG), in order to elucidate responses indicative of type II activation.

2. To determine if schistosome egg antigen complexes (SEA:lgG) induce a similar enzymatic profile to that of type II macrophages or to that of other known activation states (e.g. alternative or classical). 


\subsection{Results}

\subsubsection{Anti-inflammatory effect of schistosome egg}

To investigate the effects of schistosome eggs on macrophage activation, primary bone marrow-derived macrophages were stimulated with LPS (i.e. classically activated) in the presence of either schistosome egg, or egg opsonised with serum from a schistosome infected mouse (i.e. immune complex). Because schistosome eggs are very large and polyvalent, it was expected that they would be good at ligating $\mathrm{Fc} \gamma$ receptors on macrophages and inducing type II activation. Cytokine analysis of culture supernatants showed that while LPS-stimulated macrophages produced high levels of IL-12 and low levels of IL-10, stimulation of macrophages with LPS in the presence of either egg or egg complex resulted in reduced levels of IL-12 and increased IL-10 (Figure 5.1), similar to the effect seen with type II activation by opsonised SRBC (Chapter3). In the absence of LPS, stimulation with either egg or egg complexed with infected serum (Egg:Ser) elicited the same response as non-stimulated macrophages. Kinetic studies indicate the most significant reduction in IL-12 and increase in IL-10 occur $8 \mathrm{~h}$ post stimulation as was noticed previously with type II activated macrophages. Interestingly, the modulating effects of schistosome eggs or eggs complexed with infected serum on the responses to LPS were very similar (Figure 5.1), suggesting that the alterations in macrophage responses may be occurring via a non-Fc $\gamma R$ pathway.

Unlike stimulation with opsonised SRBC, stimulation with LPS plus schistosome eggs or eggs complexed with infected serum only moderately alters the expression of costimulatory molecules (Figure 5.2). While LPS-induced CD80 expression was slightly reduced by egg or egg complex $24 \mathrm{~h}$ post-stimulation, CD40 expression was unaffected except at $24 \mathrm{~h}$ where a reduction can be seen by both egg and egg complex in the presence of LPS. PDL-1, like CD40, remained unaffected 2-8 h post stimulation but reduced slightly $24 \mathrm{~h}$ after stimulation with schistosome egg plus LPS. This effect was not seen with schistosome egg complex. Overall, there is a small reduction in the expression of activation/co-stimulatory markers $24 \mathrm{~h}$ after treatment with schistosome egg or egg complex compared to classical activation. However, these reductions in expression are significantly less than those seen by type II activated macrophages. Moreover, because the $24 \mathrm{~h}$ time point was only examined once, the consistency of this reduction needs verification.

Taken together, these data indicate that schistosome whole eggs either alone or as an immune complex, are capable of activating macrophages into a state which exhibits an anti-inflammatory cytokine profile. Although the kinetics of the cytokine alterations are similar to type II activation, the expression profile of co-stimulatory markers differs to that 
of type II activated macrophages. These findings, in addition to the similarity in responses to the egg immune complex and eggs alone in the presence of LPS, suggest that type II macrophage activation may not be induced by schistosome egg complexes. Rather, they indicate that the anti-inflammatory profile is generated through another unknown mechanism that warrants further investigation. 


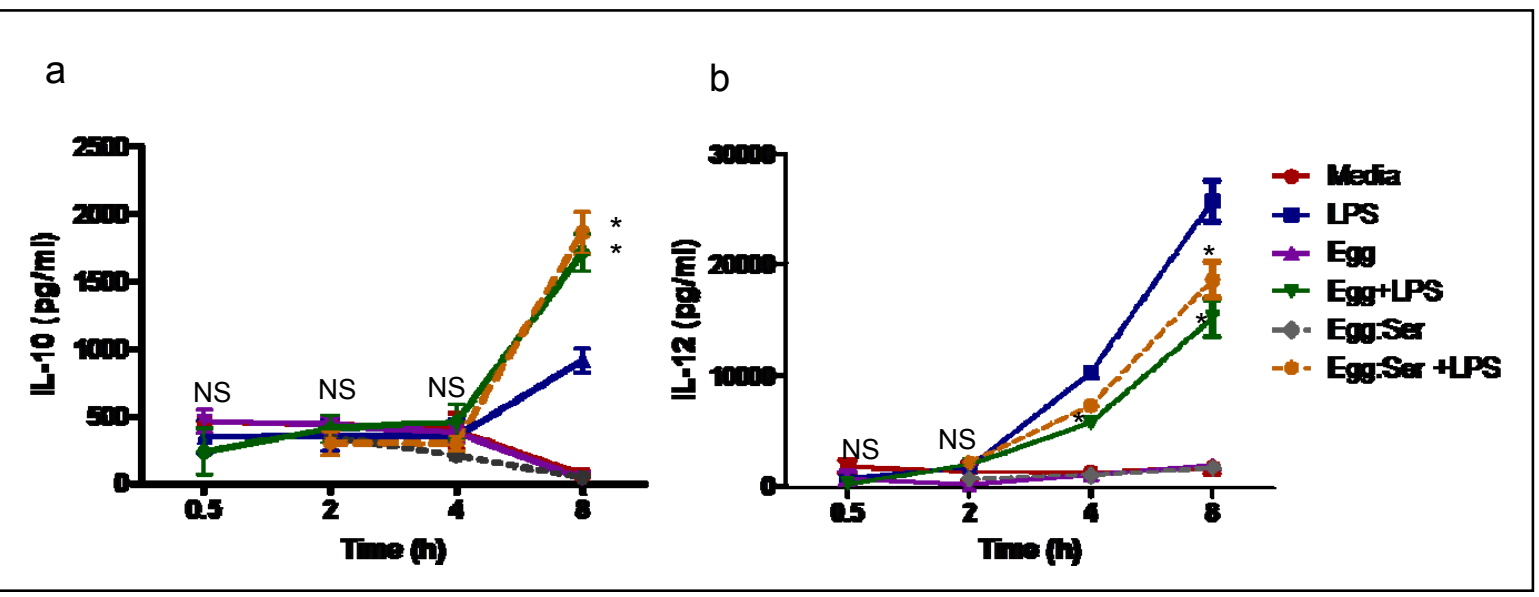

Figure 5.1 Schistosome egg and egg complex reduce LPS-induced IL-12 and increase IL-10. Primary BMM $\varnothing\left(10^{6} \mathrm{cells} / \mathrm{ml}\right)$ derived from C57BL/6 mice were primed with $20 \mathrm{U} / \mathrm{ml} \mathrm{IFN} \gamma$ overnight followed by stimulation with Media, LPS (200 ng/ml), Schistosome egg (1000/well), Egg + LPS, opsonised Egg (Egg:Serum from schistosome infected mouse), or opsonised Egg + LPS. After 0.5, 2, 4 and $8 \mathrm{~h}$ culture IL-10 (a) and IL-12 (b) were assessed via ELISA. ${ }^{*} \mathrm{P}<0.001$ : Two way ANOVA followed by Bonferroni post test; Egg + LPS or Egg:Serum + LPS versus LPS. Graphs represent the mean + SEM from combined duplicate wells of two experiments. 


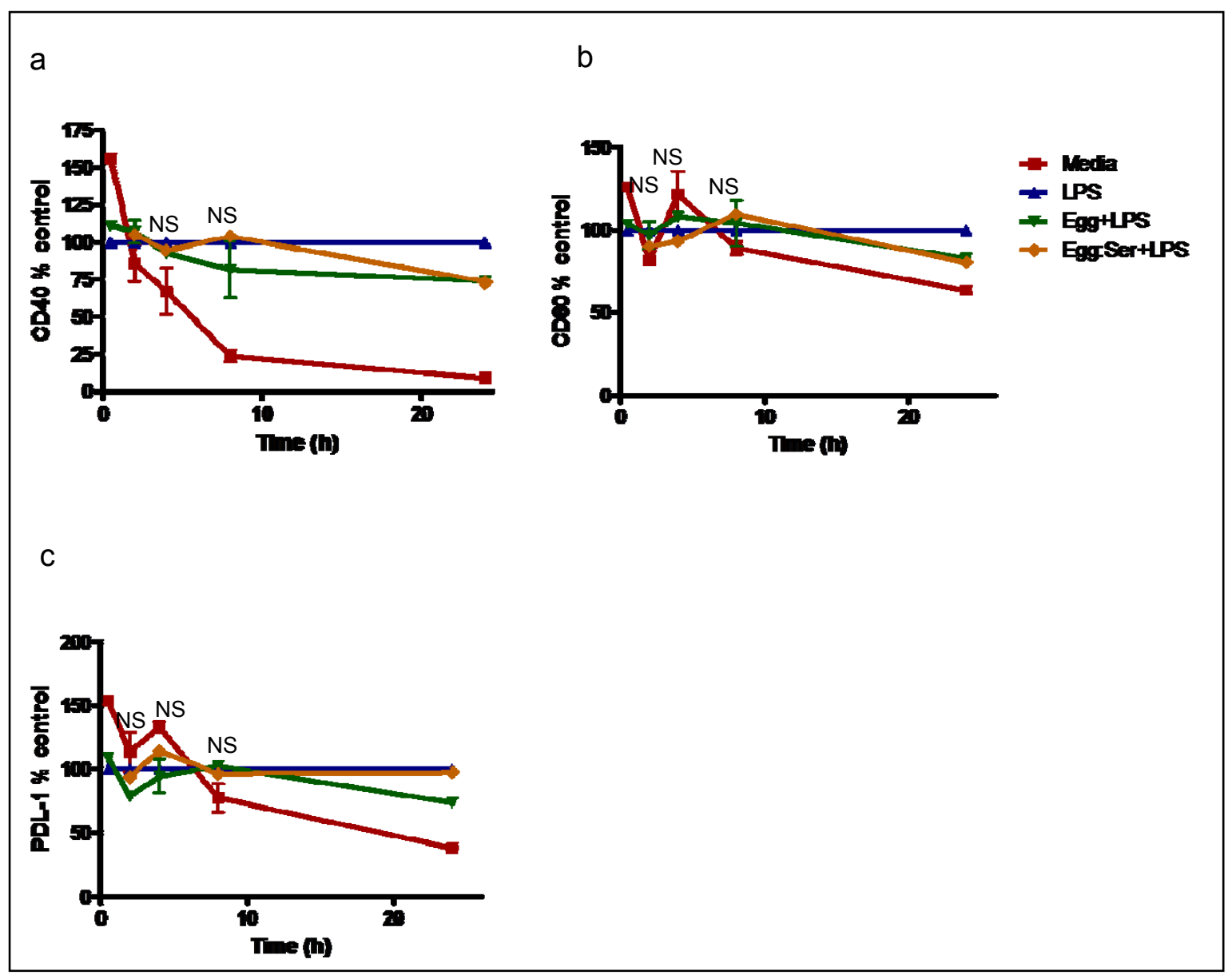

Figure 5.2 Schistosome egg and egg complex induce no significant alterations in macrophage surface markers. Primary BMM $\varnothing\left(10^{6}\right.$ cells $\left./ \mathrm{ml}\right)$ derived from C57BL/6 mice were treated as described in Figure 5.1. After 0.5, 2, 4, 8 and 24 h culture CD40 (a), CD80 (b) and PDL-1 (c) were assessed via flow cytometry. Difference between Egg + LPS or Egg:Ser + LPS versus LPS is NS $P>0.05$ : Two way ANOVA with Bonferroni post test. Data represents the mean + SEM of two experiments. The $24 \mathrm{~h}$ time point represents one experiment. Data is presented as $\%$ of control compared to cultures with LPS. See Appendix A for full graphs with stimulants in the absence of LPS. 


\subsubsection{Ability of SEA/SEA complexes to activate macrophages of an anti- inflammatory nature}

Because immune complexes consisting of soluble schistosome antigen (SEA) and not necessarily whole eggs circulate in the blood of infected subjects, the effect of schistosome soluble egg antigen immune complexes on macrophage activation was assessed. Primary murine bone marrow-derived macrophages were stimulated for $8 \mathrm{~h}$ and $24 \mathrm{~h}$ with LPS in the presence or absence of SEA or SEA complexed with purified IgG (SEA-IgG) from schistosome-infected mice, and compared to type II activation induced by opsonised SRBC. To determine the optimum ratio of SEA to purified IgG, various ratios of SEA and IgG were used and the reduction in LPS-stimulated IL-12 production was assessed. Although SEA alone induced a reduction in IL-12, the addition of purified IgG enhanced this reduction. Figure 5.3 indicates the optimal ratio of SEA to $\lg$ is $5 \mu \mathrm{g} / \mathrm{ml}$ SEA to $20 \mu \mathrm{g} / \mathrm{ml}$ of purified lgG, as SEA-lgG ratios of $5-10 \mu \mathrm{g}, 10-10 \mu \mathrm{g} / \mathrm{ml}$ or $10-20 \mu \mathrm{g} / \mathrm{ml}$ induce a less significant reduction in IL-12 compared to the $5-20 \mu \mathrm{g} / \mathrm{ml}$ SEA-lgG ratio.

Using this optimal ratio of SEA-IgG, IL-10 production was assessed by macrophages treated with SEA complexes. Figure 5.4 indicates at the optimised ratio of SEA complex, the reduction in IL-12 was greater than that seen with type II activation and the increase in IL-10 was equal to the increase by type II activation. As type II activated macrophages are known to induce Th2 biasing, IL-4 expression was also assessed under the same conditions. As expected, classically activated macrophages (LPS) produced nearly undetectable levels of IL-4. However, while type II activated macrophages (SRBC complex plus LPS) produced low but detectable levels of IL-4, stimulation of macrophages with SEA complexes in the presence of LPS induced a significant increase in IL-4 production. This is a novel finding, and the potential role of macrophage-derived IL-4 during schistosome infection warrants further investigation.

In order to investigate the effects of SEA complexes on co-stimulatory marker expression, expression levels were compared to that seen with type II activated macrophages and alternatively activated (i.e. IL-4-stimulated) macrophages. Figure 5.5 shows LPS stimulation induces a strong up-regulation in CD40 and a modest increase in CD80 and PDL-2 expression compared to non-stimulated macrophages. In contrast, macrophages exposed to LPS in the presence of SEA-complexes showed reduced levels of CD40 and CD80 expression compared to classical activation, and reduced the expression of PDL-2, which was up-regulated upon exposure to rlL-4. Based in this data, SEA-complexes induce a co-stimulatory marker and cytokine profile similar to that of type II activated macrophages, suggesting that SEA complexes may be inducing type II macrophage activation. Furthermore, there was a significant increase in IL-4 in response to SEA- 
complexes, which was not found in the other activation conditions (classical, type II or alternative). Therefore further investigations are merited to investigate whether type II activation occurs or whether a novel activation state has been induced. 


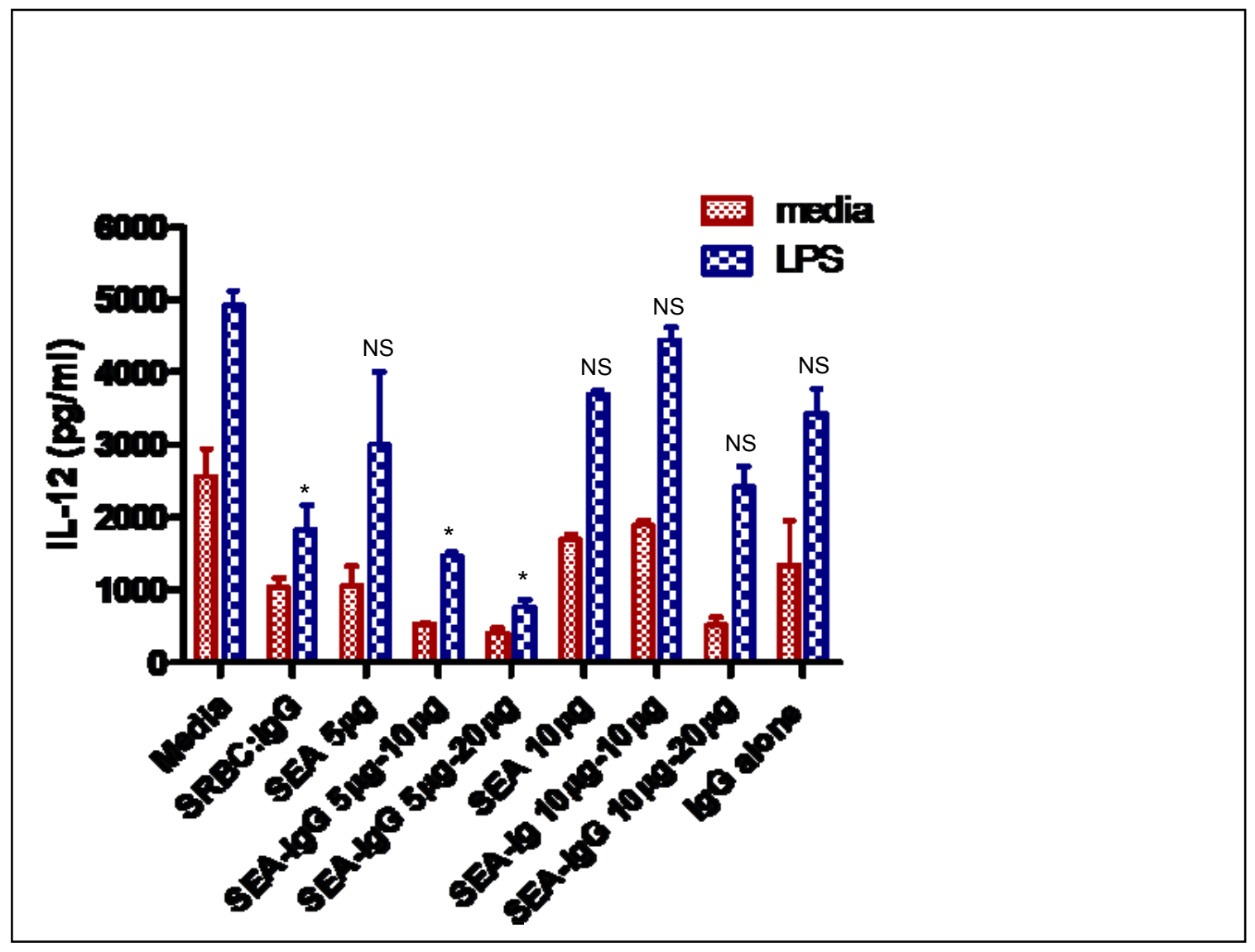

Figure 5.3 Schistosome complex of $5-20 \mu \mathrm{g} / \mathrm{ml}$ elicits the greatest reduction in LPS-induced IL-12. As mentioned in Figure 5.2, primary BMM $\varnothing$ were primed overnight. Macrophages were then stimulated both in the presence and absence of LPS $(200 \mathrm{ng} / \mathrm{ml})$, with $5 \mu \mathrm{g} / \mathrm{ml}$ or $10 \mu \mathrm{g} / \mathrm{ml} \mathrm{SEA}$, or SEA-IgG complex at various ratios of SEA bound to purified IgG $(5 \mu \mathrm{g} / \mathrm{ml}$ or $10 \mu \mathrm{g} / \mathrm{ml} \mathrm{SEA}$, with 10 $\mu \mathrm{g} / \mathrm{ml}$ or $20 \mu \mathrm{g} / \mathrm{ml} \mathrm{lgG}$ ). Cells were also classically (LPS) or type II activated (SRBC:IgG+LPS). After $8 \mathrm{~h}$ culture, IL-12 was assessed via ELISA. ${ }^{*} \mathrm{P}<0.05$ : One way ANOVA; Conditions versus LPS. Data represents the mean+SEM from duplicate wells of one experiment. 


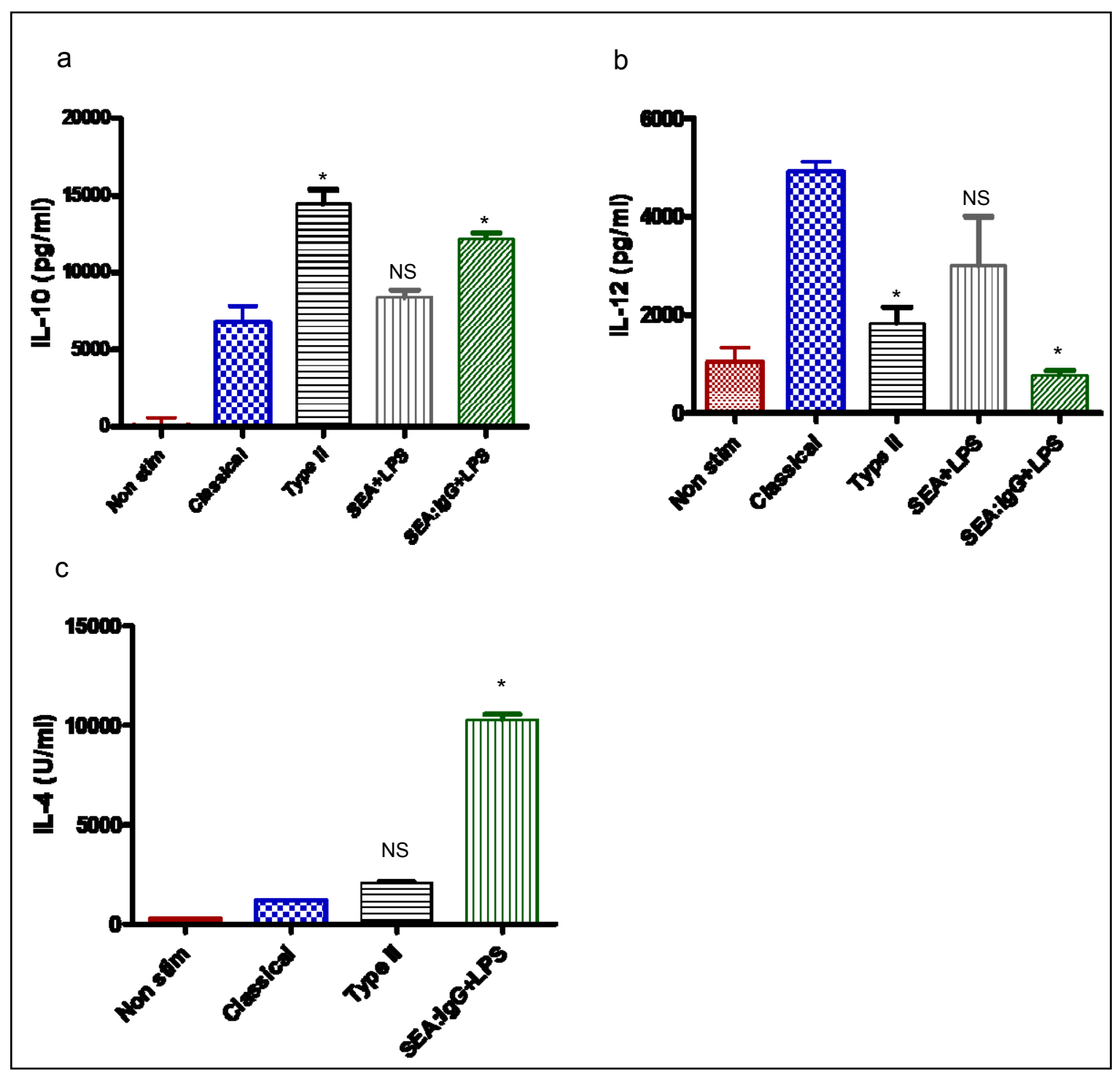

Figure 5.4 SEA complexes elicit a similar IL-10/IL-12 ratio to type II macrophages but differ significantly in IL-4 induction. Primary BMM $\varnothing\left(10^{6}\right.$ cells $\left./ \mathrm{ml}\right)$ derived from C57BL/6 mice were primed with $20 \mathrm{U} / \mathrm{ml} \mathrm{IFN} \gamma$ overnight followed by exposure to $5 \mu \mathrm{g} / \mathrm{ml}$ SEA or SEA-lgG $(5 \mu \mathrm{g} / \mathrm{ml}-20$ $\mu \mathrm{g} / \mathrm{ml})$ in the presence and absence of LPS $(200 \mathrm{ng} / \mathrm{ml})$. Macrophages were also exposed classical and type II-inducing stimuli (Fig 5.3). After 8 h culture IL-10 (a), IL-12 (b), and IL-4 (c) were assessed via ELISA. IL-4 was measured in the absence of the treatments with un-opsonised SRBC and un-opsonised SEA. ${ }^{*} \mathrm{P}<0.05$ : One way ANOVA with Bonferroni post test; Conditions versus Classical. Data represents the mean+ SEM from duplicate wells of one experiment. NS represents $\mathrm{P}>0.05$. 


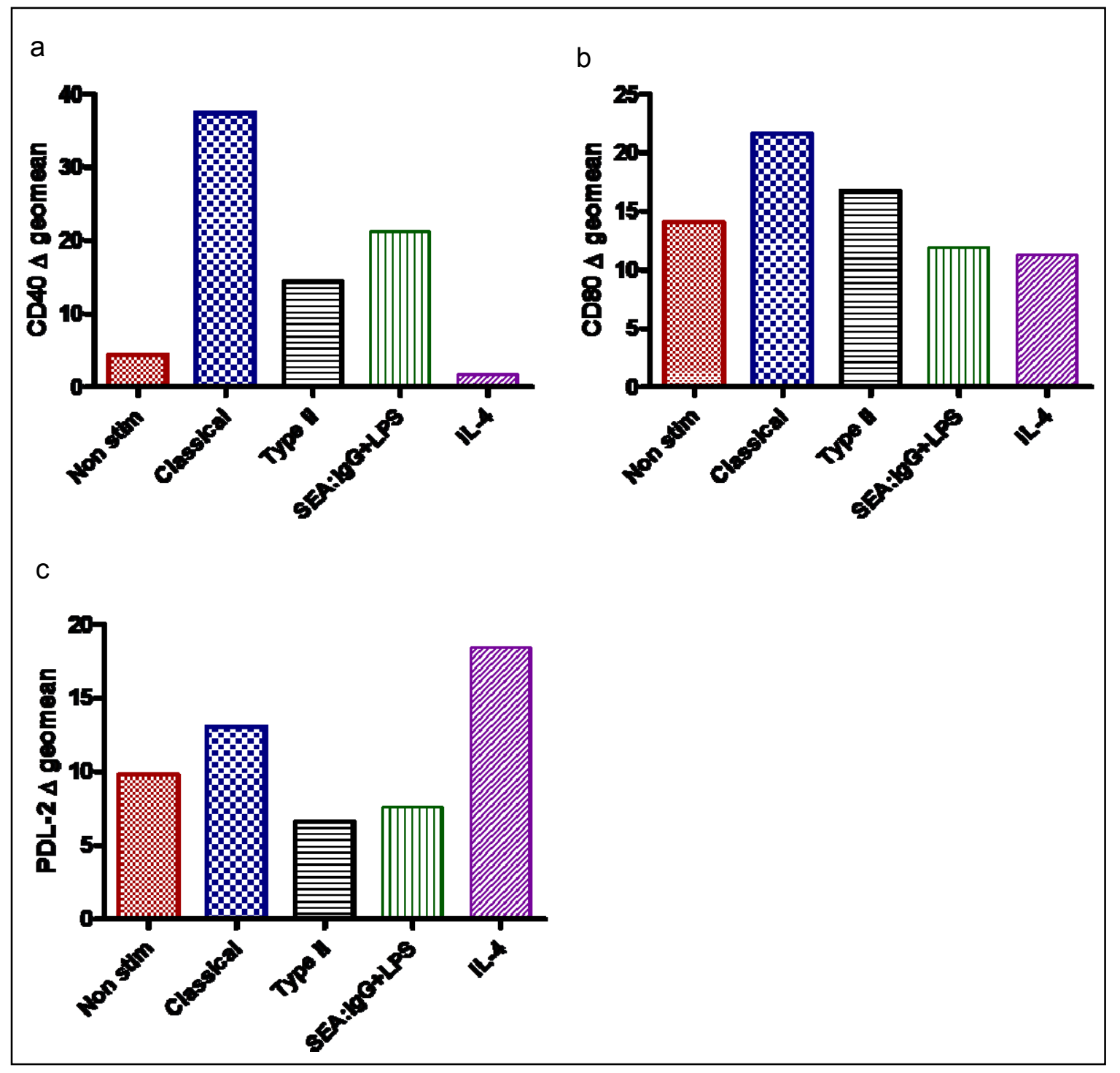

Figure 5.5 SEA complexes elicit similar alterations in surface markers to type II macrophages. Primary BMM $\varnothing\left(10^{6} \mathrm{cells} / \mathrm{ml}\right)$ derived from C57BL/6 mice were treated as mentioned in Figure 5.4. Additionally, macrophages were stimulated with $20 \mathrm{U} / \mathrm{ml}$ rlL-4. After $24 \mathrm{~h}$ culture CD40 (a), CD80 (b), and PDL-2 (c) were assessed via flow cytometry. Bars represent one experiment. 


\subsubsection{Enzymatic profile of macrophages exposed to SEA complexes}

In addition to cytokine production and surface marker expression, enzyme markers for classical, type II and alternative macrophage activation were investigated using western blotting to assess the activation state of macrophages induced by schistosome immune complexes plus LPS. Macrophages were stimulated for $9 \mathrm{~h}$ and $24 \mathrm{~h}$, and lysates were probed by western blotting for the expression of SPHK1 as a marker for type II activation, RELMa (FIZZ1) for alternative activation, and iNOS for classical activation. Alpha tubulin expression was used as a loading control and all samples were normalized to this protein. Similar to cytokine production, $9 \mathrm{~h}$ was found to be the optimal time to assess intracellular proteins of interest.

Representative gels and calculated band densities are shown in Figures 5.6 and 5.7 for iNOS, SPHK1, and RELMa. Band densities are presented as a percentage of control compared to LPS, in order to compare the expression of the various markers to classical stimulation. Graphs were generated from 2-4 western blots for most samples, although SEA complex plus LPS-stimulated macrophages (SEA:IgG+LPS) were only run once. Figure 5.6 shows a similar expression of iNOS in type II activated macrophages to that of classical macrophages. iNOS was not detected in samples stimulated with SEA alone, but was expressed in macrophages exposed to SEA or SEA complexes in the presence of LPS. Unfortunately the samples including the lysate from macrophages stimulated with SEA complex alone were unsuccessful, so the direct effect of this immune complex on macrophages cannot be assessed at this time. However, this condition will be an essential control to include in all future work based on this study. Taken together, these results indicate that SEA or SEA complex-activated macrophages in the presence of LPS, produce iNOS similarly to classical and type II activated macrophages.

To evaluate a type II-specific marker, the production of sphingosine-kinase 1 (SPHK1) was assessed in macrophages exposed to SEA complexes in the presence or absence of LPS and in type II activated macrophages. As expected, there was a significant increase in SPHK1 expression by type II activation in comparison to classical macrophages, further supporting its role as a marker for type II activation (Figure 5.6). Macrophages exposed to SEA alone or SEA in the presence of LPS exhibit higher SPHK1 expression than classical macrophages, but lower than that expressed in type II activated macrophages (Figure 5.6). The production of SPHK1 in response to SEA alone, indicated the SPHK1 expressed in response to SEA plus LPS, is primarily driven by the effect of SEA, and not necessarily by the addition of LPS, unlike iNOS expression. In contrast, there is no detectable SPHK1 expression in macrophages stimulated with SEA complexes plus LPS. However, as this condition was done only once, further work needs to be done to confirm this result. 
RELM $\alpha$ expression was also assessed as a marker for alternative activation which is known to occur during schistosome infection, and is induced by IL-4. As shown previously, RELM $\alpha$ expression in classical and type II activated macrophages was very low (Figure 5.7). In contrast, rIL-4-induced alternatively activated macrophages produced significant amounts of RELM $\alpha$, validating its high expression as a marker for alternatively activated macrophages. Additionally, SEA complexes in the presence of LPS induced much higher levels of RELM $\alpha$ compared to alternatively activated macrophages, suggesting that this condition may share some of the features of alternatively activated macrophages. Furthermore, given that IL-4 is produced by macrophages stimulated with SEA complexes and LPS, this elevation in RELM $\alpha$ is likely to be induced by this cytokine and not directly induced by the SEA complexes and LPS. Overall, the analysis of the enzymatic profile of macrophages indicates SEA either alone or in a complex is capable of inducing a distinct activation state from classical macrophages. The expression of markers for type II activation as well as alternative activation indicates SEA complexes are inducing the activation of macrophages into a 'hybrid' state, which exhibits characteristics from more than one known activation state. 


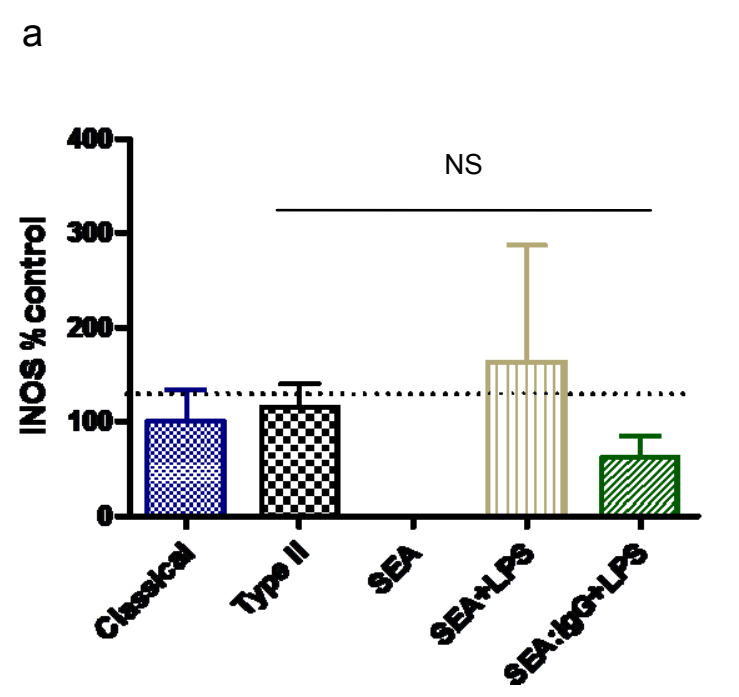

b

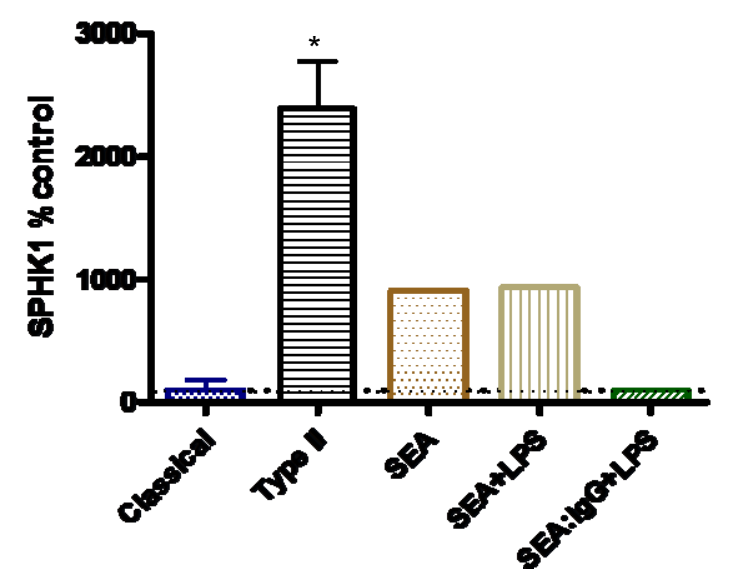

C

d

Media Classical TypeII $\wedge$ SEA $\wedge$ SEA+LPS Media Classical $\wedge$ SEA:IgG+LPS TypeII
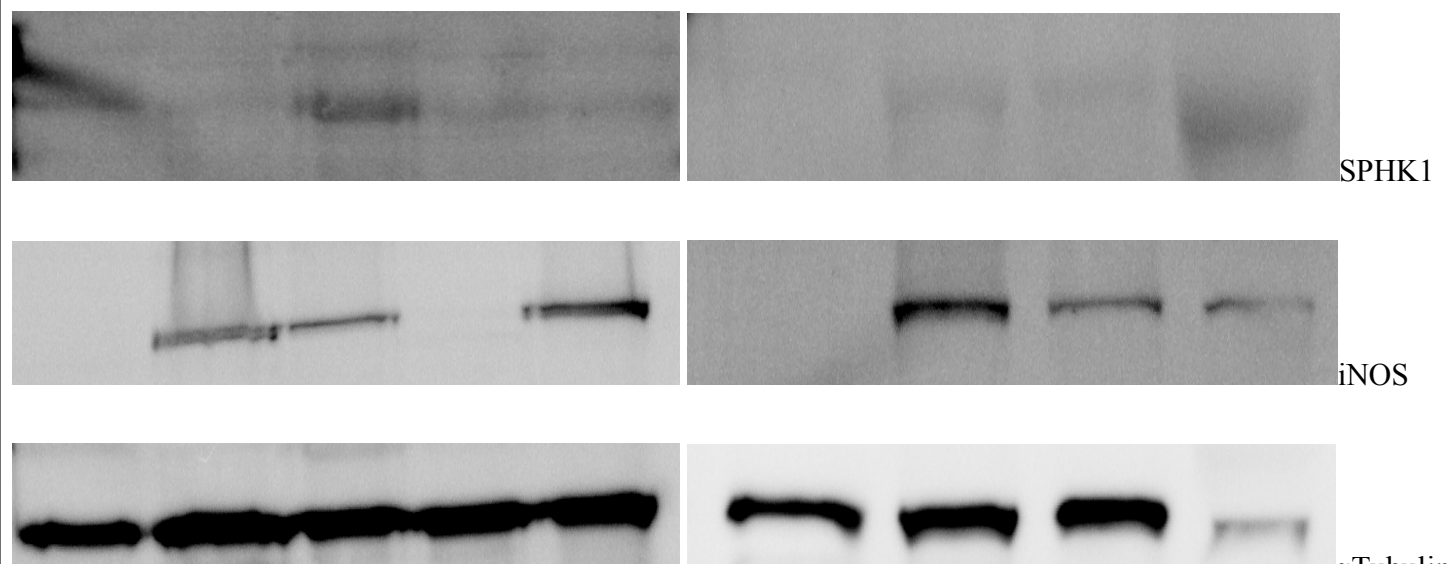

$\alpha$ Tubulin

Figure 5.6 SEA induces SPHK1 expression and iNOS in macrophages. Primary BMM $\varnothing\left(10^{6}\right.$ cells $/ \mathrm{ml}$ ) derived from C57BL/6 mice were primed with $20 \mathrm{U} / \mathrm{ml}$ IFN $\gamma$ followed exposure to $5 \mu \mathrm{g} / \mathrm{ml}$ SEA, or SEA-lgG in the presence or absence of $200 \mathrm{ng} / \mathrm{ml} \mathrm{LPS} \mathrm{(SEA-lgG:} 5 \mu \mathrm{g} / \mathrm{ml}-20 \mu \mathrm{g} / \mathrm{ml}$ ), or classical or type II-inducing stimuli. After $9 \mathrm{~h}$ culture, iNOS (a) and SPHK1 (b) were assessed via western blotting as mentioned in section 2.6, with anti-SPHK1 pAb (1:700), anti-iNOS pAb (1:700) and anti-atubulin (1:1000). Bars represent the band densities of 1-3 experiments normalised to atubulin. ${ }^{*} \mathrm{P}<0.05$ : One way ANOVA with Bonferroni post test; Conditions versus Classical activation (LPS). Bars for SEA:IgG + LPS represent a single experiment. Data is presented as \% of control compared to cultures with LPS. (c-d) are representative of gels used to elucidate (a-b). Differences between the gels are represented by $\left({ }^{\wedge}\right)$. Molecular weights: SPHK1 (69KDa), iNOS (135KDa), aTubulin (50KDa). 
a

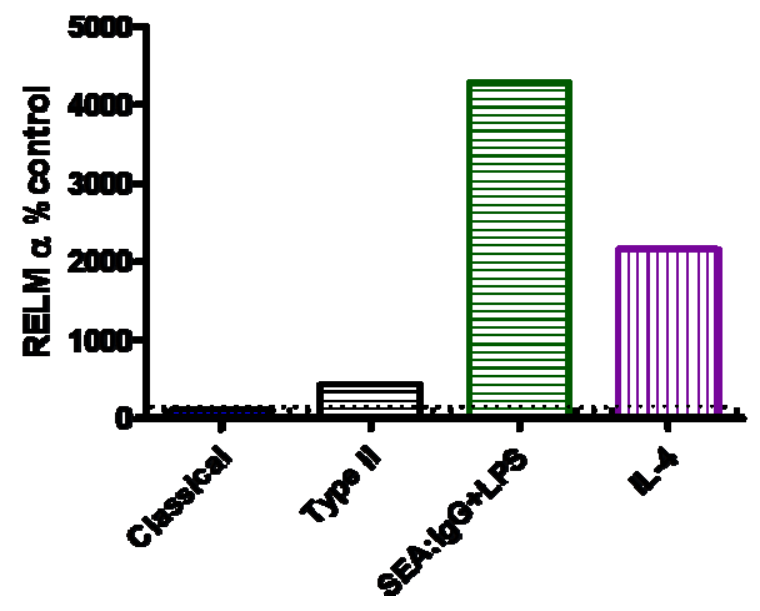

b

IL-4 SEA:IgG+LPS Type II $\quad$ Classical Media

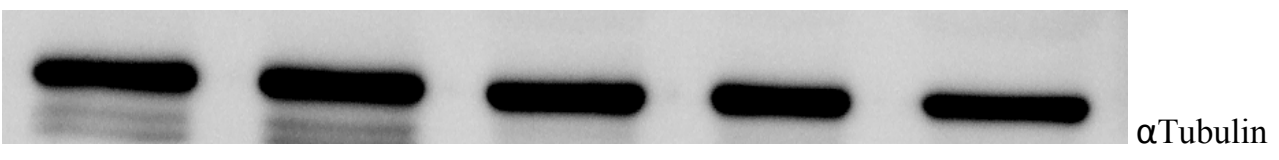

RELM $\alpha$

Figure 5.7 SEA complexes induce the expression of RELM $\alpha$ in macrophages. Primary BMM $\varnothing\left(10^{6}\right.$ cells $/ \mathrm{ml}$ ) derived from C57BL/6 mice were primed with $20 \mathrm{U} / \mathrm{ml} \mathrm{IFN} \gamma$ followed by exposure to SEAlgG + LPS $(5 \mu \mathrm{g} / \mathrm{ml}-20 \mu \mathrm{g} / \mathrm{ml}), 20 \mathrm{U} / \mathrm{ml} \mathrm{rlL}-4$, or classical or type II-inducing stimuli. After $9 \mathrm{~h}$ culture, RELMa was assessed via western blotting (section 2.6), with anti-RELMa (1:700) followed by anti-atubulin (1:1000). Bars represent the band densities of one experiment normalised to $\alpha$ tubulin. Data is presented as \% of control compared to cultures with LPS. Diagram (b) is the gel used to elucidate the graph in (a). Molecular weights: $\alpha$ Tubulin (50KDa), RELM $\alpha(12-15 \mathrm{KDa})$. 


\subsection{Discussion}

During infection with the parasitic helminth of the Schistosoma genera, a polarized Th2 response dominates in the host (Grzych et al., 1991; Pearce et al., 2004). Studies demonstrating an inverse correlation between areas prevalent in schistosome infection, and those prevalent in autoimmunities such as MS, has prompted researchers to hypothesise that schistosome infection may provide protection against MS (Fleming et al., 2007). Indeed, schistosome infection or exposure to isolated schistosome eggs or soluble egg antigen elicits protection against animal models of MS and type 1 diabetes (Cooke et al., 1999; La Flamme et al., 2003; Sewell et al., 2003; Zaccone et al., 2003; Zheng et al., 2008). The protection was accompanied by a reduction in Th1 inflammatory responses and an increase in Th2-type responses, and was abolished in the absence of this Th2 response (La Flamme et al., 2003; Sewell et al., 2003). In a similar manner, inducing type II macrophage activation in vivo protects from the animal model of MS in a Th2 dependent manner (Tierney et al., 2009). Therefore, this thesis investigated the macrophage response to schistosome egg or egg antigen immune complexes, in order to determine whether type II macrophage activation may be induced during schistosome infection and may thus contribute to the reported protective effect against autoimmune diseases.

The involvement of macrophages in driving protective Th2 responses to whole schistosome eggs is unknown; hence, we wished to identify the anti-inflammatory effects of exposure to schistosome eggs on macrophages. Furthermore, we assessed whether these egg immune complexes induced type II activated macrophages similar to those induced by SRBC complexes. We found that similar to opsonised SRBCs, schistosome egg and egg immune complexes, in the presence of LPS, significantly reduced IL-12 and increased IL-10 production in comparison to classically activated macrophages. However, unlike type II activation induced by opsonised SRBC, the effects seen with schistosome egg complexes were mirrored by the effects of schistosome egg in a non-complexed (i.e. non-opsonised) form. This result indicates the response is not specific to Fc $\gamma \mathrm{R}$ ligation as type II activation is (Lucas et al., 2005; Zhang et al., 2006a). However, macrophages activated in this manner by schistosome eggs may still be an important contributor to host protection due to their production of IL-10, given that IL-10 is essential for protection against severe disease caused by schistosomiasis (Hoffmann et al., 2000). Finally, previous work has clearly shown that the increased IL-10 in conjunction with reduced IL12 plays an important role in immune deviation and host protection during schistosomiasis (Hesse et al., 2004) and EAE (Sewell et al., 2003). Our findings indicate that schistosome egg either alone or in a complex with serum from a schistosome infected mouse has the ability to induce anti-inflammatory cytokine profiles similar to that observed with type II 
activation, however the mechanisms involved differ from that of type II macrophage activation.

Type II activation by opsonised SRBC induces a significant reduction in the expression of CD80, CD40 and PDL-1 in response to LPS (Tierney et al., 2009) (Chapter 3), but the effect of schistosome egg on the expression of these surface markers is currently unknown. Assessing the changes in CD80, PDL-1 and CD40 by schistosome eggs and egg complexes in the presence of LPS, we found the expression profile more similar to classical macrophages than that of type II activated macrophages. While there were slight reductions in the expression of CD40 and CD80 at the $24 \mathrm{~h}$ time-point, these reductions occurred independent of whether or not schistosome egg was in a complex form. Furthermore, the expression of PDL-1 in comparison to classical macrophages remained largely unchanged by stimulation with schistosome egg or egg complexes in the presence of LPS.

Thus, macrophages exposed to schistosome egg and egg complexes do not exhibit the same characteristics of type II activation in terms of the changes seen in activation marker expression. In addition to this, the similarity in response to the egg or egg complex suggests that the response induced by these products in the presence of LPS may be occurring through a different mechanism than that of $\mathrm{Fc} \gamma$ receptor ligation which evokes type II activation. It is more likely that components of the schistosome egg itself were eliciting the observed anti-inflammatory effects. These components may include glycosylated products (e.g. proteins, lipids) on the schistosome egg, which promote Th2 cell biasing (Okano et al., 1999). Schistosome eggs have been demonstrated to promote highly polarised Th2 immune responses, capable of protecting against EAE (Sewell et al., 2003), and this investigation further supports this finding by demonstrating that immune deviation can occur by direct interaction of the schistosome egg with macrophages. However, we have not shown that this effect on macrophages elicits Th2 cell biasing, but based on the cytokine profile, and previous in vivo observations (Sewell et al., 2003), it is highly possible. The ability of schistosome egg antigens, but not whole schistosome eggs, to induce Th2 biasing through the interaction with APCs has been previously reported (MacDonald et al., 2002c; Steinfelder et al., 2009). Dendritic cells exposed to a schistosome egg component, omega-1, altered their phenotype to elicit Th2 cell biasing upon contact with CD4 T cells (Steinfelder et al., 2009). It was concluded that the immune deviation was elicited by egg-induced changes in the level of signalling by DCs. Furthermore, in addition to the effects on DCs, the interaction of schistosome egg components with basophils has also been shown to induce immune deviation (Schramm et al., 2003) by up regulating IL-4, a strong inducer of Th2 development (Le Gros et al., 1990). The exact mechanism involved in deviating the macrophage response presented in 
this thesis is unknown; however further investigations using specific egg components like omega-1 should be carried out.

In contrast to whole schistosome eggs, the majority of published work focuses on schistosome soluble egg antigen (SEA), which is extremely antigenic and induces strong Th2 responses (Pearce et al., 2004; Zheng et al., 2008). Investigations have been carried out to elucidate the components of SEA which provide them with Th2-inducing properties, and some of the suggested components include IL-4 inducing principle of Schistosoma mansoni (IPSE) and N-fucopentaose III (Okano et al., 1999; Schramm et al., 2003), both of which induce highly polarised Th2 responses. Studies using SEA derived from Schistosoma japonicum have also shown that the SEA-specific Th2 responses are protective against EAE (Zheng et al., 2008). Because SEA immune complexes are generated during schistosomiasis (Rezende et al., 1997), it is possible that these complexes can promote type II activation as well as Th2 responses. Therefore, this investigation examined the effects of SEA complexes on macrophages in order to understand possible effects associated with prevention of inflammatory diseases like EAE.

Using the SEA immune complex, we found a significant increase in IL-10 and reduction in IL-12 in the presence of LPS. This finding complements in vivo data, in which protection against EAE by schistosome infection is associated with reductions in IL-12 and increases in IL-10 (La Flamme et al., 2003). It also correlates with the IL-10/IL-12 profile elicited by exposure to SEA complexed with serum (La Flamme et al., 2004), and the cytokine profile induced by type II activation as demonstrated in Chapter 3 . In contrast to SEA immune complexes, SEA and LPS had only a minor effect on cytokine production suggesting that, as with type II activation, the alternation in IL-10 and IL-12 occurred through ligation of Fc $\gamma$ Rs.

SEA has been demonstrated to contain components which strongly induce the production of IL-4 by immune cells such as basophils (Schramm et al., 2003). IL-4 production was assessed in the cultures of classically activated and type II activated macrophages, with very little IL-4 detected. In contrast to the nearly undetectable levels noted by classical and type II activated macrophages, macrophages stimulated with SEA complexes in the presence of LPS produced very high levels of IL-4. Although IL-4 cytokines are increased during schistosomiasis (Grzych et al., 1991), to date there have been no reports of macrophages producing IL-4 in response to schistosome infection or exposure to schistosome products. In fact, the only studies reporting the production of IL-4 by macrophages used the RAW 264.7 cell line derived from a BALB/c mice and alveolar macrophages during pulmonary fibrosis (Buttner et al., 1997; Mukherjee et al., 2009; Sempowski et al., 1994). Due to the important role of IL-4 in T cell biasing as well as in 
differentially activating alternatively activated macrophages and perpetuating antiinflammatory responses, the ability of SEA complexes to initiate IL-4 production merits further investigation as this pathway may be an important mechanism involved in driving Th2 responses.

Also investigated were the effects of SEA complexes on the expression of CD40, CD80, and PDL-2. In accordance with type II activation, SEA complexes in the presence of LPS induced a reduction in CD80 and CD40. Studies have previously demonstrated the requirement for CD40/CD40L signalling in the induction of Th2 responses (MacDonald et al., 2002c). As with type II activation, the reduction in CD40 but absence of complete inhibition in response to SEA complexes emphasizes the possible role of this particular level of CD40 signalling in promoting protective responses. As with type II activation, the reduction in the expression of these co-stimulatory markers was most significant in response to the complex form of SEA.

As IL-4 promotes alternative activation of macrophages (Raes et al., 2002; Stein et al., 1992), we assessed the expression of PDL-2, a ligand for PD-1 differentially up-regulated on alternatively activated macrophages (Loke et al., 2003; Tierney et al., 2009). Although rIL-4-induced alternatively activated macrophages induced PDL-2, neither type II activated nor SEA complex plus LPS-activated macrophages up-regulated PDL-2 expression. Given the significant increase in IL-4 by exposure to SEA immune complexes and LPS, this finding was contrary to expectation. It is unclear as to why there is such a difference between the effects of IL-4 derived from SEA-complex treated macrophages and rIL-4, which induced alternatively activated macrophages with respect to PDL-2 expression. This difference may be because the concentration of IL-4 produced by SEA complexactivated macrophages was below the threshold needed to drive all aspects of alternative macrophage activation or due to a difference in the mechanisms involved. The differences in the expression of PDL-2, CD80, CD40 and IL-12 by SEA complexes and LPS compared to rIL-4 indicate that these stimuli induce activation states with distinct profiles, whereas the SEA complex plus LPS condition follows a similar pattern of expression as type II activated macrophages.

In order to aid our understanding of the activation state induced by SEA complexes, the expression levels of enzyme markers for classical, type II and alternative macrophage activation were assessed. SPHK1 has been identified as an indicator of type II activation (Edwards et al., 2006), RELMa (FIZZ1) of alternative activation (Edwards et al., 2006; Raes et al., 2002) and iNOS production of classically and type II activated macrophages (Edwards et al., 2006). 
The similar production of iNOS by type II and classically activated macrophages corresponds to previous reports (Edwards et al., 2006), indicating both cell types are capable of eliciting immune responses against intracellular pathogens. This high expression of iNOS is in contrast to alternatively activated macrophages which do not express detectable levels of iNOS or NO but instead arginase 1 or RELMa (Modolell et al., 1995; Raes et al., 2002). If SEA complexes were inducing alternative activation, it would be expected that iNOS expression would be reduced or absent. However, iNOS was produced by macrophages exposed to both SEA and SEA complexes in the presence of LPS, but was not expressed under the same conditions in the absence of LPS. This indicates SEA or SEA complex-activated macrophages in the presence of LPS are likely capable of carrying out processes involved in intracellular killing, a mechanism associated with classical and type II activated macrophages (Campbell et al., 1996; Edwards et al., 2006), whereas in the absence of LPS, they are not.

In agreement with previous work (Edwards et al., 2006), SPHK1 expression was significantly higher in type II activated macrophages in comparison to classically activated macrophages. To date, there is no published data on the expression of SPHK1 by SEA or SEA complex treated macrophages; thus, the finding that there is an increase in SPHK1 expression in response to SEA compared to classically activated macrophages is a novel find. This SPHK1 expression was seen upon exposure to not only SEA alone, but also SEA in the presence of LPS, indicating SEA alone was driving this response rather than the presence of LPS. This is in contrast to the expression of iNOS which does depend on LPS exposure. It is important to note that the level of SPHK1 expression is less than that produced by type II activated macrophages, but much greater than any levels produced by classical macrophages. As such, its presence should not be used as a sole determinant of type II activation. It suggests the macrophages may be able to carry out similar functions to type II activated macrophages even if type II activation is not actually induced.

The role of SPHK1 in type II activation is currently unclear. Sphingosine-1-phosphate, produced via SPHK1, has been implicated to have an inhibitory role on Th1 cytokine production (Yang et al., 2005), but also in aiding survival of macrophages during proinflammatory stress (Wu et al., 2004). Recent studies have also indicated a role for sphingosine-1-phosphate in lymphocyte egress (Melendez, 2008), which may seem somewhat contradictory, but overall indicates a possible role in maintaining immune balance. The lack of expression of SPHK1 by SEA-complex and LPS-activated macrophages is an interesting finding as it does not follow the trend, in which SEA alone was seen to drive its production. This may indicate that when the SEA is in a complex form, the mechanism allowing SEA to induce SPHK1 expression is neutralised in some 
manner by the functional characteristic induced by SEA complexes. However, this action does not correlate with the other phenotypic characteristics induced by SEA complexes such as CD80/CD40/PDL-2 reductions and iNOS expression, which resemble those of type II activated macrophages. Therefore, further investigation in the effect of SEA complexes and LPS on SPHK1 production by macrophages is merited.

RELMa expression was assessed in order to elucidate whether alternative activation was occurring given the high production of IL-4 by SEA complex plus LPS-stimulated macrophages and the role of IL-4 in driving alternative activation (Stein et al., 1992). Type II activated macrophages produced nearly undetectable levels of RELMa, as did classically activated macrophages and this finding agrees with previous reports (Edwards et al., 2006). In contrast to classical and type II activation, macrophages exposed to recombinant IL-4 produced significant amounts of RELMa, corresponding with the induction of alternative activation (Raes et al., 2002). Thus, the appearance of high production of RELMa in response to SEA complexes in the presence of LPS, suggests the induction of alternative activation, with RELMa levels much higher than that expressed by macrophages exposed to even rlL-4.

Based solely on this western blot analysis, one would conclude that SEA complexes induced alternative activation of macrophages. However, this conclusion is not justified given the expression of SPHK1 and the cytokine profile, which suggest that neither type II nor alternative activation is induced. Altogether our findings indicate that SEA complexes induce a novel activation state exhibiting a hybrid of characteristics, blended from type II activation and alternative activation as well as some characteristics from neither state. Although 'hybrid' activation states have been previously suggested (Mosser et al., 2008), no induction of such a state as this has been previously reported.

The induction of this unique 'hybrid' activation state may have important beneficial implications to the regulation of autoimmunity as well as schistosomiasis. IL-4 is able to provide protection against EAE (Shaw et al., 1997) and schistosomiasis (Brunet et al., 1999), through the induction of Th2 responses. Understanding mechanisms involved in increasing IL-4 production may have extremely beneficial consequences in the treatment of inflammatory diseases. For example, RELMa aids wound repair (Raes et al., 2002) as well as suppresses excessive Th2 responses (Nair et al., 2009; Pesce et al., 2009b), and during schistosomiasis, RELMa is essential for the prevention of lethal levels of hepatic fibrosis induced by Th2 responses (Nair et al., 2009). Similarly, RELMa expression may also benefit EAE by aiding in repair of the CNS and preventing excessive $T$ cell responses. This thesis presents a novel activation state induced by schistosome egg antigen complexes and LPS, which exhibits a range of characteristics involved in 
maintaining a balance between the Th2 polarised fibrogenic responses required for host survival during schistosomiasis (Herbert et al., 2004), and reducing overzealous inflammatory responses which would otherwise induce pathology (Pesce et al., 2009b). Therefore this state of activation may be beneficial in EAE as well, where the balance of immune responses is essential. 


\section{Chapter 6: General discussion}

\subsection{Overall Summary of thesis}

Various studies have demonstrated the potent protective effects of immune deviation on inflammatory disorders (Tierney et al., 2009; Weber et al., 2007; Zheng et al., 2008). Macrophages are capable of deviating immune responses into pro-inflammatory or antiinflammatory responses dependant on their activation status (Edwards et al., 2006; Mosser et al., 2008a). In autoimmunites, studies have shown that protective immune deviation can be induced by pre-exposure to a Th2-inducing parasite Schistosoma mansoni, as well as pre-treatment with type II activated macrophages which elicit Th2 biasing (La Flamme et al., 2003; Sewell et al., 2003; Tierney et al., 2009). The exact mechanisms involved in type II activation and the induction of protective anti-inflammatory responses during schistosomiasis are unknown and currently under investigation. Similarities exist between the effects of type II activated macrophages and schistosome infection in protection against an animal model of MS, EAE. It is unknown what effect schistosome infection has on macrophage activation, however high levels of circulating immune complexes as well as inflammatory mediators occur during infection (Rezende et al., 1997; Santoro et al., 1979), making it likely that type II activation is a contributing factor in the protection schistosomiasis elicits. Understanding the immunological events involved in protection by either type II activation or other macrophage phenotypes which may be induced during schistosomiasis, may benefit the development of drugs which mimic or enhance the protective effects against autoimmune diseases.

This thesis investigates mechanisms involved in perpetuating the phenotypic profile of type II activated macrophages, and promoting the deviation of $\mathrm{T}$ cell responses into polarised Th2 responses, with particular emphasis on IL-10 and CD40. This investigation also encompasses the effects of schistosome immune complexes on macrophage activation, in order to elucidate whether type II activation may be induced in the course of schistosome infection.

\section{A 'hybrid' activation state}

Although results from the schistosome investigations are preliminary and require validation, they provide new insight into possible effects of schistosome infection on macrophage activation. Mosser et al reported the notion that some macrophages elicit traits of more than one activation state, termed 'hybrid macrophages' (Mosser et al., 2008b). To my knowledge, there are been no reports to date which have induced such a state. However this investigation may have identified such a 'hybrid' state, induced by exposure to schistosome egg antigen complexes. By only looking at surface markers, the 
SPHK1 enzyme, and cytokines IL-10 and IL-12, it would suggest schistosome complexes induce type II macrophage activation. However, the high levels of RELMa (a marker of alternative activation (Raes et al., 2002)) and high IL-4 production (a characteristic unknown of any activation states) make this macrophage phenotype distinct from both type II activation and alternative activation. The high production of IL-4 was a novel find, as to date the only reports of IL-4 production by macrophages include alveolar macrophages (Buttner et al., 1997; Sempowski et al., 1994) and macrophage cell lines (Mukherjee et al., 2009).

This activation state may elicit more beneficial effects compared to either type II or alternative activation states alone. The high levels of IL-4 and IL-10 and reduced IL-12 may induce anti-inflammatory immune responses, promoting the development of Th2 and Treg cells as has been previously reported by this cytokine environment (Le Gros et al., 1990; Roncarolo et al., 2008; Skapenko et al., 2005), while inducing the activation of other innate cells into regulatory states (Mantovani et al., 2007). Furthermore, this macrophage phenotype may pose as one of the main initiators of Th2 responses, as the initial source of IL-4 during schistosome infection has previously been questioned. Understanding mechanisms involved in IL-4 production will be extremely beneficial in the context of inflammatory illnesses. IL-4, as opposed to IL-12, is associated with protection from EAE (Shaw et al 1997). Through the expression of RELMa and SPHK1, this activation state may be beneficial in both wound healing (Raes et al., 2002) as well as suppression of overzealous T cell responses (Nair et al., 2009; Pesce et al., 2009). These characteristics may therefore be beneficial in protection against severe schistosomiasis as well as $\mathrm{EAE}$ and other pro-inflammatory diseases which require immunoregulation.

\section{Features of anti-inflammatory macrophages}

Classically activated macrophages, IFN $\gamma$ producing Th1 cells and IL-23-induced Th17 cells are implicated as major cell types associated with the pathogenesis of EAE (Cua et al., 2003; Langrish et al., 2005; O'Connor et al., 2008; Tran et al., 1998). Conditions which alter the immune response and establish an anti-inflammatory environment to prevent the accumulation of inflammatory cells, protect against EAE. This has been demonstrated in mice, by the administration of type II activated macrophages or pre-infection with parasites of the Schistosoma spp., which deviate the immune response into a polarised Th2/Treg/anti-inflammatory status and prevent the initiation of inflammatory disease (Sewell et al., 2003; Tierney et al., 2009). Only minimal information on the mechanisms of protection provided by schistosome infection or type II macrophages is known, therefore we investigated a broader range of effects of type II activation in order to expand our current knowledge, and to gain a greater understanding of mechanisms of action. 
Type II activation reduced the production of the chemokine MCP-1 in an inflammatory environment, as well as IL-6. High levels of IL-6 and MCP-1 are attributed to disease progression (Maimone et al., 1997; McManus et al., 1998), and inhibition of MCP-1 as well as IL-6 have proven successful in preventing the infiltration of inflammatory cells into the CNS (Huang et al., 2001; Serada et al., 2008). Although this investigation, in accordance with previous literature, demonstrated the suppressive effects of IL-10 on the expression of pro-inflammatory cytokines and chemokines (Anderson et al., 2002; Bogdan et al., 1991), we found IL-10 was not responsible for the anti-inflammatory profile elicited by type II activation. This has been previously demonstrated by Anderson et al, but only with regards to IL-12 reductions. Our investigations support that of Anderson et al, while further illustrating that reductions in the expression of co-stimulatory markers, and inflammatory mediators MCP- 1 and IL- 6 by type II activation, occur independently of IL10. This implies the existence of a novel mechanism whereby selective pro-inflammatory responses are reduced by $\mathrm{Fc} \gamma$ receptor ligation. Also demonstrated here is the broader effect of type II activation on chemokine and cytokine production. Although previous studies (Anderson et al., 2002; Gerber et al., 2001; Grazia Cappiello et al., 2001) have concluded type II activation has no effect on other pro-inflammatory cytokines such as IL6 and TGF $\beta$, the observations made were at the mRNA level, and not the secreted protein. This is the first study to my knowledge where a reduction in MCP-1 and IL-6 protein by type II activation is demonstrated, illustrating additional mechanisms other than through reduced IL-12, which may aid in protection against EAE.

As with CD40 reductions, another shared characteristic of anti-inflammatory phenotypes induced by type II activation or exposure to schistosome complexes, was the expression of spingosine kinase 1 (SPHK1), an enzyme whose activity in vivo is still controversial. SPHK1 is expressed in a variety of cells including T cells, mast cells, and basophils (Kihara et al., 2006; Yang et al., 2005). However, its significant up-regulation in macrophages has so far only been attributed to type II activation (Edwards et al., 2006). In this investigation, we reported a significant increase in this enzyme in response to schistosome immune complexes. Our investigations, as well as previous literature, illustrate the anti-inflammatory profile of macrophages elicited by exposure to schistosome immune complexes as well as type II-inducing stimuli, with both implicated in the biasing of T cell responses into the Th2 subset (MacDonald et al., 2002b; Okano et al., 1999; Zheng et al., 2008). Therefore, it is possible that in the context of the macrophages, SPHK1 supports functions involved in promoting Th2 development. SPHK1 activation in Th1 cells has demonstrated a role in reducing Th1 associated cytokines IFN $\gamma$ and IL-2 (Yang et al., 2005), therefore it is possible that similar effects occur, caused by macrophage-derived SPHK1. Humans and mice express three isoforms of SPHK1, all of 
which exhibit slight variations in their catalytic activities (Billich et al., 2003; Kihara et al., 2006). Therefore, it is possible that some of the conflicting results observed in vivo, such as its' role in promoting inflammatory responses and cell migration (Melendez, 2008), were due to the activity of the different isoforms under different environmental conditions, as well as the level, location and source of SPHK1. To date, the exact role of SPHK1 in macrophages remains elusive, especially with regards to type II activated macrophages. As SPHK1 is expressed in type II activated macrophages as well as macrophages activated into the 'novel' state by exposure to schistosome complexes, it seems likely that SPHK1 contributes in some way to the anti-inflammatory outcome, and therefore warrants further investigation.

These investigations also illustrated a reduction in CD40 associated with increased antiinflammatory functions. Supporting this notion is the reduction in LPS-induced CD40 expression which occurred in response to both schistosome immune complexes and SRBC immune complexes. Studies have shown that an active component of schistosome egg antigen (SEA), Omega-1, significantly increases Th2 responses and reduces Th1 development, with a reduction in CD40 expression on dendritic cells observed (Steinfelder et al., 2009). However, the investigators did not assess the significance of this reduction at the time. As with Steinfelder et al, we saw a reduction in CD40 in response to schistosome immune complexes as well as type II-inducing stimuli on macrophages, as opposed to dendritic cells. CD40 promotes the production of inflammatory cytokines such as IL-6, IL-12 and IL-23, all of which are associated with the induction of pathologyinducing Th1 and Th17 subset development (Langrish et al., 2005; Perona-Wright et al., 2009). In mice deficient in CD40, a significant inhibition of Th17 and Th1 development is noted, through the reduction of IL-6, IL-23 and IL-12 production, which therefore protects against EAE (Perona-Wright et al., 2009). The reduction in CD40 on macrophages exposed to type II-inducing stimuli or schistosome complexes, may therefore significantly impair the development of Th17 cells through the reductions in inflammatory cytokines, as well as reducing the infiltration of inflammatory cells through reduced chemokine production. Therefore, this investigation puts into focus another possible mechanism contributing to the protection type II activation and schistosome infection provide against EAE.

\section{Involvement of CD40 signalling in T cell biasing}

Although CD40 expression has long been associated with Th1-driven cell mediated responses (Alderson et al., 1993; Campbell et al., 1996), studies in 2002 by MacDonald et al illustrated the essential requirement of CD40 signalling for the induction of Th2 responses by dendritic cells. Type II activation or schistosome infection protect against EAE (La Flamme et al., 2003; Sewell et al., 2003; Tierney et al., 2009), and as 
demonstrated in this thesis, macrophages express reduced CD40 in the absence of complete inhibition upon exposure to either stimuli. Furthermore, both are capable of polarising Th2 responses (Gerber et al., 2001; La Flamme et al., 2006; Zheng et al., 2008). This suggests to us that low but maintained levels of CD40 on macrophages (i.e. not completely abolished) have a role in deviating $T$ cell responses. This thesis therefore assessed the effect of altered levels of CD40/CD40L signalling between $T$ cells and macrophages, with the aim of identifying if there was a particular low level which enhances Th2 cell biasing.

Although we did not enhance type II activation through the inhibition of CD40/CD40L signalling, we did see significant differences in $\mathrm{T}$ cell responses. These differences depended on the level of CD40/CD40L signalling. An example was the loss of IL-10 suppression with $\mathrm{T}$ cells that were exposed to classical macrophages after the signalling was reduced. Furthermore, low levels of CD40/CD40L inhibition had no significant effect on Th2 polarisation by type II activation, while Th1 development was still significantly impaired. An inhibitory effect on both Th2 and Th1 responses was seen in the complete absence of CD40/CD40L signalling however. Further studies may be required; however this investigation suggests that at low levels of CD4OL signalling, Th1 development is reduced, whereas there is little effect on the responsiveness of $\mathrm{T}$ cells to Th2-inducing stimuli by type II activation. In the setting of inflammatory illnesses, macrophages which elicit this low level of CD40/CD40L interaction seen by type II macrophages may be beneficial in depressing detrimental Th1 responses while maintaining protective Th2 development. This would somewhat explain why in some circumstances the absence of CD40 results in the ablation of Th2 development (MacDonald et al., 2002b), whereas in other situations, CD40L inhibition protects against disease by increasing Th2 development, as seen in the prevention of graft rejection (Hancock et al., 1996). This may be a result of the preferential inhibition of Th1 and not Th2 responses, when there is incomplete blockage of CD40L.

Major producers of IL-10 include Th2 cells (Sornasse et al., 1996) as well as Tregs (Groux et al., 1997). Although IL-2Ra and IL-4Ra expression are commonly associated with the induction of Th2 responses (Cote-Sierra et al., 2004), it is also possible that type II activated macrophages induce Tregs. Studies have shown IL-4 to induce Tregs from naive CD4 T cells, resulting in high expression of IL-2Ra (CD25) and increased IL-10 (Skapenko et al., 2005), which are characteristics seen in our studies. Furthermore, McKee et al demonstrated the role of both Tregs and Th2 cells in the suppression of Th1 development during schistosomiasis (McKee et al., 2004), illustrating the possible effect of schistosome complexes or type II activation on Treg development. Further investigations would be required to assess this induction, however as proliferation was not inhibited by $T$ 
cells exposed to type II macrophages, it may be fair to assume the responses were that of a Th2 polarized subset.

Our results support previous reports (MacDonald et al., 2002b), whereby the complete absence of CD40 results in a reduction in the development of Th2 cells, as well as supporting the notion that differential levels of CD40 signalling alter counter-regulatory responses (Mathur et al., 2004). Also provided in this thesis are some novel insights into the suppressive effects of CD40/CD40L signalling on IL-10 production, a mechanism which may be a contributing factor to the suppression of both Th1 and Th2 responses seen at high levels of CD40 inhibition, and may possibly be involved in the induction of suppressive regulatory T cells (Wan et al., 2009).

\subsection{Summary}

Further supporting previous literature (Mathur et al., 2004; Murugaiyan et al., 2006), this thesis illustrates the intricate balance a single co-signalling molecule can elicit. As with MacDonald et al, CD40 was found essential for the induction of Th2 responses, which in our setting was elicited via type II activation. IL-10, although suppressive of IL-12 as also previously reported (D'Andrea et al., 1993), was not required for the cytokine and surface marker profile elicited by type II activation. This supports studies by Grazia Cappiello et al, who saw no effect in the ability of type II macrophages to reduce IL-12, while extending the knowledge to the effects on other cytokines/chemokines and surface markers which were not previously investigated. As well as providing further insight into alterations type II activation induces at both the level of macrophages and $\mathrm{T}$ cells, we present previously unreported effects of schistosome complexes on macrophage activation, illustrating the induction of what seems to be a novel activation state exhibiting a hybrid of characteristics of type II and alternative activation. The findings from this thesis bring us a step closer to understanding mechanisms involved in promoting anti-inflammatory immune responses, which is essential for identifying more effective treatments for autoimmunities. Furthermore, although much remains to be validated, provided here are some new avenues of research for future investigations into understanding essential components of immune deviation.

\subsection{Future}

Future studies may involve investigating the roles of SPHK1, a shared marker of both type II activation and the 'hybrid' state induced by schistosome immune complexes. As both type II activation and schistosome infection protect against EAE, SPHK1 and RELMa may be important contributors to this effect (La Flamme et al., 2003; Sewell et al., 2003; Tierney et al., 2009; Zheng et al., 2008). Investigations may also be carried out to elucidate whether the activation state induced by schistosome complexes in vitro, occurs 
in vivo, and whether it contributes to protection against EAE. As T cell proliferation in response to type II activation and classical activation were similar despite the reduction in PDL-1 on type II macrophages, further investigations are required to elucidate the role of PDL-1/PD-1 signalling during type II activation. This co-signalling interaction, as with CD40L signalling, may be required for Th2 cell biasing. Further understanding the effect of co-signalling molecules on $\mathrm{T}$ cell biasing may have important implications in the treatments of not only pro-inflammatory associated diseases, but also those diseases such as leishmaniasis which require efficient Th1 responses (Stenger et al., 1996). The suppressive effect of CD40/CD40L signalling on anti-inflammatory cytokines such as IL-10 should also be further investigated, as should the effects of increasing CD40 signalling on $\mathrm{T}$ cell responses in contrast to CD40 inhibition. Although CD40 inhibition was unable to increase the anti-inflammatory Th2-biasing capabilities, it would still be interesting to investigate the outcomes of different levels of increased CD40/CD40L signalling.

Identifying ways to enhance anti-inflammatory responses against EAE will not only benefit the development of treatments for MS, but will also have positive implications in the treatment of other inflammatory illnesses. Furthermore, the increase in knowledge about mechanisms involved in different macrophage phenotypes will enable us to better identify mechanisms involved in promoting or preventing diseases. 


\section{Appendix A-Supplementary Tables and figures}

TableA1 Final concentrations/dilutions of ELISA reagents

IL-12p40 (BD Biosciences)

\begin{tabular}{|l|l|l|}
\hline Reagent & Dilution/concentration & Diluent \\
\hline Capture & $1: 1000$ & $0.1 \mathrm{M} \mathrm{Na}_{2} \mathrm{HPO}_{4}, \mathrm{pH}=9.0$ \\
\hline Standards & $4 \mathrm{ng} / \mathrm{ml}$ & $5 \% \mathrm{FCS}$ in $\mathrm{PBS} \mathrm{pH}=7.4$ \\
\hline Detection & $1: 1000$ & $5 \%$ FCS in $\mathrm{PBS} \mathrm{pH}=7.4$ \\
\hline SA-HRP & $1: 2000$ & $5 \%$ FCS in $\mathrm{PBS} \mathrm{pH}=7.4$ \\
\hline
\end{tabular}

\section{IL-10 (BD Biosciences)}

\begin{tabular}{|l|l|l|}
\hline Reagent & Dilution/concentration & Diluent \\
\hline Capture & $1: 500$ & $0.1 \mathrm{M} \mathrm{Na}_{2} \mathrm{HPO}_{4}, \mathrm{pH}=9.0$ \\
\hline Standards & $25 \mathrm{ng} / \mathrm{ml}$ & $10 \% \mathrm{FCS}$ in $\mathrm{PBS} \mathrm{pH}=7.4$ \\
\hline Detection & $1: 2500$ & $10 \% \mathrm{FCS}$ in PBS pH=7.4 \\
\hline SA-HRP & $1: 1000$ & $10 \%$ FCS in PBS pH=7.4 \\
\hline
\end{tabular}

IFN $\gamma$ (BD Biosciences)

\begin{tabular}{|l|l|l|}
\hline Reagent & Dilution/concentration & Diluent \\
\hline Capture & $1: 1000$ & $0.1 \mathrm{M} \mathrm{Na}_{2} \mathrm{HPO}_{4}, \mathrm{pH}=9.0$ \\
\hline Standards & $4 \mathrm{ng} / \mathrm{ml}$ & $5 \%$ FCS in $\mathrm{PBS} \mathrm{pH}=7.4$ \\
\hline Detection & $1: 4000$ & $5 \%$ FCS in PBS $\mathrm{pH}=7.4$ \\
\hline SA-HRP & $1: 2000$ & $5 \%$ FCS in $\mathrm{PBS} \mathrm{pH}=7.4$ \\
\hline
\end{tabular}


IL-4 (BD Biosciences)

\begin{tabular}{|l|l|l|}
\hline Reagent & Dilution/concentration & Diluent \\
\hline Capture & $1: 2000$ & PBS, $\mathrm{pH}=7.4$ \\
\hline Standards & $50 \mathrm{U} / \mathrm{ml}$ & $5 \%$ FCS in PBS pH=7.4 \\
\hline Detection & $1: 4000$ & $5 \%$ FCS in PBS $\mathrm{pH}=7.4$ \\
\hline SA-HRP & $1: 2000$ & $5 \%$ FCS in PBS $\mathrm{pH}=7.4$ \\
\hline
\end{tabular}

Table A2 Western blot antibody dilutions

\begin{tabular}{|l|l|l|}
\hline Primary Antibody & Company & Dilution \\
\hline Anti-tubulin-Rabbit & Abcam & $1: 700$ \\
\hline Anti-SPHK1-Rabbit & Abcam & $1: 700$ \\
\hline Anti-RELMa-Rabbit & Abcam & $1: 700$ \\
\hline Anti-iNOS-Mouse & Transduction Laboratories & $1: 700$ \\
\hline
\end{tabular}

Dilutions of primary antibodies made in $1.5-2 \mathrm{ml}$ of $5 \%$ blocking solution (5\% non-fat skim milk in TTBS)

\begin{tabular}{|l|l|l|}
\hline Secondary Antibody & Company & Dilution \\
\hline Anti-Rabbit cy3 & GE Healthcare & $1: 1000$ \\
\hline Anti-Mouse cy5 & GE Healthcare & $1: 660$ \\
\hline
\end{tabular}

Dilutions of secondary antibodies made in 1.5-2mls of TTBS (appendix B) 
Kinetic graphs for type II activation, with additional controls for the absence of LPS

a

$\cos$

b

PDL-1
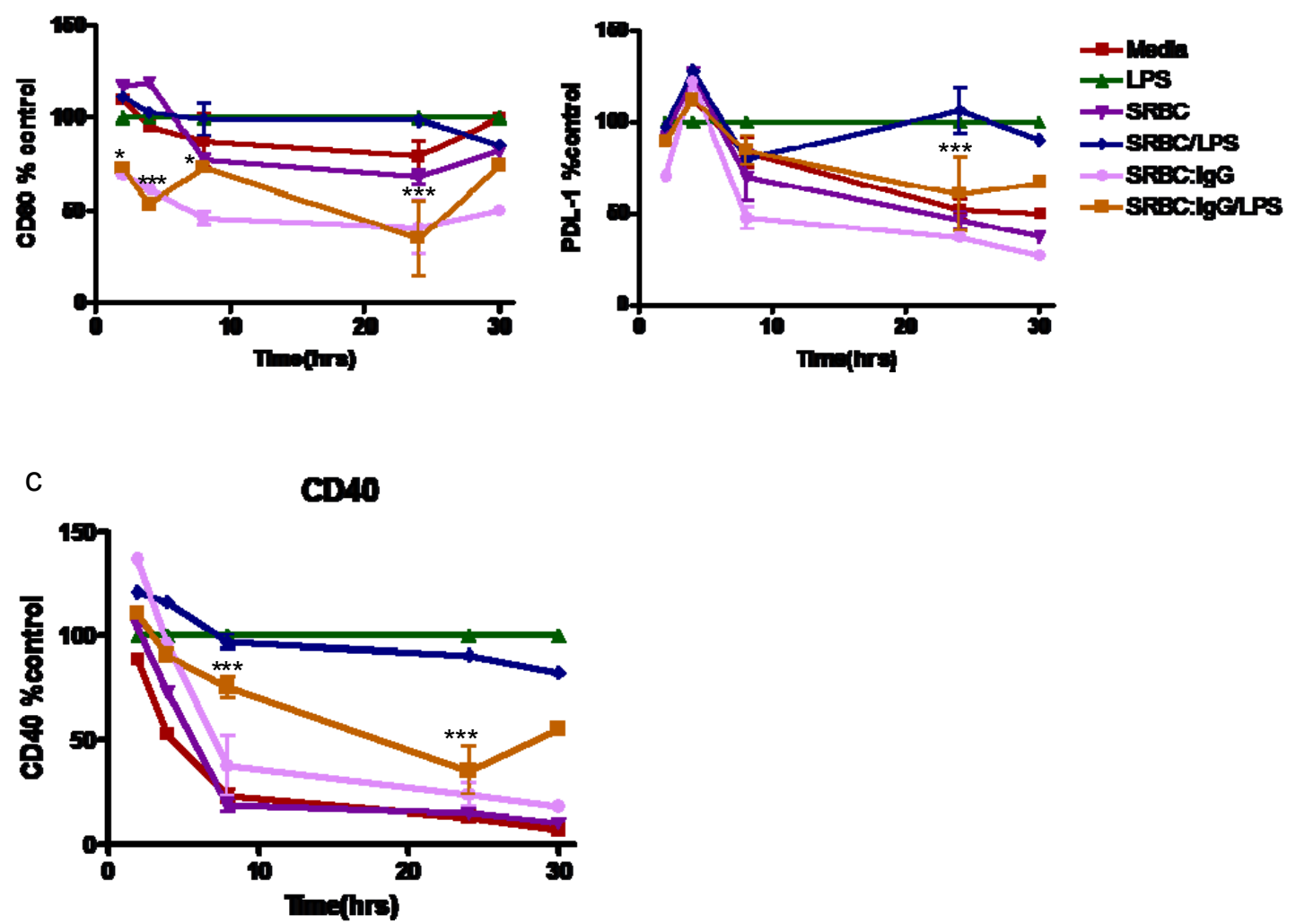

FigA1 Macrophages exposed to type II-inducing stimuli exhibit reductions in co-signaling molecules maximally at $24 \mathrm{~h}$ post stimulation: Macrophages derived from C57BL/6 mice were stimulated with $200 \mathrm{ng} / \mathrm{ml}$ LPS alone or LPS in the presence of SRBC or opsonised SRBC (SRBC:IgG). Cells were also left non-stimulated in media or cultured with SRBC or SRBC-IgG in the absence of LPS. After 2, 4, 8, 24, or $30 \mathrm{~h}$ of culture, CD80 (a), PDL-1 (b), and CD40 (c) were assessed via flow cytometry. ${ }^{*} \mathrm{P}<0.05,{ }^{* *} \mathrm{P}<0.01,{ }^{* * *} \mathrm{P}<0.001$ : Two way ANOVA with Bonferoni post test : SRBC:IgG+LPS vs LPS. Data points represent the mean +S EM of two experiments. 
Effects of CD40 signaling on macrophage phenotype- 24 hour time point (as opposed to the presented 8 hour time point).

a

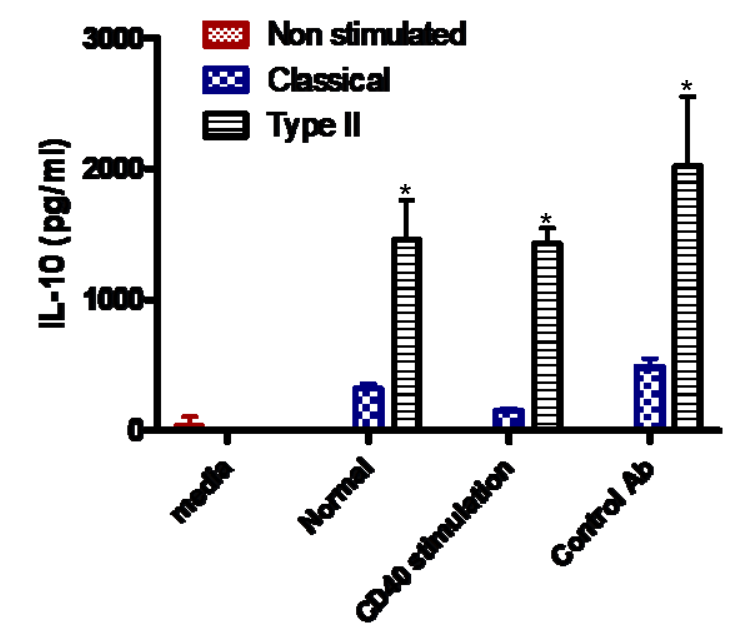

b

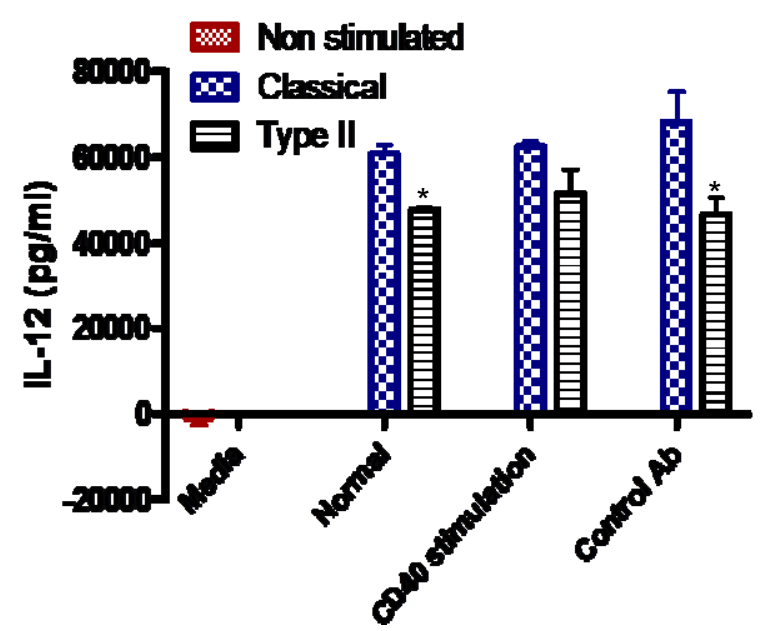

Figure A2 CD40 stimulation does not effect the IL-10/IL-12 ratio between classical and type II macrophages at $24 \mathrm{~h}$ after stimulation Macrophages $\left(10^{6} \mathrm{cell} / \mathrm{ml}\right)$ were exposed to classical or type II activating stimuli. Stimulatory rat-anti-mouse CD40 antibody (3/23) and IgG isotype control (rat $\mathrm{lgG}$ ) $(8 \mu \mathrm{g} / \mathrm{ml})$ (BD Bioscience) were added to appropriate cultures at the time of primary stimulation. After $24 \mathrm{~h}$ culture IL-10 and IL-12 were assessed via ELISA. ${ }^{*} \mathrm{P}<0.05$ two way ANOVA with Bonferroni post-test; Type II versus Classical. Bars represent datapoints (mean+SEM) of duplicate wells. 
Graphs on CD40/CD40L alterations, with data from co-cultures with nonactivated macrophages: Supports the notion that remaining IFN $\gamma$ (From overnight priming) in co-cultures elicited $\mathrm{T}$ cell responses in the absence of stimulants.

a

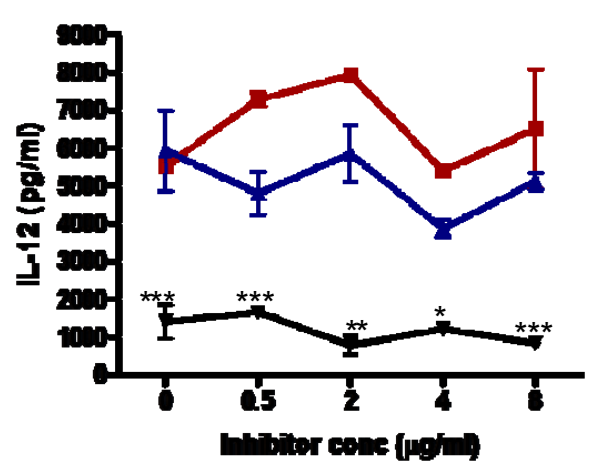

b

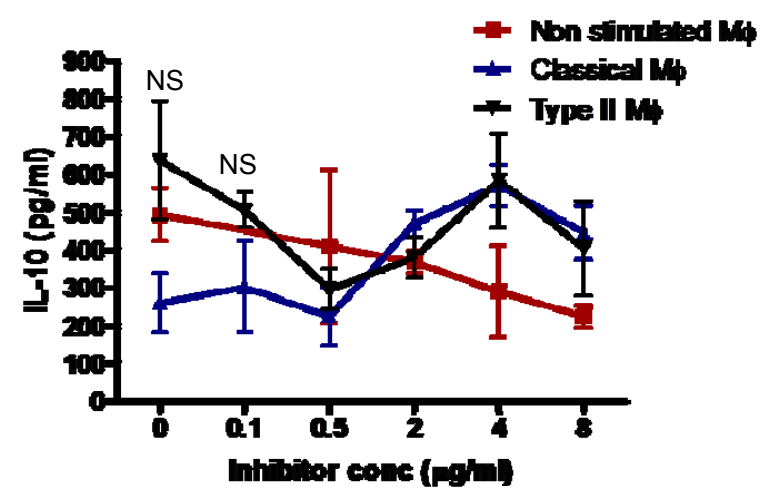

C

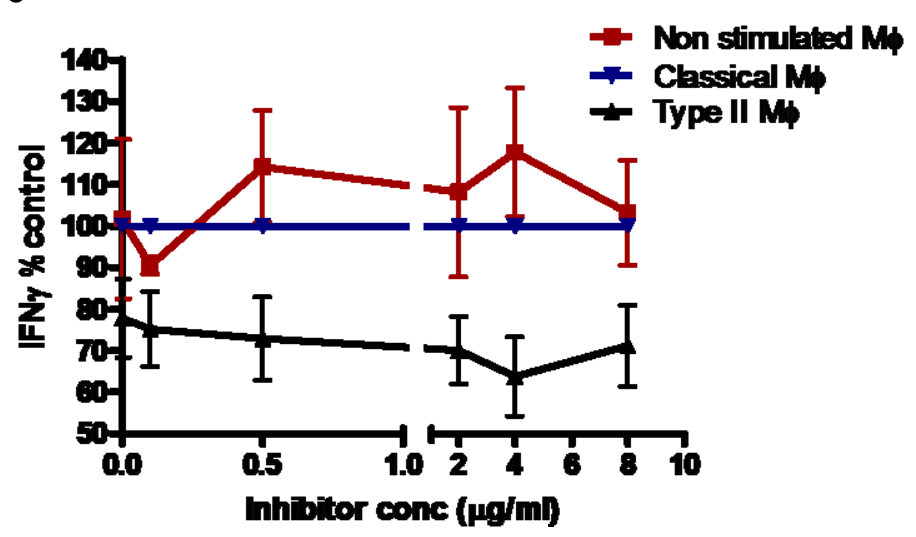

Figure A3 $\mathrm{CD} 40 / \mathrm{CD} 40 \mathrm{~L}$ inhibition has no effect on IL-12 or IFN $\gamma$ suppression by type II macrophages but alters IL-10 production Macrophages $\left(10^{6} \mathrm{cells} / \mathrm{ml}\right)$ were exposed to classical or type II activating stimuli. CD4 T cells were isolated from OTII mice and stained with CFSE. CD4 T cells $\left(2.5 \times 10^{6} \mathrm{cells} / \mathrm{ml}\right)$ plus $1 \mu \mathrm{M} \mathrm{OVA}_{323-339}$ peptide was added to macrophage cultures, along with varying concentrations (0.1-8 $\mu \mathrm{g} / \mathrm{ml})$ of anti-mouse CD40 ligand antibody (MR1) $3 \mathrm{~h}$ after classical or type II activation. After $72 \mathrm{~h}$ culture IL-12 (a), IL-10 (b), and IFN $\gamma$ (c) were assessed via ELISA. ${ }^{*} \mathrm{P}<0.05{ }^{* *} \mathrm{P}<0.01{ }^{* * *} \mathrm{P}<0.001$ : Two way ANOVA with Bonferroni post test ; responses to Type II vs Classical $M \varnothing$. Graphs represent datapoints (mean + SEM) from two experiments. (c) is presented as $\%$ of control compared to cultures with classical macrophages. 


\section{Kinetic graphs for schistosome egg exposure with additional controls in the absence of LPS}

a

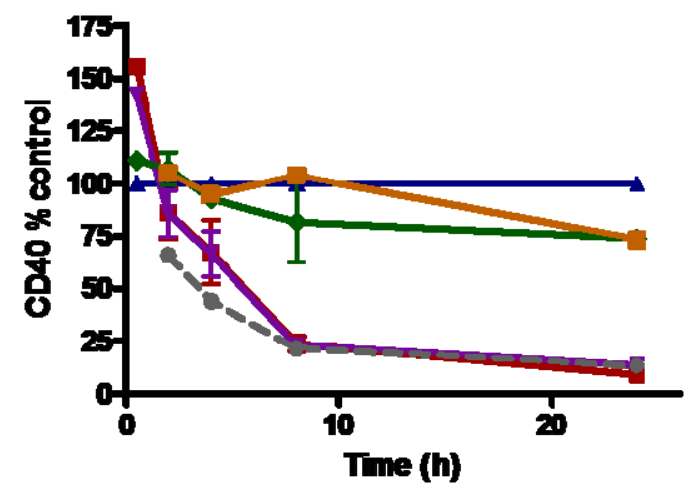

C

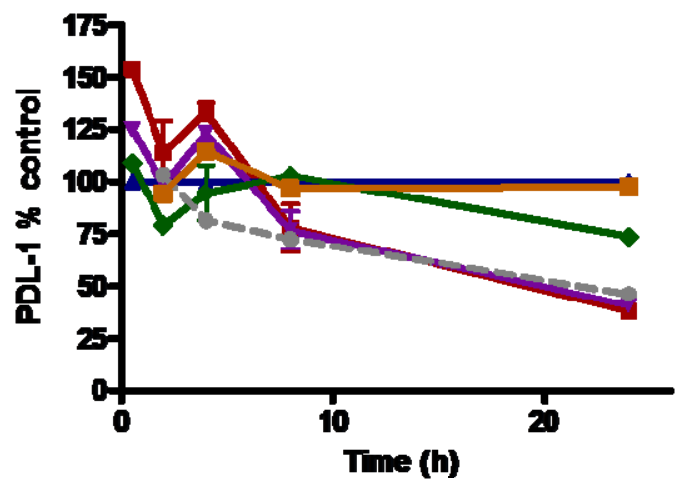

b

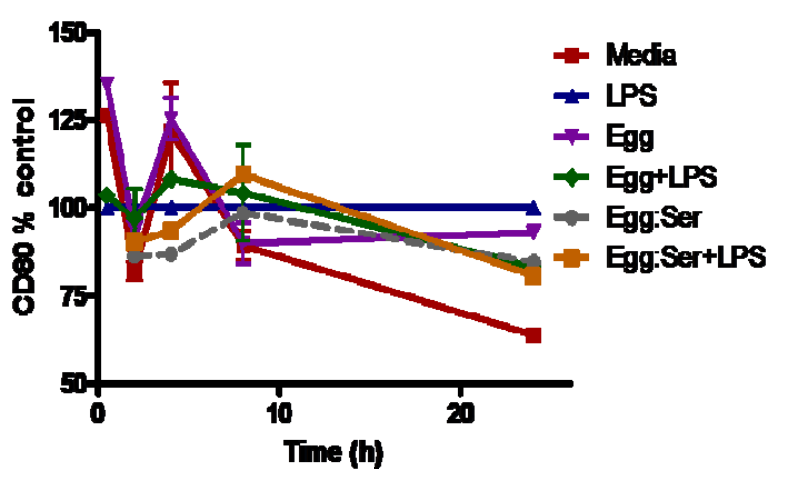

Figure A4 Schistosome egg and egg complex do not induce significant alterations in macrophage surface markers. Primary BMM $\varnothing\left(10^{6} \mathrm{cells} / \mathrm{ml}\right)$ derived from C57BL/6 mice were primed with 20 $\mathrm{U} / \mathrm{ml}$ IFN $\gamma$ overnight followed by stimulation with Media, LPS $(200 \mathrm{ng} / \mathrm{ml})$, Schistosome egg (1000/well), Egg+LPS, opsonised Egg (Egg:ser), or opsonised egg + LPS (Egg:Ser+LPS). Cells were also exposed to Egg of Egg:ser in the absence of LPS and incubated at $37^{\circ} \mathrm{C}$ in $5 \% \mathrm{CO}_{2}$. After $0.5,2,4,8$ and $24 \mathrm{~h}$ culture CD40 (a), CD80 (b) and PDL-1 (c) were assessed via flow cytometry. Data represents the mean +SEM of two experiments, except for the $24 \mathrm{~h}$ time point which represents one experiment. Data is presented as \% of control compared to cultures with LPS. 


\section{Graph illustrating the similar effect 1000 and 2000 schistosome eggs per well have on macrophage activation}

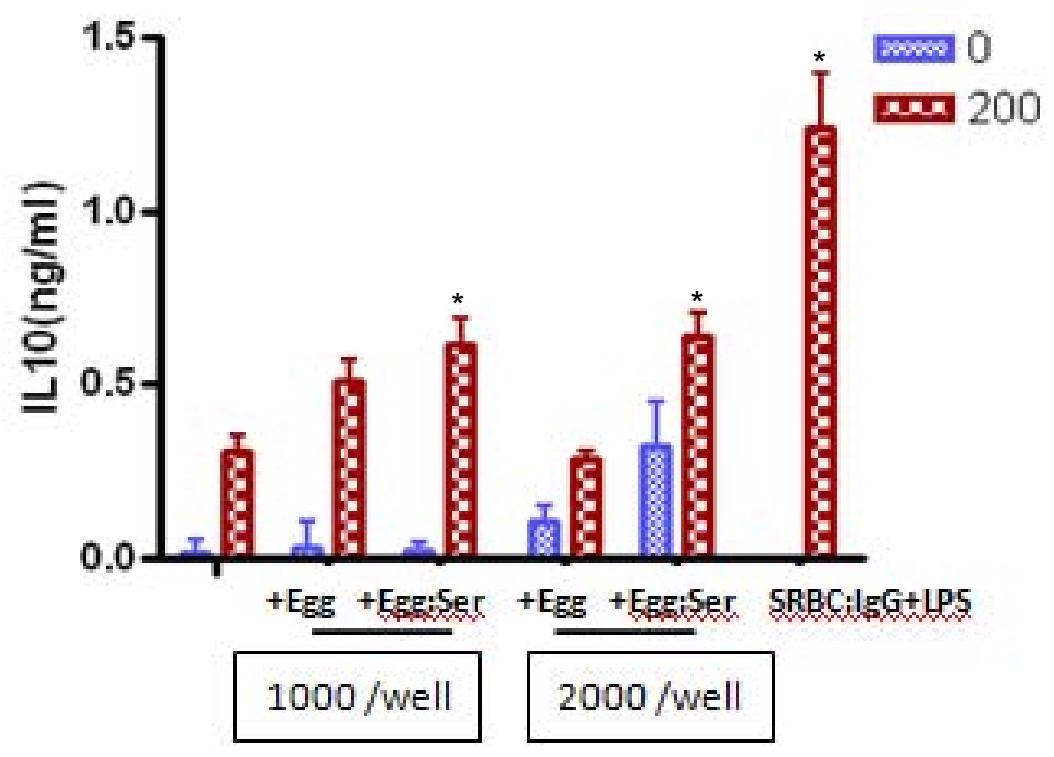

Figure A5 Schistosome eggs at either 1000/well or 2000/well elicit similar responses Primary BMM $\varnothing\left(10^{6} \mathrm{cells} / \mathrm{ml}\right)$ derived from C57BL/6 mice were primed with $20 \mathrm{U} / \mathrm{ml}$ IFN $\gamma$ overnight followed by stimulation with Media, LPS $(200 \mathrm{ng} / \mathrm{ml})$, Schistosome egg either at $1000 /$ well or $2000 /$ well in the absence of presence of LPS, or Egg complexed to serum (Egg:Ser) in the presence or absence of LPS. After $8 \mathrm{~h}$ culture IL-12 was assessed via ELISA. ${ }^{*} \mathrm{P}<0.05$. One way ANOVA followed by Bonferroni post test; treatments vs LPS. Graphs represent the mean + SEM from duplicate wells. 
Optimisation of IFN $\gamma$ concentration for priming macrophages prior to stimulation- identifying which concentration provides the greatest inflammatory response

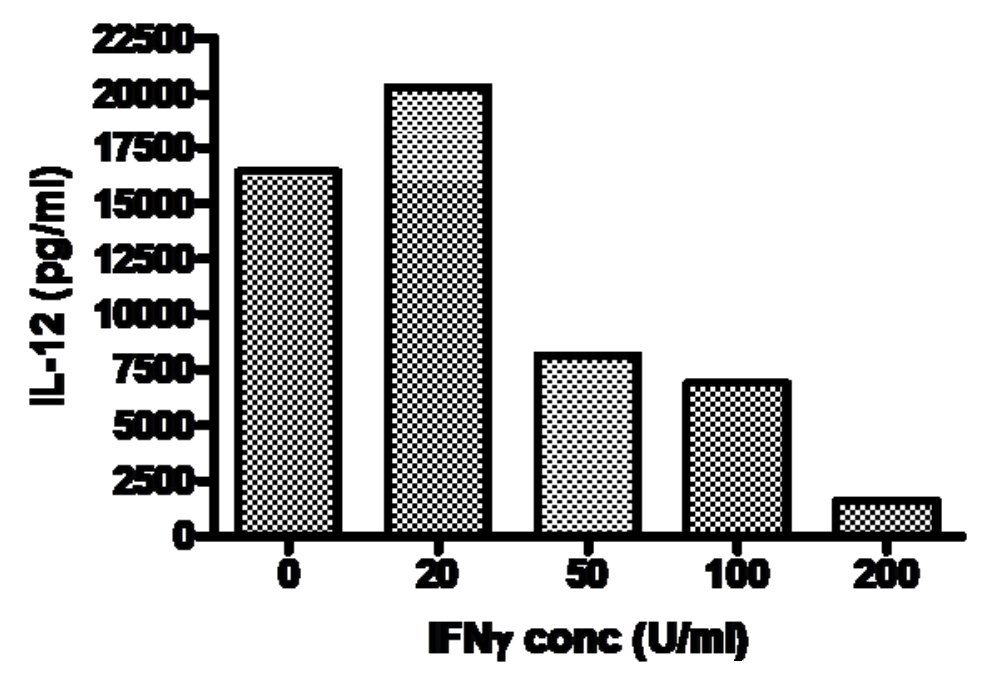

Figure A6 Priming macrophages with $20 \mathrm{U} / \mathrm{ml}$ IFN $\gamma$ generates the most inflammatory conditions for further investigations. Primary BMM $\varnothing\left(10^{6} \mathrm{cells} / \mathrm{ml}\right)$ derived from $\mathrm{C} 57 \mathrm{BL} / 6$ mice were primed with $20,50,100$ or $200 \mathrm{U} / \mathrm{ml} \mathrm{IFN} \gamma$ overnight followed by stimulation with LPS $(200 \mathrm{ng} / \mathrm{ml})$, and incubated at $37^{\circ} \mathrm{C}$ in $5 \% \mathrm{CO}_{2}$. After $8 \mathrm{~h}$ culture, IL-12 was assessed via ELISA. 


\section{Appendix B-Recipes}

Isolation buffer (For CD4 T cell isolation)

2\% FCS (ICP biologicals, Auckland)

2 mM EDTA (Merk)

$1 \mathrm{x}$ dPBS (Invitrogen, Auckland,NZ)

Phosphate buffered saline (PBS) (10x)

For $2 \mathrm{~L}$ of $10 \mathrm{X}$ PBS:

$170 \mathrm{~g} \mathrm{NaCl}$ (Merck Ltd, Palmerston North, New Zealand)

$62.32 \mathrm{~g} \mathrm{Na}_{2} \mathrm{HPO}_{4} .12 \mathrm{H}_{2} \mathrm{O}$ (Merck)

$4.04 \mathrm{~g} \mathrm{NaH}_{2} \mathrm{pPO}_{4} .2 \mathrm{H}_{2} \mathrm{O}$ (Merck)

$2 \mathrm{~L} \mathrm{ddH} \mathrm{H}_{2} \mathrm{O}$

Facs Buffer (For flow cytometry)

$0.1 \%$ sodium azide

97.9\% PBS

2\% FCS (ICP biologicals, Auckland)

\section{Complete T cell medium (CTCM)}

$10 \%$ FCS

$1 \%$ Hepes, Penstrep, Non-essential amino acids, L-Glutamine (Invitrogen)

$0.1 \% \beta$-Mercaptoethanol (Invitrogen)

85.9\% Dulbecco's modified eagle medium (dMEM) ( Invitrogen)

Wash buffer

96\% Dulbecco's modified eagle medium (dMEM) ( Invitrogen)

$3 \%$ Hepes

$1 \%$ Penstrep 
Flow cytometry antibodies

\begin{tabular}{|l|l|}
\hline Antibody & Company \\
\hline Anti- CD40- PE (3/23) & (BD Bioscience) \\
\hline Anti-CD80- FITC (16-10A1) & (BD Biosciences) \\
\hline Anti-IgG2a -PE (eBR2a) & (BD Bioscience) \\
\hline Anti- I-A - PE (AF6-120.1) & (BD Biosciences) \\
\hline Anti-F4/80 bio (C1:A3-1) & (Serotec, Oxford, UK) \\
\hline Anti- CD124-PE (mIL4-M1) & (BD Biosciences) \\
\hline Anti-PDL-1- PE (M1H5) & (eBioscience, CA, USA) \\
\hline Anti-PDL-2- PE (TY25) & (eBiosciences) \\
\hline Anti-mouse-CD16/32 (2.4G2) & (BD Biosciences) \\
\hline Anti-IgG2a- FITC (R35-95) & (BD Biosciences) \\
\hline Anti-CD25-FITC (3C7) & (BD Biosciences) \\
\hline Anti-CD4-Cyc (RM4-5) & (BD Biosciences) \\
\hline
\end{tabular}

Tris buffered saline 10X (TBS) $(500 \mathrm{ml})$

(Tris $200 \mathrm{mM}$, Nacl 1.36 M, pH 7.6)

$2.1 \mathrm{~g}$ Tris, $40 \mathrm{~g} \mathrm{NaCl}, 12 \mathrm{ml} \mathrm{HCl}$ to give $\mathrm{pH} 7.6, \mathrm{ddH}_{2} \mathrm{O}$ up to $500 \mathrm{ml}$

Tween Tris buffered saline (TTBS)

$995 \mathrm{ml}$ TBS (1X), $5 \mathrm{ml}$ Tween-20

Tris glycine buffer X5

(Tris $125 \mathrm{mM}$, Glycine $950 \mathrm{mM}$ )

Glycine $72.1 \mathrm{~g}$, Tris $15.1 \mathrm{~g}, \mathrm{ddH}_{2} \mathrm{O}$ up to $1 \mathrm{~L}$ 


\section{Working transfer buffer}

(Tris 25 mM, Glycine 192 mM , methanol 10\% (v/v))

$600 \mathrm{ml} \mathrm{ddH} \mathrm{H}_{2} \mathrm{O}, 200 \mathrm{ml}$ Tris Glycine buffer $5 \mathrm{X}, 200 \mathrm{ml}$ methanol

\section{Running buffer SDS}

(Tris 25 mM, Glycine 192 mM, SDS 0.1\%)

$1.0 \mathrm{~g}$ SDS, $200 \mathrm{ml}$ Tris Glycine buffer $5 \mathrm{X}, \mathrm{ddH}_{2} \mathrm{O}$ up to $1 \mathrm{~L}$

\section{RIPA Buffer}

Tris-HCl: $1.2110, \mathrm{NaCl} 8.766$,EDTA: 0.3722 ,TritonX-100: $10 \mathrm{ml}$,SDS: 1.0 ,sodium deoxycholate: 10.0 (in g/L) .

\section{Western Blott Gels:}

$10 \%$ separating gel:

$\mathrm{ddH} 20-8 \mathrm{ml}$

$1.5 \mathrm{M}$ tris $8.8-5 \mathrm{ml}$

10\% SDS - $200 \mu \mathrm{l}$ (USB, Cleveland,Ohio)

Acrylamide - $6.66 \mathrm{ml}$ ( Biorad)

$10 \%$ ammonium persulfate (APS) $-100 \mu \mathrm{l}$

TEMED - $10 \mu \mathrm{l}$ (Amersham, England)

$4 \%$ stacking gel:

$\mathrm{ddH} 20-6.1 \mathrm{ml}$

$1.5 \mathrm{M}$ tris $\mathrm{pH} 6.8-2.5 \mathrm{ml}$

$10 \%$ SDS - $100 \mu \mathrm{l}$

acrylamide $-1.33 \mathrm{ml}$

$10 \%$ APS $-50 \mu \mathrm{l}$

TEMED - $10 \mu \mathrm{l}$ 


\section{References}

Abbas, AK, Murphy, KM, Sher, A (1996) Functional diversity of helper T lymphocytes. Nature 383(6603): 787-793.

Abu-Amer, Y, Bar-Shavit, Z (1994) Regulation of TNF-alpha release from bone marrow-derived macrophages by vitamin D. J Cell Biochem 55(4): 435-444.

Aderem, A, Underhill, DM (1999) Mechanisms of phagocytosis in macrophages. Annu Rev Immunol 17: 593-623.

Akira, S, Hirano, T, Taga, T, Kishimoto, T (1990) Biology of multifunctional cytokines: IL 6 and related molecules (IL 1 and TNF). FASEB J 4(11): 2860-2867.

Akira, S, Takeda, K (2004) Toll-like receptor signalling. Nat Rev Immunol 4(7): 499-511.

Albina, JE, Mills, CD, Henry, WL, Jr., Caldwell, MD (1990) Temporal expression of different pathways of 1-arginine metabolism in healing wounds. J Immunol 144(10): 3877-3880.

Alderson, MR, Armitage, RJ, Tough, TW, Strockbine, L, Fanslow, WC, Spriggs, MK (1993) CD40 expression by human monocytes: regulation by cytokines and activation of monocytes by the ligand for CD40. J Exp Med 178(2): 669-674.

Alderton, WK, Cooper, CE, Knowles, RG (2001) Nitric oxide synthases: structure, function and inhibition. Biochem J 357(Pt 3): 593-615.

Alegre, ML, Frauwirth, KA, Thompson, CB (2001) T-cell regulation by CD28 and CTLA-4. Nat Rev Immunol 1(3): 220-228.

Anderson, CF, Gerber, JS, Mosser, DM (2002a) Modulating macrophage function with IgG immune complexes. J Endotoxin Res 8(6): 477-481.

Anderson, CF, Lucas, M, Gutierrez-Kobeh, L, Field, AE, Mosser, DM (2004) T cell biasing by activated dendritic cells. J Immunol 173(2): 955-961.

Anderson, CF, Mosser, DM (2002b) Cutting edge: biasing immune responses by directing antigen to macrophage Fc gamma receptors. J Immunol 168(8): 3697-3701.

Armitage, RJ, Tough, TW, Macduff, BM, Fanslow, WC, Spriggs, MK, Ramsdell, F, Alderson, MR (1993) CD40 ligand is a T cell growth factor. Eur J Immunol 23(9): 2326-2331.

Attwood, JT, Munn, DH (1999) Macrophage suppression of T cell activation: a potential mechanism of peripheral tolerance. Int Rev Immunol 18(5-6): 515-525.

Banchereau, J, Bazan, F, Blanchard, D, Briere, F, Galizzi, JP, van Kooten, C, Liu, YJ, Rousset, F, Saeland, S (1994) The CD40 antigen and its ligand. Annu Rev Immunol 12: 881-922.

Baranzini, SE, Elfstrom, C, Chang, S-Y, Butunoi, C, Murray, R, Higuchi, R, Oksenberg, JR (2000) Transcriptional Analysis of Multiple Sclerosis Brain Lesions Reveals a Complex Pattern of Cytokine Expression. J Immunol 165(11): 6576-6582. 
Barker, RN, Erwig, LP, Hill, KS, Devine, A, Pearce, WP, Rees, AJ (2002) Antigen presentation by macrophages is enhanced by the uptake of necrotic, but not apoptotic, cells. Clin Exp Immunol 127(2): 220-225.

Barry, M, Bleackley, RC (2002) Cytotoxic T lymphocytes: all roads lead to death. Nat Rev Immunol 2(6): 401-409.

Becher, B, Blain, M, Antel, JP (2000) CD40 engagement stimulates IL-12 p70 production by human microglial cells: basis for Th1 polarization in the CNS. J Neuroimmunol 102(1): 44-50.

Becher, B, Durell, BG, Noelle, RJ (2002) Experimental autoimmune encephalitis and inflammation in the absence of interleukin-12. J Clin Invest 110(4): 493-497.

Begolka, WS, Vanderlugt, CL, Rahbe, SM, Miller, SD (1998) Differential Expression of Inflammatory Cytokines Parallels Progression of Central Nervous System Pathology in Two Clinically Distinct Models of Multiple Sclerosis. J Immunol 161(8): 4437-4446.

Bettelli, E, Carrier, Y, Gao, W, Korn, T, Strom, TB, Oukka, M, Weiner, HL, Kuchroo, VK (2006) Reciprocal developmental pathways for the generation of pathogenic effector TH17 and regulatory T cells. Nature 441(7090): 235-238.

Billich, A, Bornancin, F, DÃ@vay, P, Mechtcheriakova, D, Urtz, N, Baumruker, T (2003) Phosphorylation of the Immunomodulatory Drug FTY720 by Sphingosine Kinases. Journal of Biological Chemistry 278(48): 47408-47415.

Bird, JJ, Brown, DR, Mullen, AC, Moskowitz, NH, Mahowald, MA, Sider, JR, Gajewski, TF, Wang, CR, Reiner, SL (1998) Helper T cell differentiation is controlled by the cell cycle. Immunity 9(2): 229237.

Bjerke, T, Gaustadnes, M, Nielsen, S, Nielsen, LP, Schiotz, PO, Rudiger, N, Reimert, CM, Dahl, R, Christensen, I, Poulsen, LK (1996) Human blood eosinophils produce and secrete interleukin 4. Respir Med 90(5): 271-277.

Bogdan, C, Vodovotz, Y, Nathan, C (1991) Macrophage deactivation by interleukin 10. J Exp Med 174(6): 1549-1555.

Boros, D. L. \& Warren, K. S. (1970) Delayed hypersensitivity-type granuloma formation and dermal reaction induced and elicited by a soluble factor isolated from Schistosoma mansoni eggs. J Exp Med, 132, 488-507.

Bowdish, DM, Loffredo, MS, Mukhopadhyay, S, Mantovani, A, Gordon, S (2007) Macrophage receptors implicated in the "adaptive" form of innate immunity. Microbes Infect 9(14-15): 16801687.

Brem-Exner, BG, Sattler, C, Hutchinson, JA, Koehl, GE, Kronenberg, K, Farkas, S, Inoue, S, Blank, C, Knechtle, SJ, Schlitt, HJ, Fandrich, F, Geissler, EK (2008) Macrophages driven to a novel state of activation have anti-inflammatory properties in mice. J Immunol 180(1): 335-349.

Brunet, LR, Beall, M, Dunne, DW, Pearce, EJ (1999) Nitric oxide and the Th2 response combine to prevent severe hepatic damage during Schistosoma mansoni infection. J Immunol 163(9): 49764984. 
Butterworth, AE, Curry, AJ, Dunne, DW, Fulford, AJ, Kimani, G, Kariuki, HC, Klumpp, R, Koech, D, Mbugua, G, Ouma, JH, et al. (1994) Immunity and morbidity in human schistosomiasis mansoni. Trop Geogr Med 46(4 Spec No): 197-208.

Buttner, C, Skupin, A, Reimann, T, Rieber, EP, Unteregger, G, Geyer, P, Frank, KH (1997) Local production of interleukin-4 during radiation-induced pneumonitis and pulmonary fibrosis in rats: macrophages as a prominent source of interleukin-4. Am J Respir Cell Mol Biol 17(3): 315-325.

Campbell, KA, Ovendale, PJ, Kennedy, MK, Fanslow, WC, Reed, SG, Maliszewski, CR (1996) CD40 ligand is required for protective cell-mediated immunity to Leishmania major. Immunity 4(3): 283289.

Caux, C, Burdin, N, Galibert, L, Hermann, P, Renard, N, Servet-Delprat, C, Banchereau, J (1994a) Functional CD40 on B lymphocytes and dendritic cells. Res Immunol 145(3): 235-239; discussion 244-239.

Caux, C, Massacrier, C, Vanbervliet, B, Dubois, B, Van Kooten, C, Durand, I, Banchereau, J (1994b) Activation of human dendritic cells through CD40 cross-linking. J Exp Med 180(4): 1263-1272.

Chatelain, R, Varkila, K, Coffman, RL (1992) IL-4 induces a Th2 response in Leishmania majorinfected mice. J Immunol 148(4): 1182-1187.

Chitsulo, L, Engels, D, Montresor, A, Savioli, L (2000) The global status of schistosomiasis and its control. Acta Trop 77(1): 41-51.

Chow, JC, Young, DW, Golenbock, DT, Christ, WJ, Gusovsky, F (1999) Toll-like Receptor-4 Mediates Lipopolysaccharide-induced Signal Transduction. Journal of Biological Chemistry 274(16): 1068910692.

Ciesielski, CJ, Andreakos, E, Foxwell, BM, Feldmann, M (2002) TNFalpha-induced macrophage chemokine secretion is more dependent on NF-kappaB expression than lipopolysaccharidesinduced macrophage chemokine secretion. Eur J Immunol 32(7): 2037-2045.

Clarke, SR (2000) The critical role of CD40/CD40L in the CD4-dependent generation of CD8+ T cell immunity. J Leukoc Biol 67(5): 607-614.

Coffman, RL, Lebman, DA, Rothman, P (1993) Mechanism and regulation of immunoglobulin isotype switching. Adv Immunol 54: 229-270.

Cook, SD (1996) REVIEW : The Epidemiology of Multiple Sclerosis: Clues to the Etiology of a Mysterious Disease. The Neuroscientist 2(3): 172-180.

Cooke, A, Tonks, P, Jones, FM, O'Shea, H, Hutchings, P, Fulford, AJ, Dunne, DW (1999) Infection with Schistosoma mansoni prevents insulin dependent diabetes mellitus in non-obese diabetic mice. Parasite Immunol 21(4): 169-176.

Corraliza, IM, Soler, G, Eichmann, K, Modolell, M (1995) Arginase induction by suppressors of nitric oxide synthesis (IL-4, IL-10 and PGE2) in murine bone-marrow-derived macrophages. Biochem Biophys Res Commun 206(2): 667-673.

Cote-Sierra, J, Foucras, G, Guo, L, Chiodetti, L, Young, HA, Hu-Li, J, Zhu, J, Paul, WE (2004) Interleukin 2 plays a central role in Th2 differentiation. Proc Natl Acad Sci U S A 101(11): 38803885 . 
Creagh, EM, O'Neill, LA (2006) TLRs, NLRs and RLRs: a trinity of pathogen sensors that co-operate in innate immunity. Trends Immunol 27(8): 352-357.

Crume, KP, Miller, JH, La Flamme, AC (2007) Peloruside A, an antimitotic agent, specifically decreases tumor necrosis factor-alpha production by lipopolysaccharide-stimulated murine macrophages. Exp Biol Med (Maywood) 232(5): 607-613.

Cua, D, Hinton, D, Stohlman, S (1995) Self-antigen-induced Th2 responses in experimental allergic encephalomyelitis (EAE)-resistant mice. Th2-mediated suppression of autoimmune disease. J Immunol 155(8): 4052-4059.

Cua, DJ, Sherlock, J, Chen, Y, Murphy, CA, Joyce, B, Seymour, B, Lucian, L, To, W, Kwan, S, Churakova, T, Zurawski, S, Wiekowski, M, Lira, SA, Gorman, D, Kastelein, RA, Sedgwick, JD (2003) Interleukin-23 rather than interleukin-12 is the critical cytokine for autoimmune inflammation of the brain. Nature 421(6924): 744-748.

Curtsinger, JM, Schmidt, CS, Mondino, A, Lins, DC, Kedl, RM, Jenkins, MK, Mescher, MF (1999) Inflammatory cytokines provide a third signal for activation of naive CD4+ and CD8+ T cells. $J$ Immunol 162(6): 3256-3262.

Dalton P. J. (1997) A method for the isolation of schistosome eggs and miracidia free of contaminating host tissues. Parasitology, 115 ( Pt 1), 29-32.

D'Andrea, A, Aste-Amezaga, M, Valiante, NM, Ma, X, Kubin, M, Trinchieri, G (1993) Interleukin 10 (IL-10) inhibits human lymphocyte interferon gamma-production by suppressing natural killer cell stimulatory factor/IL-12 synthesis in accessory cells. J Exp Med 178(3): 1041-1048.

de Jesus, AR, Silva, A, Santana, LB, Magalhaes, A, de Jesus, AA, de Almeida, RP, Rego, MA, Burattini, MN, Pearce, EJ, Carvalho, EM (2002) Clinical and immunologic evaluation of 31 patients with acute schistosomiasis mansoni. J Infect Dis 185(1): 98-105.

de Waal Malefyt, R, Abrams, J, Bennett, B, Figdor, CG, de Vries, JE (1991) Interleukin 10(IL-10) inhibits cytokine synthesis by human monocytes: an autoregulatory role of IL-10 produced by monocytes. The Journal of Experimental Medicine 174(5): 1209-1220.

DeKruyff, RH, Gieni, RS, Umetsu, DT (1997) Antigen-driven but not lipopolysaccharide-driven IL-12 production in macrophages requires triggering of CD40. J Immunol 158(1): 359-366.

Dinapoli, MR, Calderon, CL, Lopez, DM (1996) The altered tumoricidal capacity of macrophages isolated from tumor-bearing mice is related to reduce expression of the inducible nitric oxide synthase gene. J Exp Med 183(4): 1323-1329.

Dunn, DL, Barke, RA, Knight, NB, Humphrey, EW, Simmons, RL (1985) Role of resident macrophages, peripheral neutrophils, and translymphatic absorption in bacterial clearance from the peritoneal cavity. Infect Immun 49(2): 257-264.

Dunne, DW, Cooke, A (2005) A worm's eye view of the immune system: consequences for evolution of human autoimmune disease. Nat Rev Immunol 5(5): 420-426. 
Dyment, DA, Herrera, BM, Cader, MZ, Willer, CJ, Lincoln, MR, Sadovnick, AD, Risch, N, Ebers, GC (2005) Complex interactions among MHC haplotypes in multiple sclerosis: susceptibility and resistance. Hum. Mol. Genet. 14(14): 2019-2026.

Edwards, JP, Zhang, X, Frauwirth, KA, Mosser, DM (2006) Biochemical and functional characterization of three activated macrophage populations. Journal of Leukocyte Biology 80(6): 1298-1307.

Elenkov, IJ (2004) Glucocorticoids and the Th1/Th2 balance. Ann N Y Acad Sci 1024: 138-146.

Fiorentino, DF, Zlotnik, A, Vieira, P, Mosmann, TR, Howard, M, Moore, KW, O'Garra, A (1991) IL-10 acts on the antigen-presenting cell to inhibit cytokine production by Th1 cells. J Immunol 146(10): 3444-3451.

Fleming, J, Fabry, Z (2007) The hygiene hypothesis and multiple sclerosis. Ann Neurol 61(2): 85-89.

Florido, M, Goncalves, AS, Gomes, MS, Appelberg, R (2004) CD40 is required for the optimal induction of protective immunity to Mycobacterium avium. Immunology 111(3): 323-327.

Fontenot, JD, Gavin, MA, Rudensky, AY (2003) Foxp3 programs the development and function of CD4+CD25+ regulatory T cells. Nat Immunol 4(4): 330-336.

Fontenot, JD, Rasmussen, JP, Williams, LM, Dooley, JL, Farr, AG, Rudensky, AY (2005) Regulatory T cell lineage specification by the forkhead transcription factor foxp3. Immunity 22(3): 329-341.

Freeman, GJ, Long, AJ, Iwai, Y, Bourque, K, Chernova, T, Nishimura, H, Fitz, L, Malenkovich, N, Okazaki, T, Byrne, MC, Horton, HF, Fouser, L, Carter, L, Ling, V, Bowman, MR, Carreno, BM, Collins, M, Wood, CR, Honjo, T (2000) Engagement of the PD-1 immunoinhibitory receptor by a novel B7 family member leads to negative regulation of lymphocyte activation. J Exp Med 192(7): 10271034.

Gerber, JS, Mosser, DM (2001) Reversing lipopolysaccharide toxicity by ligating the macrophage Fc gamma receptors. J Immunol 166(11): 6861-6868.

Germann, T, Gately, MK, Schoenhaut, DS, Lohoff, M, Mattner, F, Fischer, S, Jin, SC, Schmitt, E, Rude, E (1993) Interleukin-12/T cell stimulating factor, a cytokine with multiple effects on Thelper type 1 (Th1) but not on Th2 cells. Eur J Immunol 23(8): 1762-1770.

Girvin, AM, Dal Canto, MC, Rhee, L, Salomon, B, Sharpe, A, Bluestone, JA, Miller, SD (2000) A Critical Role for B7/CD28 Costimulation in Experimental Autoimmune Encephalomyelitis: $A$ Comparative Study Using Costimulatory Molecule-Deficient Mice and Monoclonal Antibody Blockade. J Immunol 164(1): 136-143.

Gordon, S (2007) The macrophage: past, present and future. Eur J Immunol 37 Suppl 1: S9-17.

Gordon, S, Taylor, PR (2005) Monocyte and macrophage heterogeneity. Nat Rev Immunol 5(12): 953-964.

Grazia Cappiello, M, Sutterwala, FS, Trinchieri, G, Mosser, DM, Ma, X (2001) Suppression of IL-12 Transcription in Macrophages Following Fc\{\{gamma\}\} Receptor Ligation. J Immunol 166(7): 44984506. 
Grewal, IS, Flavell, RA (1998) CD40 and CD154 in cell-mediated immunity. Annu Rev Immunol 16: 111-135.

Grewal, IS, Foellmer, HG, Grewal, KD, Xu, J, Hardardottir, F, Baron, JL, Janeway, CA, Jr., Flavell, RA (1996) Requirement for CD40 ligand in costimulation induction, $T$ cell activation, and experimental allergic encephalomyelitis. Science 273(5283): 1864-1867.

Groux, H, O'Garra, A, Bigler, M, Rouleau, M, Antonenko, S, de Vries, JE, Roncarolo, MG (1997) A CD4+ T-cell subset inhibits antigen-specific T-cell responses and prevents colitis. Nature 389(6652): 737-742.

Grzych, JM, Pearce, E, Cheever, A, Caulada, ZA, Caspar, P, Heiny, S, Lewis, F, Sher, A (1991) Egg deposition is the major stimulus for the production of Th2 cytokines in murine schistosomiasis mansoni. J Immunol 146(4): 1322-1327.

Hancock, WW, Sayegh, MH, Zheng, XG, Peach, R, Linsley, PS, Turka, LA (1996) Costimulatory function and expression of CD40 ligand, CD80, and CD86 in vascularized murine cardiac allograft rejection. Proc Natl Acad Sci U S A 93(24): 13967-13972.

Heinzel, FP, Schoenhaut, DS, Rerko, RM, Rosser, LE, Gately, MK (1993) Recombinant interleukin 12 cures mice infected with Leishmania major. J Exp Med 177(5): 1505-1509.

Held, TK, Weihua, X, Yuan, L, Kalvakolanu, DV, Cross, AS (1999) Gamma interferon augments macrophage activation by lipopolysaccharide by two distinct mechanisms, at the signal transduction level and via an autocrine mechanism involving tumor necrosis factor alpha and interleukin-1. Infect Immun 67(1): 206-212.

Herbert, DR, Holscher, C, Mohrs, M, Arendse, B, Schwegmann, A, Radwanska, M, Leeto, M, Kirsch, R, Hall, P, Mossmann, H, Claussen, B, Forster, I, Brombacher, F (2004) Alternative macrophage activation is essential for survival during schistosomiasis and downmodulates Thelper 1 responses and immunopathology. Immunity 20(5): 623-635.

Herbert, DR, Orekov, T, Perkins, C, Rothenberg, ME, Finkelman, FD (2008) IL-4R alpha expression by bone marrow-derived cells is necessary and sufficient for host protection against acute schistosomiasis. J Immunol 180(7): 4948-4955.

Hesse, M, Modolell, M, La Flamme, AC, Schito, M, Fuentes, JM, Cheever, AW, Pearce, EJ, Wynn, TA (2001) Differential regulation of nitric oxide synthase- 2 and arginase-1 by type 1/type 2 cytokines in vivo: granulomatous pathology is shaped by the pattern of L-arginine metabolism. $J$ Immunol 167(11): 6533-6544.

Hesse, M, Piccirillo, CA, Belkaid, Y, Prufer, J, Mentink-Kane, M, Leusink, M, Cheever, AW, Shevach, EM, Wynn, TA (2004) The Pathogenesis of Schistosomiasis Is Controlled by Cooperating IL-10Producing Innate Effector and Regulatory T Cells. J Immunol 172(5): 3157-3166.

Hill, A, Chapel, H (1993) X-linked immunodeficiency. The fruits of cooperation. Nature 361(6412): 494.

Hoffmann, KF, Cheever, AW, Wynn, TA (2000) IL-10 and the Dangers of Immune Polarization: Excessive Type 1 and Type 2 Cytokine Responses Induce Distinct Forms of Lethal Immunopathology in Murine Schistosomiasis. J Immunol 164(12): 6406-6416. 
Hoshino, K, Takeuchi, O, Kawai, T, Sanjo, H, Ogawa, T, Takeda, Y, Takeda, K, Akira, S (1999) Cutting Edge: Toll-Like Receptor 4 (TLR4)-Deficient Mice Are Hyporesponsive to Lipopolysaccharide: Evidence for TLR4 as the Lps Gene Product. J Immunol 162(7): 3749-3752.

Howard, LM, Miga, AJ, Vanderlugt, CL, Dal Canto, MC, Laman, JD, Noelle, RJ, Miller, SD (1999) Mechanisms of immunotherapeutic intervention by anti-CD40L (CD154) antibody in an animal model of multiple sclerosis. J Clin Invest 103(2): 281-290.

Howard, M, Muchamuel, T, Andrade, S, Menon, S (1993) Interleukin 10 protects mice from lethal endotoxemia. J Exp Med 177(4): 1205-1208.

Hu, W, Brindley, PJ, McManus, DP, Feng, Z, Han, ZG (2004) Schistosome transcriptomes: new insights into the parasite and schistosomiasis. Trends Mol Med 10(5): 217-225.

Huang, D, Wang, J, Kivisakk, P, Rollins, BJ, Ransohoff, RM (2001a) Absence of Monocyte Chemoattractant Protein 1 in Mice Leads to Decreased Local Macrophage Recruitment and Antigen-Specific $T$ Helper Cell Type 1 Immune Response in Experimental Autoimmune Encephalomyelitis. The Journal of Experimental Medicine 193(6): 713-726.

Huang, DR, Wang, J, Kivisakk, P, Rollins, BJ, Ransohoff, RM (2001b) Absence of monocyte chemoattractant protein 1 in mice leads to decreased local macrophage recruitment and antigenspecific $T$ helper cell type 1 immune response in experimental autoimmune encephalomyelitis. $J$ Exp Med 193(6): 713-726.

Jager, A, Dardalhon, V, Sobel, RA, Bettelli, E, Kuchroo, VK (2009) Th1, Th17, and Th9 effector cells induce experimental autoimmune encephalomyelitis with different pathological phenotypes. $J$ Immunol 183(11): 7169-7177.

Jakubzick, C, Choi, ES, Kunkel, SL, Joshi, BH, Puri, RK, Hogaboam, CM (2003) Impact of interleukin13 responsiveness on the synthetic and proliferative properties of Th1- and Th2-type pulmonary granuloma fibroblasts. Am J Pathol 162(5): 1475-1486.

Janeway, CA, Jr., Medzhitov, R (2002) Innate immune recognition. Annu Rev Immunol 20: 197-216.

Jang, S, Uematsu, S, Akira, S, Salgame, P (2004) IL-6 and IL-10 induction from dendritic cells in response to Mycobacterium tuberculosis is predominantly dependent on TLR2-mediated recognition. J Immunol 173(5): 3392-3397.

Janssens, S, Beyaert, R (2003) Role of Toll-like receptors in pathogen recognition. Clin Microbiol Rev 16(4): 637-646.

Jelley-Gibbs, DM, Dibble, JP, Filipson, S, Haynes, L, Kemp, RA, Swain, SL (2005) Repeated stimulation of CD4 effector T cells can limit their protective function. The Journal of Experimental Medicine 201(7): 1101-1112.

Jelley-Gibbs, DM, Lepak, NM, Yen, M, Swain, SL (2000) Two distinct stages in the transition from naive CD4 T cells to effectors, early antigen-dependent and late cytokine-driven expansion and differentiation. J Immunol 165(9): 5017-5026.

Kane, CM, Jung, E, Pearce, EJ (2008) Schistosoma mansoni egg antigen-mediated modulation of Toll-like receptor (TLR)-induced activation occurs independently of TLR2, TLR4, and MyD88. Infect Immun 76(12): 5754-5759. 
Kaplan, MH, Schindler, U, Smiley, ST, Grusby, MJ (1996) Stat6 is required for mediating responses to IL-4 and for development of Th2 cells. Immunity 4(3): 313-319.

Karandikar, NJ, Vanderlugt, CL, Eagar, T, Tan, L, Bluestone, JA, Miller, SD (1998) Tissue-specific upregulation of B7-1 expression and function during the course of murine relapsing experimental autoimmune encephalomyelitis. J Immunol 161(1): 192-199.

Keir, ME, Liang, SC, Guleria, I, Latchman, YE, Qipo, A, Albacker, LA, Koulmanda, M, Freeman, GJ, Sayegh, MH, Sharpe, AH (2006) Tissue expression of PD-L1 mediates peripheral T cell tolerance. J Exp Med 203(4): 883-895.

Kelsall, BL, Stuber, E, Neurath, M, Strober, W (1996) Interleukin-12 production by dendritic cells. The role of CD40-CD40L interactions in Th1 T-cell responses. Ann N Y Acad Sci 795: 116-126.

Kennedy, M, Torrance, D, Picha, K, Mohler, K (1992) Analysis of cytokine mRNA expression in the central nervous system of mice with experimental autoimmune encephalomyelitis reveals that IL10 mRNA expression correlates with recovery. J Immunol 149(7): 2496-2505.

Kihara, A, Anada, Y, Igarashi, Y (2006) Mouse sphingosine kinase isoforms SPHK1a and SPHK1b differ in enzymatic traits including stability, localization, modification, and oligomerization. J Biol Chem 281(7): 4532-4539.

Kuipers, H, Muskens, F, Willart, M, Hijdra, D, van Assema, FB, Coyle, AJ, Hoogsteden, HC, Lambrecht, BN (2006) Contribution of the PD-1 ligands/PD-1 signaling pathway to dendritic cellmediated CD4+ T cell activation. Eur J Immunol 36(9): 2472-2482.

Kurata, H, Lee, HJ, O'Garra, A, Arai, N (1999) Ectopic expression of activated Stat6 induces the expression of Th2-specific cytokines and transcription factors in developing Th1 cells. Immunity 11(6): 677-688.

Kurtzke, JF (2000) Multiple sclerosis in time and space--geographic clues to cause. J Neurovirol 6 Suppl 2: S134-140.

La Flamme, AC, Canagasabey, K, Harvie, M, Backstrom, BT (2004) Schistosomiasis protects against multiple sclerosis. Mem Inst Oswaldo Cruz 99(5 Suppl 1): 33-36.

La Flamme, AC, Harvie, M, McNeill, A, Goldsack, L, Tierney, JB, Backstrom, BT (2006) Fcgamma receptor-ligating complexes improve the course of experimental autoimmune encephalomyelitis by enhancing basal Th2 responses. Immunol Cell Biol 84(6): 522-529.

La Flamme, AC, MacDonald, AS, Pearce, EJ (2000) Role of IL-6 in directing the initial immune response to schistosome eggs. J Immunol 164(5): 2419-2426.

La Flamme, AC, Patton, EA, Bauman, B, Pearce, EJ (2001) IL-4 plays a crucial role in regulating oxidative damage in the liver during schistosomiasis. J Immunol 166(3): 1903-1911.

La Flamme, AC, Ruddenklau, K, Backstrom, BT (2003) Schistosomiasis decreases central nervous system inflammation and alters the progression of experimental autoimmune encephalomyelitis. Infect Immun 71(9): 4996-5004.

Lamb, JR, Skidmore, BJ, Green, N, Chiller, JM, Feldmann, M (1983) Induction of tolerance in influenza virus-immune T lymphocyte clones with synthetic peptides of influenza hemagglutinin. $J$ Exp Med 157(5): 1434-1447. 
Lang, R, Patel, D, Morris, JJ, Rutschman, RL, Murray, PJ (2002) Shaping Gene Expression in Activated and Resting Primary Macrophages by IL-10. J Immunol 169(5): 2253-2263.

Langrish, CL, Chen, Y, Blumenschein, WM, Mattson, J, Basham, B, Sedgwick, JD, McClanahan, T, Kastelein, RA, Cua, DJ (2005) IL-23 drives a pathogenic T cell population that induces autoimmune inflammation. J Exp Med 201(2): 233-240.

Latchman, Y, Wood, CR, Chernova, T, Chaudhary, D, Borde, M, Chernova, I, Iwai, Y, Long, AJ, Brown, JA, Nunes, R, Greenfield, EA, Bourque, K, Boussiotis, VA, Carter, LL, Carreno, BM, Malenkovich, N, Nishimura, H, Okazaki, T, Honjo, T, Sharpe, AH, Freeman, GJ (2001) PD-L2 is a second ligand for PD-1 and inhibits T cell activation. Nat Immunol 2(3): 261-268.

Le Gros, G, Ben-Sasson, SZ, Seder, R, Finkelman, FD, Paul, WE (1990) Generation of interleukin 4 (IL-4)-producing cells in vivo and in vitro: IL-2 and IL-4 are required for in vitro generation of IL-4producing cells. The Journal of Experimental Medicine 172(3): 921-929.

Lechler, R, Chai, JG, Marelli-Berg, F, Lombardi, G (2001) T-cell anergy and peripheral T-cell tolerance. Philos Trans R Soc Lond B Biol Sci 356(1409): 625-637.

Lee, BO, Haynes, L, Eaton, SM, Swain, SL, Randall, TD (2002) The biological outcome of CD40 signaling is dependent on the duration of $C D 40$ ligand expression: reciprocal regulation by interleukin (IL)-4 and IL-12. J Exp Med 196(5): 693-704.

Lee, I, Wang, L, Wells, AD, Ye, Q, Han, R, Dorf, ME, Kuziel, WA, Rollins, BJ, Chen, L, Hancock, WW (2003) Blocking the monocyte chemoattractant protein-1/CCR2 chemokine pathway induces permanent survival of islet allografts through a programmed death-1 ligand-1-dependent mechanism. J Immunol 171(12): 6929-6935.

Lenschow, DJ, Walunas, TL, Bluestone, JA (1996) CD28/B7 system of T cell costimulation. Annu Rev Immunol 14: 233-258.

Leung, BP, McInnes, IB, Esfandiari, E, Wei, XQ, Liew, FY (2000) Combined effects of IL-12 and IL-18 on the induction of collagen-induced arthritis. J Immunol 164(12): 6495-6502.

Li, J, Li, Y, Yao, JY, Jin, R, Zhu, MZ, Qian, XP, Zhang, J, Fu, YX, Wu, L, Zhang, Y, Chen, WF (2007) Developmental pathway of $C D 4+C D 8$ - medullary thymocytes during mouse ontogeny and its defect in Aire-/- mice. Proc Natl Acad Sci U S A 104(46): 18175-18180.

Li, J, Sutterwala, S, Farrell, JP (1997) Successful therapy of chronic, nonhealing murine cutaneous leishmaniasis with sodium stibogluconate and gamma interferon depends on continued interleukin-12 production. Infect Immun 65(8): 3225-3230.

Liao, W, Schones, DE, Oh, J, Cui, Y, Cui, K, Roh, TY, Zhao, K, Leonard, WJ (2008) Priming for T helper type 2 differentiation by interleukin 2-mediated induction of interleukin 4 receptor alphachain expression. Nat Immunol 9(11): 1288-1296.

Liew, F, Millott, S, Parkinson, C, Palmer, R, Moncada, S (1990) Macrophage killing of Leishmania parasite in vivo is mediated by nitric oxide from L-arginine. J Immunol 144(12): 4794-4797.

Lin, EY, Li, JF, Gnatovskiy, L, Deng, Y, Zhu, L, Grzesik, DA, Qian, H, Xue, XN, Pollard, JW (2006) Macrophages regulate the angiogenic switch in a mouse model of breast cancer. Cancer Res 66(23): 11238-11246. 
Linkhart, TA, Linkhart, SG, MacCharles, DC, Long, DL, Strong, DD (1991) Interleukin-6 messenger RNA expression and interleukin- 6 protein secretion in cells isolated from normal human bone: regulation by interleukin-1. J Bone Miner Res 6(12): 1285-1294.

Linsley, PS, Brady, W, Grosmaire, L, Aruffo, A, Damle, NK, Ledbetter, JA (1991) Binding of the B cell activation antigen $B 7$ to $C D 28$ costimulates $T$ cell proliferation and interleukin 2 mRNA accumulation. J Exp Med 173(3): 721-730.

Loke, P, Allison, JP (2003) PD-L1 and PD-L2 are differentially regulated by Th1 and Th2 cells. Proc Natl Acad Sci U S A 100(9): 5336-5341.

Lucas, M, Zhang, X, Prasanna, V, Mosser, DM (2005) ERK Activation Following Macrophage Fc\{gamma\}R Ligation Leads to Chromatin Modifications at the IL-10 Locus. J Immunol 175(1): 469477.

Lyons, AB, Parish, CR (1994) Determination of lymphocyte division by flow cytometry. J Immunol Methods 171(1): 131-137.

Ma, X (2001) TNF-alpha and IL-12: a balancing act in macrophage functioning. Microbes Infect 3(2): 121-129.

Macaulay, AE, Dekruyff, RH, Goodnow, CC, Umetsu, DT (1997) Antigen-specific B cells preferentially induce CD4+ T cells to produce IL-4. J Immunol 158(9): 4171-4179.

MacDonald, AS, Patton, EA, La Flamme, AC, Araujo, MI, Huxtable, CR, Bauman, B, Pearce, EJ (2002a) Impaired Th2 Development and Increased Mortality During Schistosoma mansoni Infection in the Absence of CD40/CD154 Interaction. J Immunol 168(9): 4643-4649.

MacDonald, AS, Patton, EA, La Flamme, AC, Araujo, MI, Huxtable, CR, Bauman, B, Pearce, EJ (2002b) Impaired Th2 development and increased mortality during Schistosoma mansoni infection in the absence of CD40/CD154 interaction. J Immunol 168(9): 4643-4649.

MacDonald, AS, Straw, AD, Dalton, NM, Pearce, EJ (2002c) Cutting edge: Th2 response induction by dendritic cells: a role for CD40. J Immunol 168(2): 537-540.

MacMicking, J, Xie, QW, Nathan, C (1997) Nitric oxide and macrophage function. Annu Rev Immunol 15: 323-350.

Maggi, E, Parronchi, P, Manetti, R, Simonelli, C, Piccinni, MP, Rugiu, FS, De Carli, M, Ricci, M, Romagnani, S (1992) Reciprocal regulatory effects of IFN-gamma and IL-4 on the in vitro development of human Th1 and Th2 clones. J Immunol 148(7): 2142-2147.

Magram, J, Connaughton, SE, Warrier, RR, Carvajal, DM, Wu, CY, Ferrante, J, Stewart, C, Sarmiento, U, Faherty, DA, Gately, MK (1996) IL-12-deficient mice are defective in IFN gamma production and type 1 cytokine responses. Immunity 4(5): 471-481.

Maimone, D, Guazzi, GC, Annunziata, P (1997) IL-6 detection in multiple sclerosis brain. J Neurol Sci 146(1): 59-65.

Manetti, R, Parronchi, P, Giudizi, MG, Piccinni, MP, Maggi, E, Trinchieri, G, Romagnani, S (1993) Natural killer cell stimulatory factor (interleukin 12 [IL-12]) induces T helper type 1 (Th1)-specific 
immune responses and inhibits the development of IL-4-producing Th cells. J Exp Med 177(4): 1199-1204.

Mantovani, A, Garlanda, C, Locati, M, Rodriguez, TV, Feo, SG, Savino, B, Vecchi, A (2007) Regulatory pathways in inflammation. Autoimmun Rev 7(1): 8-11.

Mantovani, A, Sica, A, Sozzani, S, Allavena, P, Vecchi, A, Locati, M (2004) The chemokine system in diverse forms of macrophage activation and polarization. Trends Immunol 25(12): 677-686.

Mantovani, A, Sozzani, S, Locati, M, Allavena, P, Sica, A (2002) Macrophage polarization: tumorassociated macrophages as a paradigm for polarized M2 mononuclear phagocytes. Trends Immunol 23(11): 549-555.

Martinez, FO, Sica, A, Mantovani, A, Locati, M (2008) Macrophage activation and polarization. Front Biosci 13: 453-461.

Mathur, RK, Awasthi, A, Wadhone, P, Ramanamurthy, B, Saha, B (2004) Reciprocal CD40 signals through P38MAPK and ERK-1/2 induce counteracting immune responses. Nat Med 10(5): 540544.

McKee, AS, Pearce, EJ (2004) CD25+CD4+ Cells Contribute to Th2 Polarization during Helminth Infection by Suppressing Th1 Response Development. J Immunol 173(2): 1224-1231.

McManus, C, Berman, JW, Brett, FM, Staunton, H, Farrell, M, Brosnan, CF (1998) MCP-1, MCP-2 and MCP-3 expression in multiple sclerosis lesions: an immunohistochemical and in situ hybridization study. Journal of Neuroimmunology 86(1): 20-29.

Melendez, AJ (2008) Sphingosine kinase signalling in immune cells: potential as novel therapeutic targets. Biochim Biophys Acta 1784(1): 66-75.

Micallef, MJ, Yoshida, K, Kawai, S, Hanaya, T, Kohno, K, Arai, S, Tanimoto, T, Torigoe, K, Fujii, M, Ikeda, $M$, Kurimoto, $M$ (1997) In vivo antitumor effects of murine interferon-gamma-inducing factor/interleukin-18 in mice bearing syngeneic Meth A sarcoma malignant ascites. Cancer Immunol Immunother 43(6): 361-367.

Miles, SA, Conrad, SM, Alves, RG, Jeronimo, SM, Mosser, DM (2005) A role for IgG immune complexes during infection with the intracellular pathogen Leishmania. J Exp Med 201(5): 747754.

Mills, KH (2008) Induction, function and regulation of IL-17-producing T cells. Eur J Immunol 38(10): 2636-2649.

Modolell, M, Corraliza, IM, Link, F, Soler, G, Eichmann, K (1995) Reciprocal regulation of the nitric oxide synthase/arginase balance in mouse bone marrow-derived macrophages by TH1 and TH2 cytokines. Eur J Immunol 25(4): 1101-1104.

Mosser, D, Zhang, X (2008a) Interleukin-10: new perspectives on an old cytokine. Immunological Reviews 226: 205-218.

Mosser, DM (2003) The many faces of macrophage activation. J Leukoc Biol 73(2): 209-212.

Mosser, DM, Edwards, JP (2008b) Exploring the full spectrum of macrophage activation. Nature Reviews Immunology 8(12): 958-969. 
Mukherjee, S, Chen, LY, Papadimos, TJ, Huang, S, Zuraw, BL, Pan, ZK (2009) Lipopolysaccharidedriven Th2 cytokine production in macrophages is regulated by both MyD88 and TRAM. J Biol Chem 284(43): 29391-29398.

Munn, DH, Shafizadeh, E, Attwood, JT, Bondarev, I, Pashine, A, Mellor, AL (1999) Inhibition of T cell proliferation by macrophage tryptophan catabolism. J Exp Med 189(9): 1363-1372.

Murray, PJ (2006) Understanding and exploiting the endogenous interleukin-10/STAT3-mediated anti-inflammatory response. Curr Opin Pharmacol 6(4): 379-386.

Murugaiyan, G, Agrawal, R, Mishra, GC, Mitra, D, Saha, B (2006) Functional dichotomy in CD40 reciprocally regulates effector T cell functions. J Immunol 177(10): 6642-6649.

Nair, MG, Du, Y, Perrigoue, JG, Zaph, C, Taylor, JJ, Goldschmidt, M, Swain, GP, Yancopoulos, GD, Valenzuela, DM, Murphy, A, Karow, M, Stevens, S, Pearce, EJ, Artis, D (2009) Alternatively activated macrophage-derived RELM-\{alpha\} is a negative regulator of type 2 inflammation in the lung. J Exp Med 206(4): 937-952.

Nakamura, K, Kitani, A, Strober, W (2001) Cell contact-dependent immunosuppression by $\mathrm{CD} 4(+) \mathrm{CD} 25(+)$ regulatory $T$ cells is mediated by cell surface-bound transforming growth factor beta. J Exp Med 194(5): 629-644.

Nishimura, H, Nose, M, Hiai, H, Minato, N, Honjo, T (1999) Development of lupus-like autoimmune diseases by disruption of the PD-1 gene encoding an ITIM motif-carrying immunoreceptor. Immunity 11(2): 141-151.

Nishimura, H, Okazaki, T, Tanaka, Y, Nakatani, K, Hara, M, Matsumori, A, Sasayama, S, Mizoguchi, A, Hiai, H, Minato, N, Honjo, T (2001) Autoimmune dilated cardiomyopathy in PD-1 receptordeficient mice. Science 291(5502): 319-322.

Nolan, A, Kobayashi, H, Naveed, B, Kelly, A, Hoshino, Y, Hoshino, S, Karulf, MR, Rom, WN, Weiden, MD, Gold, JA (2009) Differential Role for CD80 and CD86 in the Regulation of the Innate Immune Response in Murine Polymicrobial Sepsis. PLoS ONE 4(8): e6600.

Nolan, A, Weiden, M, Kelly, A, Hoshino, Y, Hoshino, S, Mehta, N, Gold, JA (2008) CD40 and CD80/86 act synergistically to regulate inflammation and mortality in polymicrobial sepsis. Am J Respir Crit Care Med 177(3): 301-308.

Nurieva, RI, Duong, J, Kishikawa, H, Dianzani, U, Rojo, JM, Ho, I, Flavell, RA, Dong, C (2003) Transcriptional regulation of th2 differentiation by inducible costimulator. Immunity 18(6): 801811.

O'Connor, RA, Prendergast, CT, Sabatos, CA, Lau, CW, Leech, MD, Wraith, DC, Anderton, SM (2008) Cutting edge: Th1 cells facilitate the entry of Th17 cells to the central nervous system during experimental autoimmune encephalomyelitis. J Immunol 181(6): 3750-3754.

O'Keefe, GM, Nguyen, VT, Ping Tang, LL, Benveniste, EN (2001) IFN-gamma regulation of class II transactivator promoter IV in macrophages and microglia: involvement of the suppressors of cytokine signaling-1 protein. J Immunol 166(4): 2260-2269.

O'Shea, JJ, Murray, PJ (2008) Cytokine signaling modules in inflammatory responses. Immunity 28(4): 477-487. 
Okano, M, Satoskar, AR, Nishizaki, K, Abe, M, Harn, DA, Jr. (1999) Induction of Th2 Responses and IgE Is Largely Due to Carbohydrates Functioning as Adjuvants on Schistosoma mansoni Egg Antigens. J Immunol 163(12): 6712-6717.

Okuda, Y, Sakoda, S, Bernard, CC, Fujimura, H, Saeki, Y, Kishimoto, T, Yanagihara, T (1998) IL-6deficient mice are resistant to the induction of experimental autoimmune encephalomyelitis provoked by myelin oligodendrocyte glycoprotein. Int Immunol 10(5): 703-708.

Oppmann, B, Lesley, R, Blom, B, Timans, JC, Xu, Y, Hunte, B, Vega, F, Yu, N, Wang, J, Singh, K, Zonin, F, Vaisberg, E, Churakova, T, Liu, M, Gorman, D, Wagner, J, Zurawski, S, Liu, Y, Abrams, JS, Moore, KW, Rennick, D, de Waal-Malefyt, R, Hannum, C, Bazan, JF, Kastelein, RA (2000) Novel p19 protein engages IL-12p40 to form a cytokine, IL-23, with biological activities similar as well as distinct from IL-12. Immunity 13(5): 715-725.

Ouma, JH, Vennervald, BJ, Butterworth, AE (2001) Morbidity in schistosomiasis: an update. Trends Parasitol 17(3): 117-118.

Ouyang, W, Ranganath, SH, Weindel, K, Bhattacharya, D, Murphy, TL, Sha, WC, Murphy, KM (1998) Inhibition of Th1 development mediated by GATA-3 through an IL-4-independent mechanism. Immunity 9(5): 745-755.

Ozinsky, A, Smith, KD, Hume, D, Underhill, DM (2000) Co-operative induction of pro-inflammatory signaling by Toll-like receptors. J Endotoxin Res 6(5): 393-396.

Pandiyan, P, Zheng, L, Ishihara, S, Reed, J, Lenardo, MJ (2007) CD4+CD25+Foxp3+ regulatory T cells induce cytokine deprivation-mediated apoptosis of effector CD4+ T cells. Nat Immunol 8(12): 1353-1362.

Parronchi, P, De Carli, M, Manetti, R, Simonelli, C, Sampognaro, S, Piccinni, MP, Macchia, D, Maggi, E, Del Prete, G, Romagnani, S (1992) IL-4 and IFN (alpha and gamma) exert opposite regulatory effects on the development of cytolytic potential by Th1 or Th2 human T cell clones. $J$ Immunol 149(9): 2977-2983.

Pearce, EJ, C, MK, Sun, J, J, JT, McKee, AS, Cervi, L (2004) Th2 response polarization during infection with the helminth parasite Schistosoma mansoni. Immunol Rev 201: 117-126.

Pearce, EJ, Caspar, P, Grzych, JM, Lewis, FA, Sher, A (1991) Downregulation of Th1 cytokine production accompanies induction of Th2 responses by a parasitic helminth, Schistosoma mansoni. J Exp Med 173(1): 159-166.

Perlmutter, LS, Scott, SA, Barron, E, Chui, HC (1992) MHC class II-positive microglia in human brain: association with Alzheimer lesions. J Neurosci Res 33(4): 549-558.

Perona-Wright, G, Jenkins, SJ, O'Connor, RA, Zienkiewicz, D, McSorley, HJ, Maizels, RM, Anderton, SM, MacDonald, AS (2009) A Pivotal Role for CD40-Mediated IL-6 Production by Dendritic Cells during IL-17 Induction In Vivo. J Immunol 182(5): 2808-2815.

Pesce, JT, Ramalingam, TR, Mentink-Kane, MM, Wilson, MS, El Kasmi, KC, Smith, AM, Thompson, RW, Cheever, AW, Murray, PJ, Wynn, TA (2009a) Arginase-1-expressing macrophages suppress Th2 cytokine-driven inflammation and fibrosis. PLoS Pathog 5(4): e1000371. 
Pesce, JT, Ramalingam, TR, Wilson, MS, Mentink-Kane, MM, Thompson, RW, Cheever, AW, Urban, JF, Jr., Wynn, TA (2009b) Retnla (relmalpha/fizz1) suppresses helminth-induced Th2-type immunity. PLoS Pathog 5(4): e1000393.

Pluddemann, A, Neyen, C, Gordon, S (2007) Macrophage scavenger receptors and host-derived ligands. Methods 43(3): 207-217.

Poudrier, J, van Essen, D, Morales-Alcelay, S, Leanderson, T, Bergthorsdottir, S, Gray, D (1998) CD40 ligand signals optimize $T$ helper cell cytokine production: role in Th2 development and induction of germinal centers. Eur J Immunol 28(10): 3371-3383.

Pouliot, P, Camateros, P, Radzioch, D, Lambrecht, BN, Olivier, M (2009) Protein tyrosine phosphatases regulate asthma development in a murine asthma model. J Immunol 182(3): 13341340.

Qin, H, Wilson, CA, Lee, SJ, Zhao, X, Benveniste, EN (2005) LPS induces CD40 gene expression through the activation of NF-kappaB and STAT-1alpha in macrophages and microglia. Blood 106(9): 3114-3122.

Qin, H, Wilson, CA, Roberts, KL, Baker, BJ, Zhao, X, Benveniste, EN (2006) IL-10 Inhibits Lipopolysaccharide-Induced CD40 Gene Expression through Induction of Suppressor of Cytokine Signaling-3. J Immunol 177(11): 7761-7771.

Raes, G, De Baetselier, P, Noel, W, Beschin, A, Brombacher, F, Hassanzadeh Gh, G (2002) Differential expression of FIZZ1 and Ym1 in alternatively versus classically activated macrophages. J Leukoc Biol 71(4): 597-602.

Rahim, SS, Khan, N, Boddupalli, CS, Hasnain, SE, Mukhopadhyay, S (2005) Interleukin-10 (IL-10) mediated suppression of IL-12 production in RAW 264.7 cells also involves c-rel transcription factor. Immunology 114(3): 313-321.

Ramalingam, TR, Pesce, JT, Mentink-Kane, MM, Madala, S, Cheever, AW, Comeau, MR, Ziegler, SF, Wynn, TA (2009) Regulation of helminth-induced Th2 responses by thymic stromal lymphopoietin. J Immunol 182(10): 6452-6459.

Reis e Sousa, C, Hieny, S, Scharton-Kersten, T, Jankovic, D, Charest, H, Germain, RN, Sher, A (1997) In vivo microbial stimulation induces rapid CD40 ligand-independent production of interleukin 12 by dendritic cells and their redistribution to T cell areas. J Exp Med 186(11): 1819-1829.

Rezende, SA, Lambertucci, JR, Goes, AM (1997) Role of immune complexes from patients with different clinical forms of schistosomiasis in the modulation of in vitro granuloma research. Mem Inst Oswaldo Cruz 92(5): 683-687.

Rincon, M, Anguita, J, Nakamura, T, Fikrig, E, Flavell, RA (1997) Interleukin (IL)-6 directs the differentiation of IL-4-producing CD4+ T cells. J Exp Med 185(3): 461-469.

Robertson, JM, Jensen, PE, Evavold, BD (2000) DO11.10 and OT-II T cells recognize a C-terminal ovalbumin 323-339 epitope. J Immunol 164(9): 4706-4712.

Roers, A, Siewe, L, Strittmatter, E, Deckert, M, Schluter, D, Stenzel, W, Gruber, AD, Krieg, T, Rajewsky, K, Muller, W (2004) T cell-specific inactivation of the interleukin 10 gene in mice results in enhanced T cell responses but normal innate responses to lipopolysaccharide or skin irritation. $J$ Exp Med 200(10): 1289-1297. 
Roncarolo, MG, Gregori, S (2008) Is FOXP3 a bona fide marker for human regulatory T cells? Eur J Immunol 38(4): 925-927.

Rückerl, D, Heßmann, M, Yoshimoto, T, Ehlers, S, Hölscher, C (2006) Alternatively activated macrophages express the IL-27 receptor alpha chain WSX-1. Immunobiology 211(6-8): 427-436.

Russell, DG, Vanderven, BC, Glennie, S, Mwandumba, H, Heyderman, RS (2009) The macrophage marches on its phagosome: dynamic assays of phagosome function. Nat Rev Immunol 9(8): 594600 .

Sakaguchi, S (2004) Naturally arising CD4+ regulatory t cells for immunologic self-tolerance and negative control of immune responses. Annu Rev Immunol 22: 531-562.

Sanjuan, MA, Dillon, CP, Tait, SW, Moshiach, S, Dorsey, F, Connell, S, Komatsu, M, Tanaka, K, Cleveland, JL, Withoff, S, Green, DR (2007) Toll-like receptor signalling in macrophages links the autophagy pathway to phagocytosis. Nature 450(7173): 1253-1257.

Santoro, F, Vandemeulebroucke, B, Capron, A (1979) Schistosoma mansoni: circulating antigens and immune complexes in infected mice. Exp Parasitol 47(3): 392-402.

Schebesch, C, Kodelja, V, Muller, C, Hakij, N, Bisson, S, Orfanos, CE, Goerdt, S (1997) Alternatively activated macrophages actively inhibit proliferation of peripheral blood lymphocytes and CD4+ $T$ cells in vitro. Immunology 92(4): 478-486.

Schoenberger, SP, Toes, RE, van der Voort, El, Offringa, R, Melief, CJ (1998) T-cell help for cytotoxic T lymphocytes is mediated by CD40-CD40L interactions. Nature 393(6684): 480-483.

Schottelius, AJ, Mayo, MW, Sartor, RB, Baldwin, AS, Jr. (1999) Interleukin-10 signaling blocks inhibitor of kappaB kinase activity and nuclear factor kappaB DNA binding. J Biol Chem 274(45): 31868-31874.

Schramm, G, Falcone, FH, Gronow, A, Haisch, K, Mamat, U, Doenhoff, MJ, Oliveira, G, Galle, J, Dahinden, CA, Haas, H (2003) Molecular characterization of an interleukin-4-inducing factor from Schistosoma mansoni eggs. J Biol Chem 278(20): 18384-18392.

Schulz, EG, Mariani, L, Radbruch, A, Hofer, T (2009) Sequential polarization and imprinting of type $1 \mathrm{~T}$ helper lymphocytes by interferon-gamma and interleukin-12. Immunity 30(5): 673-683.

Seder, RA, Gazzinelli, R, Sher, A, Paul, WE (1993) Interleukin 12 acts directly on CD4+ T cells to enhance priming for interferon gamma production and diminishes interleukin 4 inhibition of such priming. Proc Natl Acad Sci U S A 90(21): 10188-10192.

Seder, RA, Kelsall, BL, Jankovic, D (1996) Differential roles for IL-12 in the maintenance of immune responses in infectious versus autoimmune disease. J Immunol 157(7): 2745-2748.

Seder, RA, Paul, WE, Davis, MM, Fazekas de St Groth, B (1992) The presence of interleukin 4 during in vitro priming determines the lymphokine-producing potential of CD4+ T cells from $T$ cell receptor transgenic mice. J Exp Med 176(4): 1091-1098.

Sempowski, GD, Beckmann, MP, Derdak, S, Phipps, RP (1994) Subsets of murine lung fibroblasts express membrane-bound and soluble IL-4 receptors. Role of IL-4 in enhancing fibroblast proliferation and collagen synthesis. J Immunol 152(7): 3606-3614. 
Serada, S, Fujimoto, M, Mihara, M, Koike, N, Ohsugi, Y, Nomura, S, Yoshida, H, Nishikawa, T, Terabe, F, Ohkawara, T, Takahashi, T, Ripley, B, Kimura, A, Kishimoto, T, Naka, T (2008) IL-6 blockade inhibits the induction of myelin antigen-specific Th17 cells and Th1 cells in experimental autoimmune encephalomyelitis. Proc Natl Acad Sci U S A 105(26): 9041-9046.

Setoguchi, R, Hori, S, Takahashi, T, Sakaguchi, S (2005) Homeostatic maintenance of natural Foxp3(+) CD25(+) CD4(+) regulatory T cells by interleukin (IL)-2 and induction of autoimmune disease by IL-2 neutralization. J Exp Med 201(5): 723-735.

Sewell, D, Qing, Z, Reinke, E, Elliot, D, Weinstock, J, Sandor, M, Fabry, Z (2003) Immunomodulation of experimental autoimmune encephalomyelitis by helminth ova immunization. Int Immunol 15(1): 59-69.

Shaw, MK, Lorens, JB, Dhawan, A, DalCanto, R, Tse, HY, Tran, AB, Bonpane, C, Eswaran, SL, Brocke, S, Sarvetnick, N, Steinman, L, Nolan, GP, Fathman, CG (1997) Local delivery of interleukin 4 by retrovirus-transduced $T$ lymphocytes ameliorates experimental autoimmune encephalomyelitis. $J$ Exp Med 185(9): 1711-1714.

Sher, A, Coffman, RL (1992) Regulation of immunity to parasites by T cells and T cell-derived cytokines. Annu Rev Immunol 10: 385-409.

Shoemaker, J, Saraiva, M, O'Garra, A (2006) GATA-3 directly remodels the IL-10 locus independently of IL-4 in CD4+ T cells. J Immunol 176(6): 3470-3479.

Skapenko, A, Kalden, JR, Lipsky, PE, Schulze-Koops, H (2005) The IL-4 Receptor \{alpha\}-ChainBinding Cytokines, IL-4 and IL-13, Induce Forkhead Box P3-Expressing CD25+CD4+ Regulatory T Cells from CD25-CD4+ Precursors. J Immunol 175(9): 6107-6116.

Smith-Garvin, JE, Koretzky, GA, Jordan, MS (2009) T cell activation. Annu Rev Immunol 27: 591619.

Smith, KA (1988) Interleukin-2: inception, impact, and implications. Science 240(4856): 1169-1176.

Sornasse, T, Larenas, PV, Davis, KA, de Vries, JE, Yssel, H (1996) Differentiation and stability of T helper 1 and 2 cells derived from naive human neonatal CD4+ T cells, analyzed at the single-cell level. J Exp Med 184(2): 473-483.

Stein, M, Keshav, S, Harris, N, Gordon, S (1992) Interleukin 4 potently enhances murine macrophage mannose receptor activity: a marker of alternative immunologic macrophage activation. J Exp Med 176(1): 287-292.

Steinfelder, S, Andersen, JF, Cannons, JL, Feng, CG, Joshi, M, Dwyer, D, Caspar, P, Schwartzberg, PL, Sher, A, Jankovic, D (2009) The major component in schistosome eggs responsible for conditioning dendritic cells for Th2 polarization is a T2 ribonuclease (omega-1). J Exp Med 206(8): 1681-1690.

Stenger, S, Donhauser, N, Thuring, H, Rollinghoff, M, Bogdan, C (1996) Reactivation of latent leishmaniasis by inhibition of inducible nitric oxide synthase. J Exp Med 183(4): 1501-1514.

Steube, KG, Meyer, C, Drexler, HG (1999) Constitutive protein expression of monocyte chemotactic protein-1 (MCP-1) by myelomonocytic cell lines and regulation of the secretion by anti- and proinflammatory stimuli. Leuk Res 23(9): 843-849. 
Stockinger, B, Veldhoen, M (2007) Differentiation and function of Th17 T cells. Curr Opin Immunol 19(3): 281-286.

Stout, RD, Suttles, J (2004) Functional plasticity of macrophages: reversible adaptation to changing microenvironments. J Leukoc Biol 76(3): 509-513.

Stout, RD, Suttles, J (1996a) The many roles of CD40 in cell-mediated inflammatory responses. Immunol Today 17(10): 487-492.

Stout, RD, Suttles, J, Xu, J, Grewal, IS, Flavell, RA (1996b) Impaired T cell-mediated macrophage activation in CD40 ligand-deficient mice. J Immunol 156(1): 8-11.

Stuve, O, Youssef, S, Slavin, AJ, King, CL, Patarroyo, JC, Hirschberg, DL, Brickey, WJ, Soos, JM, Piskurich, JF, Chapman, HA, Zamvil, SS (2002) The role of the MHC class II transactivator in class II expression and antigen presentation by astrocytes and in susceptibility to central nervous system autoimmune disease. J Immunol 169(12): 6720-6732.

Sutterwala, FS, Noel, GJ, Clynes, R, Mosser, DM (1997) Selective suppression of interleukin-12 induction after macrophage receptor ligation. J Exp Med 185(11): 1977-1985.

Sutterwala, FS, Noel, GJ, Salgame, P, Mosser, DM (1998) Reversal of proinflammatory responses by ligating the macrophage Fcgamma receptor type I. J Exp Med 188(1): 217-222.

Swain, SL (1995) CD4 T cell development and cytokine polarization: an overview. J Leukoc Biol 57(5): 795-798.

Szabo, SJ, Kim, ST, Costa, GL, Zhang, X, Fathman, CG, Glimcher, LH (2000) A Novel Transcription Factor, T-bet, Directs Th1 Lineage Commitment. Cell 100(6): 655-669.

Tian, L, Noelle, RJ, Lawrence, DA (1995) Activated T cells enhance nitric oxide production by murine splenic macrophages through gp39 and LFA-1. Eur J Immunol 25(1): 306-309.

Tierney, JB, Kharkrang, M, La Flamme, AC (2009) Type Il-activated macrophages suppress the development of experimental autoimmune encephalomyelitis. Immunol Cell Biol 87(3): 235-240.

Tilg, H, Trehu, E, Atkins, MB, Dinarello, CA, Mier, JW (1994) Interleukin-6 (IL-6) as an antiinflammatory cytokine: induction of circulating IL-1 receptor antagonist and soluble tumor necrosis factor receptor p55. Blood 83(1): 113-118.

Toes, RE, Schoenberger, SP, van der Voort, El, Offringa, R, Melief, CJ (1998) CD40-CD40Ligand interactions and their role in cytotoxic $T$ lymphocyte priming and anti-tumor immunity. Semin Immunol 10(6): 443-448.

Tran, EH, Hoekstra, K, van Rooijen, N, Dijkstra, CD, Owens, T (1998) Immune Invasion of the Central Nervous System Parenchyma and Experimental Allergic Encephalomyelitis, But Not Leukocyte Extravasation from Blood, Are Prevented in Macrophage-Depleted Mice. J Immunol 161(7): 3767-3775.

Tseng, SY, Otsuji, M, Gorski, K, Huang, X, Slansky, JE, Pai, SI, Shalabi, A, Shin, T, Pardoll, DM, Tsuchiya, H (2001) B7-DC, a new dendritic cell molecule with potent costimulatory properties for T cells. J Exp Med 193(7): 839-846. 
Veldhoen, M, Hocking, RJ, Atkins, CJ, Locksley, RM, Stockinger, B (2006) TGFbeta in the context of an inflammatory cytokine milieu supports de novo differentiation of IL-17-producing $T$ cells. Immunity 24(2): 179-189.

Victor, VM, Minano, M, Guayerbas, N, Del Rio, M, Medina, S, De la Fuente, M (1998) Effects of endotoxic shock in several functions of murine peritoneal macrophages. Mol Cell Biochem 189(12): 25-31.

Vignali, DA, Collison, LW, Workman, CJ (2008) How regulatory T cells work. Nat Rev Immuno/ 8(7): 523-532.

Vila-del Sol, V, Diaz-Munoz, MD, Fresno, M (2007) Requirement of tumor necrosis factor alpha and nuclear factor-kappaB in the induction by IFN-gamma of inducible nitric oxide synthase in macrophages. J Leukoc Biol 81(1): 272-283.

Vyas, JM, Van der Veen, AG, Ploegh, HL (2008) The known unknowns of antigen processing and presentation. Nat Rev Immunol 8(8): 607-618.

Wan, YY, Flavell, RA (2009) How Diverse--CD4 Effector T Cells and their Functions. J Mol Cell Biol $1(1): 20-36$.

Wang, S, Bajorath, J, Flies, DB, Dong, H, Honjo, T, Chen, L (2003) Molecular modeling and functional mapping of B7-H1 and B7-DC uncouple costimulatory function from PD-1 interaction. $J$ Exp Med 197(9): 1083-1091.

Waterhouse, P, Penninger, JM, Timms, E, Wakeham, A, Shahinian, A, Lee, KP, Thompson, CB, Griesser, H, Mak, TW (1995) Lymphoproliferative disorders with early lethality in mice deficient in Ctla-4. Science 270(5238): 985-988.

Weber, MS, Prod'homme, T, Youssef, S, Dunn, SE, Rundle, CD, Lee, L, Patarroyo, JC, Stuve, O, Sobel, RA, Steinman, L, Zamvil, SS (2007) Type II monocytes modulate T cell-mediated central nervous system autoimmune disease. Nat Med 13(8): 935-943.

Wierenga, EA, Snoek, M, de Groot, C, Chretien, I, Bos, JD, Jansen, HM, Kapsenberg, ML (1990) Evidence for compartmentalization of functional subsets of CD4+ T lymphocytes in atopic patients. J Immunol 144(12): 4651-4656.

Wildin, RS, Ramsdell, F, Peake, J, Faravelli, F, Casanova, JL, Buist, N, Levy-Lahad, E, Mazzella, M, Goulet, O, Perroni, L, Bricarelli, FD, Byrne, G, McEuen, M, Proll, S, Appleby, M, Brunkow, ME (2001) X-linked neonatal diabetes mellitus, enteropathy and endocrinopathy syndrome is the human equivalent of mouse scurfy. Nat Genet 27(1): 18-20.

Windhagen, A, Newcombe, J, Dangond, F, Strand, C, Woodroofe, MN, Cuzner, ML, Hafler, DA (1995) Expression of costimulatory molecules B7-1 (CD80), B7-2 (CD86), and interleukin 12 cytokine in multiple sclerosis lesions. J Exp Med 182(6): 1985-1996.

Wu, W, Mosteller, RD, Broek, D (2004) Sphingosine Kinase Protects Lipopolysaccharide-Activated Macrophages from Apoptosis. Mol. Cell. Biol. 24(17): 7359-7369.

Wu, Y, Xu, J, Shinde, S, Grewal, I, Henderson, T, Flavell, RA, Liu, Y (1995) Rapid induction of a novel costimulatory activity on B cells by CD40 ligand. Curr Biol 5(11): 1303-1311. 
Wynn, TA, Cheever, AW, Williams, ME, Hieny, S, Caspar, P, Kuhn, R, Muller, W, Sher, A (1998) IL10 Regulates Liver Pathology in Acute Murine Schistosomiasis mansoni But Is Not Required for Immune Down-Modulation of Chronic Disease. J Immunol 160(9): 4473-4480.

Wynn, TA, Thompson, RW, Cheever, AW, Mentink-Kane, MM (2004) Immunopathogenesis of schistosomiasis. Immunol Rev 201: 156-167.

Yamazaki, T, Akiba, H, Iwai, H, Matsuda, H, Aoki, M, Tanno, Y, Shin, T, Tsuchiya, H, Pardoll, DM, Okumura, K, Azuma, M, Yagita, H (2002) Expression of programmed death 1 ligands by murine T cells and APC. J Immunol 169(10): 5538-5545.

Yang, J, Castle, BE, Hanidu, A, Stevens, L, Yu, Y, Li, X, Stearns, C, Papov, V, Rajotte, D, Li, J (2005) Sphingosine Kinase 1 Is a Negative Regulator of CD4+ Th1 Cells. J Immunol 175(10): 6580-6588.

Yasukawa, H, Ohishi, M, Mori, H, Murakami, M, Chinen, T, Aki, D, Hanada, T, Takeda, K, Akira, S, Hoshijima, M, Hirano, T, Chien, KR, Yoshimura, A (2003) IL-6 induces an anti-inflammatory response in the absence of SOCS3 in macrophages. Nat Immunol 4(6): 551-556.

Yen, D, Cheung, J, Scheerens, H, Poulet, F, McClanahan, T, McKenzie, B, Kleinschek, MA, Owyang, A, Mattson, J, Blumenschein, W, Murphy, E, Sathe, M, Cua, DJ, Kastelein, RA, Rennick, D (2006) IL23 is essential for T cell-mediated colitis and promotes inflammation via IL-17 and IL-6. J Clin Invest 116(5): 1310-1316.

Zaccone, P, Fehervari, Z, Jones, FM, Sidobre, S, Kronenberg, M, Dunne, DW, Cooke, A (2003) Schistosoma mansoni antigens modulate the activity of the innate immune response and prevent onset of type 1 diabetes. Eur J Immunol 33(5): 1439-1449.

Zhang, H, Podojil, JR, Chang, J, Luo, X, Miller, SD (2010) TGF-\{beta\}-Induced Myelin PeptideSpecific Regulatory $T$ Cells Mediate Antigen-Specific Suppression of Induction of Experimental Autoimmune Encephalomyelitis. J Immunol 184(12): 6629-6636.

Zhang, X, Edwards, JP, Mosser, DM (2006a) Dynamic and Transient Remodeling of the Macrophage IL-10 Promoter during Transcription. J Immunol 177(2): 1282-1288.

Zhang, X, Edwards, JP, Mosser, DM (2006b) Dynamic and transient remodeling of the macrophage IL-10 promoter during transcription. Journal of Immunology 177(2): 1282-1288.

Zhang, X, Mosser, DM (2008) Macrophage activation by endogenous danger signals. J Pathol 214(2): 161-178.

Zheng, X, Hu, X, Zhou, G, Lu, Z, Qiu, W, Bao, J, Dai, Y (2008) Soluble egg antigen from Schistosoma japonicum modulates the progression of chronic progressive experimental autoimmune encephalomyelitis via Th2-shift response. J Neuroimmunol 194(1-2): 107-114.

Zhu, B, Guleria, I, Khosroshahi, A, Chitnis, T, Imitola, J, Azuma, M, Yagita, H, Sayegh, MH, Khoury, SJ (2006) Differential role of programmed death-ligand 1 [corrected] and programmed deathligand 2 [corrected] in regulating the susceptibility and chronic progression of experimental autoimmune encephalomyelitis. J Immunol 176(6): 3480-3489.

Zhu, J, Paul, WE (2008) CD4 T cells: fates, functions, and faults. Blood 112(5): 1557-1569. 


\section{Acknowledgements}

l'd firstly like to acknowledge all the invaluable fantastic advice and support from my supervisor Anne La Flamme. Anne was a constant source of knowledge and help throughout this whole project, without whom, I could not have gotten to this point.

l'd like to sincerely thank Nathaniel, who was a huge support both emotionally and mentally. I'm grateful for all the advice, the extreme patience, and the constant supply of delicious food and caring provided. Couldnt have done it without you.

As with Nathaniel, my brother Mike, and parents 'mum and dad', put up with me at my most insane moments. l'd like to thank them for all their support, and for not completely disowning me when the hunger and tiredness transformed me into a monster. My brother especially was a fantastic support throughout what feels like many many years of study.

Another special mention must go to Sara, who I feel created a great environment to work in and im thankful for having worked together. l'd like to thank Sara for all the great suggestions and help, but most of all, for her friendship which is priceless.

I also thank Dave, Ariane, Kirsty, Bridget and Arun who provided me with invaluable help at different points in this project which I couldn't have done without.

I thank Marcus 'Macrophage Guru', for teaching me the ropes and for all the advice.

In addition, I thank Sarabeth and Jenni for their support and the supply of Up \& Go drinks.

And thankyou also to John Miller, who was $99.9 \%$ of the time the first early bird to arrive, and was a friendly face in the early mornings.

Thankyou also to Jess, Tash, Ryan, Degee, Bhagyashree, Jonnel, Vishy and the whole KK601 lab, and thanks also to Patricia for all the help expecially when things went wrong.

Thankyou to Franca Ronchese and lan Hermans for the donation of mice.

Thanks also goes to Ken and Sonya from Taylor Preston, for the generous donation of sheep blood and strong support of scientific research.

I thank my close friends Hollie, Dinese, Laura, Maletina and their lil wee ones who often reminded me of what's really important in life.

Thanks to Te Ropu Awhina. Many thanks to Victoria Universityof Wellington, and The Neurological Foundation of NZ for funding. 